\title{
Stereoselective $\mathbf{S}_{\mathbf{N}} \mathbf{2}$-Substitutions using Polyfunctional Lithium Arylcuprates Prepared by an Iodine-Copper Exchange
}

\author{
M. Isabel Calaza, Xiaoyin Yang, Darunee Soorukram, and Paul Knochel* \\ Department Chemie, Ludwig-Maximilians-Universität, Butenandtstrasse 5-13, 81377, \\ München (Germany). \\ Paul.Knochel@cup.uni-muenchen.de
}

\section{Supporting Information}

\section{General considerations}

Unless otherwise indicated, all reactions were carried out with magnetic stirring and, if air or moisture sensitive, in flame-dried glassware under argon. Syringes used to transfer reagents and solvent were purged with argon prior to use. Reactions were monitored by gas chromotography (GC and GC-MS) or thin layer chromotography (TLC). Enantiomeric purity was determined by chiral HPLC or capillary GC analysis. In all cases, the analysis was calibrated with a sample of the racemate.

Chiral HPLC:

column A: Chiralcel OD-H, $0.46 \mathrm{~cm}$ x $25 \mathrm{~cm}$

column B: Chiralcel OD, $0.46 \mathrm{~cm}$ x $25 \mathrm{~cm}$

column C: Chiralcel AD, $0.46 \mathrm{~cm}$ x $25 \mathrm{~cm}$

Chiral GC:

column A: TFA gamma-cyclodextrin, $30.0 \mathrm{~m}$ x $0.25 \mathrm{~mm}$

method A: $40^{\circ} \mathrm{C}(2 \mathrm{~min})$, ramp of $20^{\circ} \mathrm{C} / \mathrm{min}$ to $150{ }^{\circ} \mathrm{C}(45 \mathrm{~min})$

method B: $130{ }^{\circ} \mathrm{C}(100 \mathrm{~min})$.

method $\mathrm{C}: 150{ }^{\circ} \mathrm{C}(150 \mathrm{~min})$.

\section{Starting materials}

(R)-2-Iodo-2-cyclohexen-1-ol and (R)-2-iodo-2-cyclopenten-1-ol are literature known. ${ }^{[1]}$ and $(1 S, 2 Z)$-1-Butyl-2-butenyl-2,3,4,5,6-pentafluorobenzoate are literature known. ${ }^{[2]}$ 
Neophyllithium solutions were titrated using the method of Paquette. ${ }^{[3]}$

\section{Preparation of lithium dineophylcuprate $\left(\mathrm{Nphyl}_{2} \mathrm{CuLi}\right):^{[4]}$}

A dry and argon flushed $500 \mathrm{~mL}$ round-bottom flask was charged with lithium dust $(3.0 \mathrm{~g}$, $432 \mathrm{mmol})$ and 2,2-dimethyl-2-phenylethyl chloride $(14.0 \mathrm{~mL}, 86.9 \mathrm{mmol})$ in $n$-hexane $(75$ $\mathrm{mL}$ ). The reaction mixture was refluxed overnight. After cooling to $\mathrm{rt} n$-hexane was removed in vacuo and then dry diethyl ether was added. The resulting mixture was cannulated into a flame dried Schlenk tube and centrifuged (2000 rpm, $30 \mathrm{~min})$. The clear solution of neophyllithium thus obtained was titrated before use with menthol using $o$-phenanthroline as indicator and could be stored at $-30{ }^{\circ} \mathrm{C}$ for several days. Usaully $1.4 \mathrm{M} / \mathrm{Et}_{2} \mathrm{O}$ solution of the lithium reagent is obtained.

A dry and argon flushed $25 \mathrm{~mL}$ flask was charged with $\mathrm{CuCN}$ (110 mg, $1.2 \mathrm{mmol})$. Dry diethyl ether $(1 \mathrm{~mL})$ was added and the resulting suspension was cooled to $0{ }^{\circ} \mathrm{C}$. The freshly titrated solution of neophyllithium was then added slowly and the mixture was quickly warmed to rt and stirred for $10 \mathrm{~min}$, until a clear yellow solution of the desired cuprate was obtained.

\section{(R)-2-Iodo-2-cyclopenten-1-yl acetate (4a): ${ }^{[5]}$}

(R)-2-Iodo-2-cyclopenten-1-ol (1.6 g, $7.6 \mathrm{mmol})$ was dissolved in pyridine $(5.0 \mathrm{~mL})$ and $\mathrm{Ac}_{2} \mathrm{O}$ $(2.5 \mathrm{~mL})$. The resulting mixture was stirred for $12 \mathrm{~h}$ at $25^{\circ} \mathrm{C}$. It was diluted with $\mathrm{Et}_{2} \mathrm{O}(50 \mathrm{~mL})$ and washed with $2 \mathrm{M} \mathrm{HCl}(50 \mathrm{~mL})$ and brine $(50 \mathrm{~mL} \times 2)$. The organic phase was dried $\left(\mathrm{Na}_{2} \mathrm{SO}_{4}\right)$. The solvent was removed and the crude product purified by column chromatography $\left(\mathrm{SiO}_{2}\right.$, pentane/diethyl ether $\left.=6: 1\right)$ to give $1.54 \mathrm{~g}(80 \%)$ of $\mathbf{4 a}$ as a colorless oil.

GC (column A, method A): $t_{R} / \min =8.7$ (major), 13.0 (minor); $96.6 \%$ ee. $[\alpha]_{\mathrm{D}}^{20}+33.8^{\circ}\left(\mathrm{c} 1.16, \mathrm{CH}_{2} \mathrm{Cl}_{2}\right)$

${ }^{1} \mathrm{H}-\mathrm{NMR}\left(\mathrm{CDCl}_{3}, 300 \mathrm{MHz}\right): \delta=6.36(\mathrm{~m}, 1 \mathrm{H}), 5.63(\mathrm{~m}, 1 \mathrm{H}), 2.51-2.16(\mathrm{~m}, 3 \mathrm{H}), 2.03(\mathrm{~s}, 3 \mathrm{H})$, $1.72-1.86(\mathrm{~m}, 1 \mathrm{H})$.

${ }^{13} \mathrm{C}-\mathrm{NMR}\left(\mathrm{CDCl}_{3}, 75 \mathrm{MHz}\right): \delta=170.6,145.7,92.7,83.8,33.0,29.9,21.1$.

IR (film): 1738 (vs), 1372 (m), 1233 (vs), 1034 (s), 926 (m), 808 (w).

MS (EI, $70 \mathrm{ev),} \mathrm{m/z} \mathrm{( \% ):} 209$ (4, $\left.\mathrm{M}^{+}-\mathrm{CH}_{3} \mathrm{CO}\right), 192$ (21), 125 (48), 83 (100), 66 (35), 65 (36), 55 (6).

Anal. calcd. for $\mathrm{C}_{7} \mathrm{H}_{9} \mathrm{IO}_{2}$ (252.05): C 33.36, H 3.60; found: C 33.78, H 3.22.

(R)-2-Iodo-2-cyclohexen-1-yl ethyl acetate $(4 \mathrm{~b}):^{[5]}$ 
To a solution of $(R)$-2-iodo-cyclohexen-1-ol (3.7 $\mathrm{g}, 16.5 \mathrm{mmol})$ in $20 \mathrm{~mL}$ pyridine, acetic anhydride ( $8.5 \mathrm{~g}, 82.6 \mathrm{mmol})$ was added at $\mathrm{rt}$. The resulting mixture was stirred at $\mathrm{rt}$ for $12 \mathrm{~h}$ and then diluted with $100 \mathrm{~mL}$ diethyl ether. The solution was washed with $2 \mathrm{M} \mathrm{HCL}(100 \mathrm{~mL})$ and brine $(100 \mathrm{~mL} \times 2)$. The organic phase was dried $\left(\mathrm{NaSO}_{4}\right)$ and concentrated in vacuum. The residue was purified by column chromatography $\left(\mathrm{SiO}_{2}\right.$, pentane/diethyl ether $\left.=10: 1\right)$ to give $4.2 \mathrm{~g}(95 \%)$ of $\mathbf{4 b}$ as a colorless oil.

GC (column A, method A): $t_{R} / \min =10.24$ (major), 11.04 (minor); $98 \%$ ee.

$[\alpha]_{\mathrm{D}}^{20}+21.6\left(\mathrm{c} 1.08, \mathrm{CH}_{2} \mathrm{Cl}_{2}\right)$

${ }^{1} \mathrm{H}-\mathrm{NMR}\left(\mathrm{CDCl}_{3}, 300 \mathrm{MH}_{\mathrm{Z}}\right): \delta=6.65(\mathrm{~m} \mathrm{1H}), 539(\mathrm{~m}, 1 \mathrm{H}), 2.11(\mathrm{~s} .3 \mathrm{H}), 2.11-1.70(\mathrm{~m}, 6 \mathrm{H})$.

${ }^{13} \mathrm{C}-\mathrm{NMR}\left(\mathrm{CDCl}_{3}, 75 \mathrm{MH}_{\mathrm{Z}}\right): \delta=170.5,143.9,95.8,73.8,30.3,29.5,21.6,17.8$.

IR (film): 2944 (w), 1735 (vs), 1427 (w), 1371 (m), 1233 (vs), 977 (m), 917 (w), 730 (w).

MS (EI, $70 \mathrm{ev),} \mathrm{m/z} \mathrm{( \% ):} 206$ (4) [M-AcO-H] $]^{+}, 139$ (85), 126 (15), 97 (100), 79 (47), 55 (4).

Anal. calcd. for $\mathrm{C}_{8} \mathrm{H}_{11} \mathrm{IO}_{2}$ (252.05): C 36.11, H 4.17; found: C 33.38, H 4.06.

\section{1-d-2-Iodo-2-cyclohexenyl acetate (4c):}

To a solution of 2-iodo-2-cyclohex-1-one $(1.11 \mathrm{~g}, 5 \mathrm{mmol})$ and $\mathrm{CeCl}_{3} \cdot 7 \mathrm{H}_{2} \mathrm{O}(1.86 \mathrm{~g}, 5 \mathrm{mmol})$ in $\mathrm{MeOH}(8 \mathrm{~mL})$ cooled at $0{ }^{\circ} \mathrm{C}, \mathrm{NaBD}_{4}$ was added in small portions. The resulting mixture was stirred at $25{ }^{\circ} \mathrm{C}$ for $3 \mathrm{~h}$. The reaction mixture was poured into cold water and extracted with $\mathrm{Et}_{2} \mathrm{O}(3 \times 20 \mathrm{~mL})$. The combined organic phase was washed with brine and dried $\left(\mathrm{Na}_{2} \mathrm{SO}_{4}\right)$. The solvent was removed and the crude product was purified by column chromatography $\left(\mathrm{SiO}_{2}\right.$, pentane/diethyl ether $\left.=5: 1\right)$ to give $856 \mathrm{mg}$ (76\% yield) of 1-d-2iodo-2-cyclohexenol as a colorless oil.

To a solution of 1-d-2-iodo-2-cyclohexenol $(788 \mathrm{mg}, 3.51 \mathrm{mmol})$ in pyridine $(3.1 \mathrm{~mL}), \mathrm{Ac}_{2} \mathrm{O}$ $(1.9 \mathrm{~mL})$ was added. The resulting mixture was stirred at $25{ }^{\circ} \mathrm{C}$ for $2 \mathrm{~h}$. The reaction mixture was quenched with $2 \mathrm{M} \mathrm{HCl}(5 \mathrm{~mL})$ and extracted with $\mathrm{Et}_{2} \mathrm{O}(3 \times 20 \mathrm{~mL})$. The combined organic phase was washed with $\mathrm{H}_{2} \mathrm{O}$, saturated aqueous $\mathrm{NaHCO}_{3}$ solution, brine, and dried $\left(\mathrm{Na}_{2} \mathrm{SO}_{4}\right)$. The solvent was removed and the crude product was purified by column chromatography $\left(\mathrm{SiO}_{2}\right.$, pentane/diethyl ether $\left.=10: 1\right)$ to give $775 \mathrm{mg}(83 \%$ yield $)$ of $\mathbf{4 c}$ as a colorless oil.

${ }^{1} \mathrm{H}-\mathrm{NMR}\left(\mathrm{CDCl}_{3}, 300 \mathrm{MHz}\right): \delta=6.65-6.50(\mathrm{~m}, 1 \mathrm{H}), 2.08-1.64(\mathrm{~m}, 9 \mathrm{H})$.

${ }^{13} \mathrm{C}-\mathrm{NMR}\left(\mathrm{CDCl}_{3}, 75 \mathrm{MHz}\right): \delta=169.6,144.0,95.8,72.0,30.3,29.5,21.6,17.7$.

IR (film): 2944 (m), 1738 (s), 1369 (m), 1264 (m), 1240 (s), 1013 (m), 922 (m).

MS (EI, $70 \mathrm{ev),} \mathrm{m/z} \mathrm{( \% ):} 208$ (3), 207 (5), 140 (79), 127 (10), 98 (100), 80 (33).

HRMS (EI): calcd. for $\mathrm{C}_{6} \mathrm{H}_{7} \mathrm{DI}\left[\mathrm{M}^{+}\right.$- OAc]: 207.9732, found: 207.9733. 


\section{4-[(1R)-(2-Iodo-2-cyclopenten-1-yl)] phenyl methyl ether (5a):}

Typical procedure A: A dry and argon flushed $25 \mathrm{~mL}$ flask, equipped with a magnetic stirrer and a septum, was charged with a solution of $\mathrm{Nphyl}_{2} \mathrm{CuLi}$ (1.2 mmol, 1.2 equiv). A solution of ethyl 4-iodoanisole $(281 \mathrm{mg}, 1.2 \mathrm{mmol})$ in THF $(2 \mathrm{~mL})$ was added over the solution of $\mathrm{Nphyl}_{2} \mathrm{CuLi}$, and the mixture was stirred at $0{ }^{\circ} \mathrm{C}$ until $\mathrm{I} / \mathrm{Cu}$-exchange was completed (30 min). The mixture was cooled at $-40{ }^{\circ} \mathrm{C}$ and a solution of $(R)$-2-iodo-2-cyclopenten-1-yl acetate (5a) (252 $\mathrm{mg}, 1.0 \mathrm{mmol}, 1.0$ equiv) in THF $(1.5 \mathrm{~mL})$ was added. The resulting reaction mixture was allowed to warm to $-20{ }^{\circ} \mathrm{C}$ and stirred at this temperature for $12 \mathrm{~h}$. Saturated aqueous $\mathrm{NH}_{4} \mathrm{Cl}$ sol. $(20 \mathrm{~mL})$ was added followed by $25 \%$ aqueous ammonia solution $(1 \mathrm{~mL})$. The reaction mixture was stirred at $25^{\circ} \mathrm{C}$ until the copper salts had dissolved and was extracted with $\mathrm{Et}_{2} \mathrm{O}$ (3 x $20 \mathrm{~mL})$. The combined extracts were washed with brine and dried $\left(\mathrm{Na}_{2} \mathrm{SO}_{4}\right)$. Evaporation of the solvents and purification by column chromatography $\left(\mathrm{SiO}_{2}\right.$, pentane, then pentane: $\mathrm{Et}_{2} \mathrm{O}=$ $30: 1)$ afforded $213 \mathrm{mg}$ (71 \% yield) of $\mathbf{5 a}$ as a colorless oil.

GC (column A, method $\mathrm{C}$ ): $t_{R} / \min =18.3$ (minor), 18.9 (major); $92.4 \%$ ee.

$[\alpha]_{\mathrm{D}}{ }^{20}-26.7^{\circ}\left(\mathrm{c} 1.19, \mathrm{CH}_{2} \mathrm{Cl}_{2}\right)$.

${ }^{1} \mathrm{H}-\mathrm{NMR}\left(\mathrm{CDCl}_{3}, 300 \mathrm{MHz}\right): \delta=7.20-7.04(\mathrm{~m}, 2 \mathrm{H}), 6.90-6.83(\mathrm{~m}, 2 \mathrm{H}), 6.30(\mathrm{~m}, 1 \mathrm{H}), 3.89-$ $3.80(\mathrm{~m}, 1 \mathrm{H}), 3.80(\mathrm{~s}, 3 \mathrm{H}), 2.58-2.33(\mathrm{~m}, 3 \mathrm{H}), 1.99-1.85(\mathrm{~m}, 1 \mathrm{H})$.

${ }^{13} \mathrm{C}-\mathrm{NMR}\left(\mathrm{CDCl}_{3}, 75 \mathrm{MHz}\right): \delta=158.4,140.8,136.1,128.7,113.9,100.9,58.7,55.2,33.9$, 33.0

IR (film): 2934 (m), 1611 (m), 1511 (vs), 1464 (m), 1303 (w), 1248 (vs), 1176 (s), 1037 (s), $828(\mathrm{~m}), 808(\mathrm{~m}), 543(\mathrm{w})$

MS (EI, $70 \mathrm{ev}), m / z(\%): 300\left(\mathrm{M}^{+}, 100\right), 269$ (4), 173 (42), 158 (29), 145 (15), 128 (16), 115 (13), 102 (9), 91 (4), 77 (4).

HRMS (EI): calcd. for $\mathrm{C}_{13} \mathrm{H}_{13} \mathrm{IO}\left[\mathrm{M}^{+}\right]$: 300.0011 , found: 300.0036 .

\section{Ethyl 4-[(1R)-(2-iodo-2-cyclopenten-1-yl)] benzoate (5b):}

The reaction was carried out according to typical procedure A with $\mathrm{Nphyl}_{2} \mathrm{CuLi}$ (1.2 mmol, 1.2 equiv), ethyl 4-iodobenzoate ( $0.331 \mathrm{~g}, 1.2 \mathrm{mmol}, 1.2 \mathrm{equiv})$ and $(R)$-2-iodo-2-cyclopenten-1-yl acetate (252 mg, $1.0 \mathrm{mmol}, 1.0$ equiv). Standard workup and purification by column chromatography $\left(\mathrm{SiO}_{2}, n\right.$-pentane/diethyl ether $\left.=30: 1\right)$ yielded $170 \mathrm{mg}(50 \%$ yield $)$ of $\mathbf{5 b}$ as a colorless oil.

GC (column A, method $\mathrm{C}$ ): $t_{R} / \min =81.5$ (minor), 86.4 (major); $97.0 \%$ ee. $[\alpha]_{\mathrm{D}}^{20}-14.4^{\circ}\left(\mathrm{c} 1.03, \mathrm{CH}_{2} \mathrm{Cl}_{2}\right)$. 
${ }^{1} \mathrm{H}-\mathrm{NMR}\left(\mathrm{CDCl}_{3}, 300 \mathrm{MHz}\right): \delta=7.96-7.90(\mathrm{~m}, 2 \mathrm{H}), 7.19-7.11(\mathrm{~m}, 2 \mathrm{H}), 6.29(\mathrm{~m}, 1 \mathrm{H}), 4.30(\mathrm{q}$, $J=7.1 \mathrm{~Hz}, 2 \mathrm{H}), 3.95-3.84(\mathrm{~m}, 1 \mathrm{H}), 2.55-2.28(\mathrm{~m}, 3 \mathrm{H}), 1.94-1.79(\mathrm{~m}, 1 \mathrm{H}), 1.31$ (t, $J=7.1 \mathrm{~Hz}$, $3 \mathrm{H})$.

${ }^{13} \mathrm{C}-\mathrm{NMR}\left(\mathrm{CDCl}_{3}, 75 \mathrm{MHz}\right): \delta=166.5,149.2,141.9,129.9,129.1,127.7,98.6,60.8,59.5$, 34.1, 33.0, 14.3 .

IR (film): 2935 (m), 1716 (vs), 1610 (m), 1418 (m), 1276 (vs), 1178 (m), 1102 (s), 1022 (m), $771(\mathrm{~m}), 707(\mathrm{~m}), 541(\mathrm{w})$.

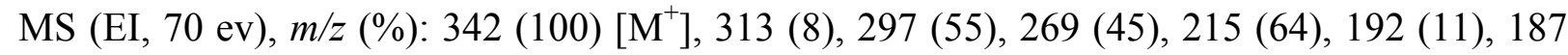

(9), 169 (10), 142 (50), 141 (55), 128 (24), 115 (32), 114 (11), 85 (6).

HRMS (EI): calcd. for $\mathrm{C}_{14} \mathrm{H}_{15} \mathrm{IO}_{2}\left[\mathrm{M}^{+}\right]$: 342.0117, found: 342.0154 .

\section{Ethyl 4-[(1S)-2-iodocyclohex-2-en-1-yl]beozoate (5c):}

The reaction was carried out according to typical procedure A with $\mathrm{Nphyl}_{2} \mathrm{CuLi}(1.2 \mathrm{mmol}$, 1.2 equiv), ethyl 4-iodobenzoate (331 mg, $1.2 \mathrm{mmol}, 1.2$ equiv) and (R)-2-iodo-cyclohex-2enyl ester (266 mg, $1.0 \mathrm{mmol}$ ). Standard workup and purification by column chromatography $\left(\mathrm{SiO}_{2}, n\right.$-pentane/diethyl ether $\left.=10: 1\right)$ yielded $274 \mathrm{mg}(77 \%$ yield $)$ of $\mathbf{5 c}$ as a colorless oil. HPLC (column $\mathrm{C}$, heptane $: i \mathrm{PrOH}=99: 1,0.5 \mathrm{ml} / \mathrm{min}$ ): $t_{R} / \mathrm{min}=18.3$ (minor), 19.8 (major); $98 \%$ ee.

$[\alpha]_{\mathrm{D}}^{20}+12.1\left(\mathrm{c} 0.99, \mathrm{CH}_{2} \mathrm{Cl}_{2}\right)$.

${ }^{1} \mathrm{H}-\mathrm{NMR}\left(\mathrm{CDCl}_{3}, 300 \mathrm{MH}_{\mathrm{Z}}\right): \delta=7.94(\mathrm{~d}, J=8.4 \mathrm{~Hz}, 2 \mathrm{H}), 7.20(\mathrm{~d}, J=8.4 \mathrm{~Hz}, 2 \mathrm{H}), 6.62(\mathrm{~m}$, $1 \mathrm{H}), 4.30$ (q, $J=7.1 \mathrm{~Hz}, 2 \mathrm{H}), 3.68(\mathrm{~m}, 1 \mathrm{H}), 2.09-1.53(\mathrm{~m}, 6 \mathrm{H}), 1.32$ (t, $J=7.1 \mathrm{~Hz}, 3 \mathrm{H})$.

${ }^{13} \mathrm{C}-\mathrm{NMR}\left(\mathrm{CDCl}_{3}, 75 \mathrm{MH}_{\mathrm{Z}}\right): \delta=149.8,141.2,130.1,129.4,128.7,100.2,61.2,53.0,33.9$, $29.7,18.1,14.8$.

IR (film): 3415 (w), 2937 (s), 1715 (vs), 1609 (m), 1444 (m), 1417 (m), 1366 (m), 1276 (vs), 1178 (s), 1102 (vs), 1021 (m), 769 (m), 707 (m).

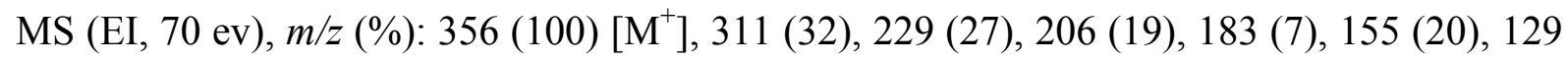
(25), 115 (13), 91 (7).

HRMS (EI): calcd. For $\mathrm{C}_{15} \mathrm{H}_{17} \mathrm{IO}_{2}\left[\mathrm{M}^{+}\right]$: 356.0273, found 356.0280 .

\section{4-[(1R)-2-iodocyclohex-2-en-1-yl]benzonitrile (5d):}

The reaction was carried out according to typical procedure A with $\mathrm{Nphyl}_{2} \mathrm{CuLi}(1.2 \mathrm{mmol}$, 1.2 equiv), 4-bromobenzonitrile (218 mg, $1.2 \mathrm{mmol}, 1.2$ equiv), and acetic acid ( $R$ )-2-iodocyclohex-2-enyl ester (266 mg, $1.0 \mathrm{mmol}, 1.0$ equiv). The temperature for the $\mathrm{Br} / \mathrm{Cu}$-exchange 
is rt. Standard workup and purification by column chromatography $\left(\mathrm{SiO}_{2}, n\right.$-pentane/diethyl ether $=15: 1)$ yielded $204 \mathrm{mg}(66 \%$ yield $)$ of $\mathbf{5 d}$ as a colorless oil.

GC (column A, method B): $t_{R} / \min =33.73$ (minor), 34.98 (major); $97 \%$ ee.

$[\alpha]_{\mathrm{D}}^{20}+18.5\left(\mathrm{c} 1.26, \mathrm{CH}_{2} \mathrm{Cl}_{2}\right)$.

${ }^{1} \mathrm{H}-\mathrm{NMR}\left(\mathrm{CDCl}_{3}, 300 \mathrm{MH}_{\mathrm{Z}}\right): \delta=7.56(\mathrm{~d}, J=8.4 \mathrm{~Hz}, 2 \mathrm{H}), 7.24(\mathrm{~d}, J=8.4 \mathrm{~Hz}, 2 \mathrm{H}), 6.63(\mathrm{~m}$, $1 \mathrm{H}), 3.69(\mathrm{~m}, 1 \mathrm{H}), 2.09-1.53(\mathrm{~m}, 6 \mathrm{H})$.

${ }^{13} \mathrm{C}-\mathrm{NMR}\left(\mathrm{CDCl}_{3}, 75 \mathrm{MH}_{\mathrm{Z}}\right): \delta=148.7,140.4,131.2,128.1,118.0,109.6,51.6,32.4,28.2$, 16.6 .

IR (film): 4306 (w), 2907 (m), 2229 (vs), 1604 (s), 1503 (s), 1411 (s), 975(s), 833 (vs), 690 (s), $563(\mathrm{vs}), 483(\mathrm{w})$.

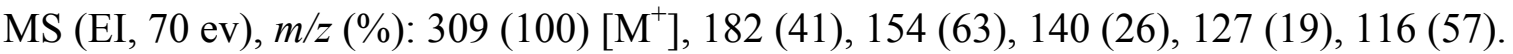

HRMS (EI): calcd. For $\mathrm{C}_{13} \mathrm{H}_{12} \mathrm{IN}\left[\mathrm{M}^{+}\right]$: 309.0014, found 309.0008 .

\section{1-[(1R)-2-Iodocyclohex-2-en-1-yl]-4-(trifluoromethyl)benzene (5e):}

The reaction was carried out according to typical procedure A with $\mathrm{Nphyl}_{2} \mathrm{CuLi}(1.2 \mathrm{mmol}$, 1.2 equiv), 1-iodo-4-trifluoromethylbenzene (326 mg, $1.2 \mathrm{mmol}, 1.2$ equiv), and acetic acid (R)-2-iodo-cyclohex-2-enyl ester (266 mg, $1.0 \mathrm{mmol}, 1.0$ equiv). Standard workup and purification by column chromatography $\left(\mathrm{SiO}_{2}, n\right.$-pentane/diethyl ether $\left.=15: 1\right)$ yielded 250 $\mathrm{mg}$ (70 \% yield) of $\mathbf{5 e}$ as a colorless oil.

GC (column A, method B): $t_{R} / \min =24.56$ (minor), 25.91 (major); $94 \%$ ee.

$[\alpha]_{\mathrm{D}}^{20}+11.7\left(\mathrm{c} 1.36, \mathrm{CH}_{2} \mathrm{Cl}_{2}\right)$.

${ }^{1} \mathrm{H}-\mathrm{NMR}\left(\mathrm{CDCl}_{3}, 300 \mathrm{MH}_{\mathrm{Z}}\right): \delta=7.52(\mathrm{~d}, J=8.0 \mathrm{~Hz}, 2 \mathrm{H}), 7.24(\mathrm{~d}, J=8.0 \mathrm{~Hz}, 2 \mathrm{H}), 6.62(\mathrm{~m}$, $1 \mathrm{H}), 3.68(\mathrm{~m}, 1 \mathrm{H}), 1.53-2.12(\mathrm{~m}, 6 \mathrm{H})$.

${ }^{13} \mathrm{C}-\mathrm{NMR}\left(\mathrm{CDCl}_{3}, 75 \mathrm{MH}_{\mathrm{Z}}\right): \delta=148.5,141.4,129.5,129.0,125.7,124\left(\mathrm{q}, J=271 \mathrm{H}_{\mathrm{Z}}\right), 99.8$, 73.3, 52.8, 33.9, 29.617 .9 .

IR (film): 2938 (s), 1618 (s), 1445 (m), 1418 (m), 1325 (vs), 1163 (vs), 1124 (vs), 1110 (vs), $985(\mathrm{~m}), 834(\mathrm{~m}), 606(\mathrm{w})$.

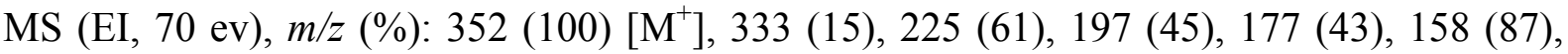
$128(20)$.

HRMS (EI): calcd. For $\mathrm{C}_{13} \mathrm{H}_{12} \mathrm{~F}_{3} \mathrm{I}\left[\mathrm{M}^{+}\right]$: 351.9936, found 351.9916.

\section{1-Iodo-4-[(1R)-2-iodocyclohex-2-en-1-yl]benzene (5f):}

The reaction was carried out according to typical procedure A with $\mathrm{Nphyl}_{2} \mathrm{CuLi}(1.2 \mathrm{mmol}$, 1.2 equiv), 1,4-diiodobenzene (394 mg, $1.2 \mathrm{mmol}, 1.2$ equiv), and acetic acid ( $R$ )-2-iodo- 
cyclohex-2-enyl ester (266 mg, $1.0 \mathrm{mmol}$ ). Standard workup and purification by column chromatography $\left(\mathrm{SiO}_{2}, n\right.$-pentane/diethyl ether $\left.=15: 1\right)$ yielded $336 \mathrm{mg}(82 \%$ yield $)$ of $\mathbf{5 f}$ as a colorless oil.

GC (column A, method $\mathrm{C}$ ): $t_{R} / \min =78.77$ (minor), 82.21 (major); $98 \%$ ee.

$[\alpha]_{\mathrm{D}}^{20}+25.2\left(\mathrm{c} 1.58, \mathrm{CH}_{2} \mathrm{Cl}_{2}\right)$

${ }^{1} \mathrm{H}-\mathrm{NMR}\left(\mathrm{CDCl}_{3}, 300 \mathrm{MH}_{\mathrm{Z}}\right): \delta=7.58(\mathrm{~d}, J=8.4 \mathrm{~Hz}, 2 \mathrm{H}), 6.88(\mathrm{~d}, J=8.4 \mathrm{~Hz}, 2 \mathrm{H}), 6.58(\mathrm{~m}$, $1 \mathrm{H}), 3.57(\mathrm{~m}, 1 \mathrm{H}), 2.14-1.46(\mathrm{~m}, 6 \mathrm{H})$.

${ }^{13} \mathrm{C}-\mathrm{NMR}\left(\mathrm{CDCl}_{3}, 75 \mathrm{MH}_{\mathrm{Z}}\right): \delta=142.5,139.4,136.1,129.1,98.8,90.7,50.9,32.2,28.0,16.3$.

IR (film): 2934 (s), 1627 (w), 1479 (s), 1400 (m), 1141 (w), 1060 (m), 1006 (s), 981 (m), 818 (s), $551(\mathrm{w})$.

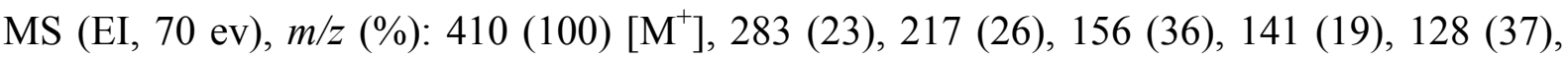
115 (17).

HRMS (EI): calcd. For $\mathrm{C}_{12} \mathrm{H}_{12} \mathrm{I}_{2}\left[\mathrm{M}^{+}\right]$: 409.9028, found 409.9017.

\section{1-Bromo-4-[(1R)-2-iodocyclohex-2-en-1-yl]benzene (5g):}

The reaction was carried out according to typical procedure A with $\mathrm{Nphyl}_{2} \mathrm{CuLi}(1.2 \mathrm{mmol}$, 1.2 equiv), 4-bromo-iodobenzene (339 mg, 1.2mmol, 1.2 equiv), and acetic acid ( $R$ )-2-iodocyclohex-2-enyl ester (266 mg, $1.0 \mathrm{mmol}$ ). Standard workup and purification by column chromatography ( $\mathrm{SiO}_{2}, n$-pentane) yielded $323 \mathrm{mg}$ (89\% yield) of $\mathbf{5 g}$ as a colorless oil.

$\mathrm{CC}$ (column A, method B): $t_{R} / \min =117.23$ (minor), 132.05 (major); 96\% ee.

$[\alpha]_{\mathrm{D}}^{20}+14.8\left(\mathrm{c} 1.43, \mathrm{CH}_{2} \mathrm{Cl}_{2}\right)$

${ }^{1} \mathrm{H}-\mathrm{NMR}\left(\mathrm{CDCl}_{3}, 300 \mathrm{MH}_{\mathrm{Z}}\right): \delta=7.38(\mathrm{~d}, J=8.4 \mathrm{~Hz}, 2 \mathrm{H}), 7.01(\mathrm{~d}, J=8.4 \mathrm{~Hz}, 2 \mathrm{H}), 6.58(\mathrm{~m}$, $1 \mathrm{H}), 3.61(\mathrm{~m}, 1 \mathrm{H}), 1.50-2.14(\mathrm{~m}, 6 \mathrm{H})$.

${ }^{13} \mathrm{C}-\mathrm{NMR}\left(\mathrm{CDCl}_{3}, 75 \mathrm{MH}_{\mathrm{Z}}\right): \delta=143.6,141.1,131.9,130.5,120.9,100.6,52.5,33.9,29,7$, 18.0 .

IR (film): 2934 (vs), 1898 (w), 1628 (m), 1483 (vs), 1442 (s), 1404 (s9, 1073 (s), 1010 (vs), $984(\mathrm{~s}), 895$ (m), 820 (s),701 (m9, $552(\mathrm{w})$.

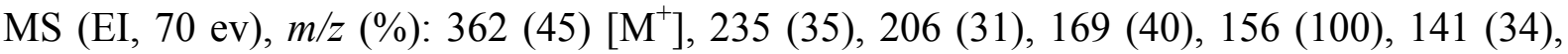
128 (77), 115 (29), 77 (15).

HRMS (EI): calcd. For $\mathrm{C}_{12} \mathrm{H}_{12} \mathrm{BrI}\left[\mathrm{M}^{+}\right]$: 361.9167, found 361.9153 .

\section{1-[(1R)-2-Iodocyclohex-2-en-1-yl]-4-methoxybenzene (5h):}

The reaction was carried out according to typical procedure A with $\mathrm{Nphyl}_{2} \mathrm{CuLi}(1.2 \mathrm{mmol}$, 1.2 equiv), 4-iodoanisole ( $291 \mathrm{mg}, 1.2 \mathrm{mmol}, 1.2$ equiv), and acetic acid (R)-2-iodo-cyclohex- 
2-enyl ester (266 mg, $1.0 \mathrm{mmol})$. Standard workup and purification by column chromatography $\left(\mathrm{SiO}_{2}, n\right.$-pentane/ $\left.\mathrm{Et}_{2} \mathrm{O}=150: 1\right)$ yielded $270 \mathrm{mg}(85 \%)$ of $\mathbf{5 h}$ as a colorless oil.

GC (column A, method B): $t_{R} / \min =96.5$ (minor), 102.2 (major); $95 \%$ ee.

$[\alpha]_{\mathrm{D}}^{20}+15.2\left(\mathrm{c} 1.04, \mathrm{CH}_{2} \mathrm{Cl}_{2}\right)$

${ }^{1} \mathrm{H}-\mathrm{NMR}\left(\mathrm{CDCl}_{3}, 300 \mathrm{MH}_{\mathrm{Z}}\right): \delta=7.04(\mathrm{~d}, J=8.4 \mathrm{~Hz}, 2 \mathrm{H}), 6.79(\mathrm{~d}, J=8.4 \mathrm{~Hz}, 2 \mathrm{H}), 6.56(\mathrm{~m}$, 1H), $3.73(\mathrm{~s}, 3 \mathrm{H}), 3.57(\mathrm{~m}, 1 \mathrm{H}), 2.12-1.54(\mathrm{~m}, 6 \mathrm{H})$.

${ }^{13} \mathrm{C}-\mathrm{NMR}\left(\mathrm{CDCl}_{3}, 75 \mathrm{MH}_{\mathrm{Z}}\right): \delta=158.7,140.4,136.7,129.7,114.1,102.5,55.6,52.2,34.1$, $29.7,18.2$.

IR (film): 2933 (vs), 2832 (s), 1610 (s), 1510 (vs), 1463 (s), 1302 (m), 1249 (vs), 1176 (s), $1036(\mathrm{~s}), 828(\mathrm{~m}), 602(\mathrm{w})$.

MS (EI, $70 \mathrm{ev),} \mathrm{m/z} \mathrm{( \% ):} 314$ (100) [M+1, 286 (4), 208 (18), 187 (31), 171(10), 159 (24), 144 (22), 121 (43), 108 (23), 77 (11).

HRMS (EI): calcd. For $\mathrm{C}_{13} \mathrm{H}_{15} \mathrm{IO}\left[\mathrm{M}^{+}\right]$: 314.0168 , found 314.0150 .

\section{1-\{4-[(1R)-2-Iodocyclohex-2-en-1-yl]phenyl\}ethanone (5i):}

The reaction was carried out according to typical procedure A with $\mathrm{Nphyl}_{2} \mathrm{CuLi}(1.2 \mathrm{mmol}$, 1.2 equiv), 4-iodo-acetobenzene (295 mg, $1.2 \mathrm{mmol}, 1.2$ equiv), and acetic acid ( $R$ )-2-iodocyclohex-2-enyl ester (266 mg, $1.0 \mathrm{mmol}$ ). Standard workup and purification by column chromatography $\left(\mathrm{SiO}_{2}, n\right.$-pentane/diethyl ether $\left.=10: 1\right)$ yielded $218 \mathrm{mg}(67 \%$ yield $)$ of $\mathbf{5 i}$ as a colorless oil.

HPLC (column $\mathrm{C}$, heptane $: i \mathrm{PrOH}=99: 1,0.4 \mathrm{ml} / \mathrm{min}$ ): $t_{R} / \mathrm{min}=47.19$ (minor), 51.78 (major); $89 \%$ ee.

$[\alpha]_{\mathrm{D}}^{20}+24.7\left(\mathrm{c} 1.43, \mathrm{CH}_{2} \mathrm{Cl}_{2}\right)$

${ }^{1} \mathrm{H}-\mathrm{NMR}\left(\mathrm{CDCl}_{3}, 300 \mathrm{MH}_{\mathrm{Z}}\right): \delta=7.87(\mathrm{~d}, J=8.4 \mathrm{~Hz}, 2 \mathrm{H}), 7.23(\mathrm{~d}, J=8.4 \mathrm{~Hz}, 2 \mathrm{H}), 6.63(\mathrm{~m}$, $1 \mathrm{H}), 3.69(\mathrm{~m}, 1 \mathrm{H}), 2.53(\mathrm{~s}, 3 \mathrm{H}), 2.12-1.57(\mathrm{~m}, 6 \mathrm{H})$.

${ }^{13} \mathrm{C}-\mathrm{NMR}\left(\mathrm{CDCl}_{3}, 75 \mathrm{MH}_{\mathrm{Z}}\right): \delta=198.1,150.1,141.3,136.1,129.0,100.0,53.0,33.9,29.7$, $27.0,18.1$.

IR (film): 2935 (s), 1681 (vs), 1605 (s), 1412 (m), 1357 (s), 1268 (s), 938 (m), 830 (m), 589 (m), $553(\mathrm{w})$.

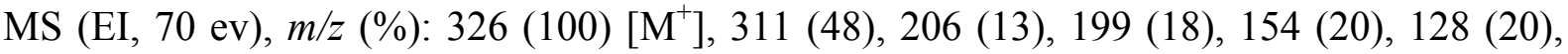
115 (14), 77 (7).

HRMS (EI): calcd. For $\mathrm{C}_{14} \mathrm{H}_{15} \mathrm{IO}\left[\mathrm{M}^{+}\right]$: 326.0168 , found 326.0162 . 


\section{1-[(1R)-2-Iodocyclohex-2-en-1-yl]-3-methoxybenzene (5j):}

The reaction was carried out according to typical procedure A with $\mathrm{Nphyl}_{2} \mathrm{CuLi}(1.2 \mathrm{mmol}$, 1.2 equiv), 3-iodoanisole (281 mg, $1.2 \mathrm{mmol}, 1.2$ equiv), and acetic acid ( $R$ )-2-iodo-cyclohex2-enyl ester (266 mg, $1.0 \mathrm{mmol})$. Standard workup and purification by column chromatography $\left(\mathrm{SiO}_{2}, n\right.$-pentane/diethyl ether $\left.=100: 1\right)$ yielded $270 \mathrm{mg}$ (86\% yield) of $\mathbf{5 j}$ as a colorless oil.

HPLC (column A, heptane : $i \operatorname{PrOH}=99: 1,0.2 \mathrm{ml} / \mathrm{min}$ ): $t_{R} / \mathrm{min}=31.52$ (minor), 38.05 (major); $92 \%$ ee.

$[\alpha]_{\mathrm{D}}^{20}+27.6\left(\mathrm{c} 1.27, \mathrm{CH}_{2} \mathrm{Cl}_{2}\right)$

${ }^{1} \mathrm{H}-\mathrm{NMR}\left(\mathrm{CDCl}_{3}, 300 \mathrm{MH}_{\mathrm{z}}\right): \delta=7.28(\mathrm{t}, J=8.4 \mathrm{~Hz}, 1 \mathrm{H}), 6.84-6.78(\mathrm{~m}, 3 \mathrm{H}), 6.68(\mathrm{~m}, 1 \mathrm{H})$, $3.84(\mathrm{~s}, 3 \mathrm{H}), 3.70(\mathrm{~m}, 1 \mathrm{H}), 2.17-1.63(\mathrm{~m}, 6 \mathrm{H})$.

${ }^{13} \mathrm{C}-\mathrm{NMR}\left(\mathrm{CDCl}_{3}, 75 \mathrm{MH}_{\mathrm{Z}}\right): \delta=160.0,146.2,140.7,129.7,121.2,114.8,112.0,101.4,55.6$, 53.0, 34.0, 29.1, 18.2.

IR (film): 2935 (vs), 2832 (w), 1660 (vs), 1583 (vs), 1464 (vs), 1485 (s), 1435 (s), 1347 (m), 1252 (vs), 1154 (s), 1052 (s), 985 (m), 779 (m), 700 (s), 568 (w).

MS (EI, $70 \mathrm{ev),} \mathrm{m/z} \mathrm{( \% ):} 314$ (100) [M+], 187 (46), 172 (9), 159 (14), 144 (12), 121 (37), 115 (30), 79 (15).

HRMS (EI): calcd. For $\mathrm{C}_{13} \mathrm{H}_{15} \mathrm{IO}\left[\mathrm{M}^{+}\right]$: 314.0168, found 314.0157 .

\section{Ethyl 4-(1-d-2-iodo-2-cyclohexen-1-yl)benzoate (5k)}

The reaction was carried out according to typical procedure A with $\mathrm{Nphyl}_{2} \mathrm{CuLi}(1.2 \mathrm{mmol}, 1.2$ equiv), ethyl 4-iodobenzoate (331 mg, $1.2 \mathrm{mmol}, 1.2$ equiv) and 1-d-2-iodo-2-cyclohexenyl acetate (4c) (267 mg, $1.0 \mathrm{mmol}, 1.0$ equiv) in THF (1.5 mL). Standard workup and purification by flash chromatography $\left(\mathrm{SiO}_{2}, n\right.$-pentane/diethyl ether $\left.=100: 1\right)$ yielded $189 \mathrm{mg}(53 \%$ yield $)$ of $5 \mathbf{k}$ as a colorless oil.

${ }^{1} \mathrm{H}-\mathrm{NMR}\left(\mathrm{CDCl}_{3}, 300 \mathrm{MHz}\right): \delta=7.97-7.96(\mathrm{~m}, 2 \mathrm{H}), 7.23-7.17(\mathrm{~m}, 2 \mathrm{H}), 6.65-6.60(\mathrm{~m}, 1 \mathrm{H})$, $4.30(\mathrm{q}, J=7.1 \mathrm{~Hz}, 2 \mathrm{H}), 2.18-2.04(\mathrm{~m}, 3 \mathrm{H}), 1.76-1.64(\mathrm{~m}, 1 \mathrm{H}), 1.62-1.50(\mathrm{~m}, 2 \mathrm{H}), 1.31(\mathrm{t}, J=$ $7.1 \mathrm{~Hz}, 3 \mathrm{H})$.

${ }^{13} \mathrm{C}-\mathrm{NMR}\left(\mathrm{CDCl}_{3}, 75 \mathrm{MHz}\right): \delta=167.0,149.7,141.2,130.1,129.4,128.7,100.2,61.2,52.6$, $33.9,29.7,18.1,14.8$.

IR (film): 2935 (m), 1716 (s), 1610 (m), 1276 (s), 1178 (m), 1104 (s), 1022 (m), 771 (m), 707 (m).

MS (EI, $70 \mathrm{ev),} \mathrm{m/z} \mathrm{( \% ):} 357\left[\mathrm{M}^{+}\right],(100), 312$ (37), 230 (23), 207 (31), 156 (28), 142 (15), 129 (48), $116(14)$. 
HRMS (EI): calcd.for $\mathrm{C}_{15} \mathrm{H}_{16} \mathrm{DIO}_{2}$ : 357.0335, found: 357.0334 .

\section{4-(2-Hex-1-ynylcyclohex-2-enyl)-benzoic acid ethyl ester (6a):}

A flame-dried $25 \mathrm{~mL}$ flask equipped with a magnetic stirring bar, an argon inlet, and a septum was charged with $\mathrm{PdCl}_{2}\left(\mathrm{PPh}_{3}\right)_{2}(35 \mathrm{mg}, 0.05 \mathrm{mmol})$, $\mathrm{CuI}(10 \mathrm{mg}, 0.05 \mathrm{mmol})$ and dry THF (2 $\mathrm{mL})$. A solution of ethyl $4-[(1 R)-(2$-iodocyclohex-2-en-1-yl)] benzoate (5c) (335 mg, 1.0 mmol, 95\% ee) in THF ( $2 \mathrm{~mL})$ was added dropwise. The reaction mixture was stirred at $25{ }^{\circ} \mathrm{C}$ for $5 \mathrm{~min}$, then dry $\mathrm{Et}_{3} \mathrm{~N}$ (304 mg, $3.0 \mathrm{mmol}$ ) and 1-hexyne $(90 \mathrm{mg}, 1.1 \mathrm{mmol})$ were added consecutively. The resulting yellow solution was stirred at $25{ }^{\circ} \mathrm{C}$ for $25 \mathrm{~h}$. The reaction mixture was quenched with saturated aqueous $\mathrm{NH}_{4} \mathrm{Cl}$ sol. $(20 \mathrm{~mL})$ and extracted with $\mathrm{Et}_{2} \mathrm{O}(3 \times 20 \mathrm{~mL})$. The combined extracts were washed with brine and dried $\left(\mathrm{Na}_{2} \mathrm{SO}_{4}\right)$. Evaporation of the solvents and purification by column chromatography $\left(\mathrm{SiO}_{2}\right.$, pentane/diethyl ether $\left.=80: 1\right)$ afforded 205 $\mathrm{mg}(70 \%$ yield $)$ of $\mathbf{6 a}$ as a colorless oil.

$[\alpha]_{\mathrm{D}}^{20}+1.43^{\circ}\left(\mathrm{c} 0.70, \mathrm{CH}_{2} \mathrm{Cl}_{2}\right)$

${ }^{1} \mathrm{H}-\mathrm{NMR}\left(\mathrm{CDCl}_{3}, 300 \mathrm{MHz}\right): \delta=7.92-7.86(\mathrm{~m}, 2 \mathrm{H}), 7.24-7.18(\mathrm{~m}, 2 \mathrm{H}), 6.22-6.17(\mathrm{~m}, 1 \mathrm{H})$, 4.30 (q, $J=7.1 \mathrm{~Hz}, 2 \mathrm{H}), 3.46-3.40(\mathrm{~m}, 1 \mathrm{H}), 2.16-2.08(\mathrm{~m}, 2 \mathrm{H}), 2.04-1.88$ (m, 3H), 1.64-1.40 (m, 4H), 1.36-1.24 (m, 2H), 1.22-1.10 (m, 2H), $1.10-0.96(\mathrm{~m}, 2 \mathrm{H}), 0.67(\mathrm{t}, J=7.2 \mathrm{~Hz}, 3 \mathrm{H})$.

${ }^{13} \mathrm{C}-\mathrm{NMR}\left(\mathrm{CDCl}_{3}, 75 \mathrm{MHz}\right): \delta=167.1,150.8,135.7,129.7,128.7,123.2,89.5,81.9,61.1$, $45.8,32.4,31.1,28.7,26.1,22.0,19.5,19.2,14.7,13.9$.

IR (film): 2933 (m), 1719 (s), 1610 (m), 1275 (s), 1178 (m), 1102 (s), 1022 (m).

MS (EI, $\left.70 \mathrm{ev),} \mathrm{m/z} \mathrm{( \% ):} 310\left[\mathrm{M}^{+}\right], 100\right), 295$ (7), 281 (23), 265 (44), 253 (19), 237 (42), 225 (12) 209 (12), 195 (43), 181 (51), 165 (72), 152 (33), 134 (28), 119 (29), 115 (29), 103 (35), 91 (41), 77 (17).

HRMS (EI): calcd. for $\mathrm{C}_{21} \mathrm{H}_{26} \mathrm{O}_{2}: 310.1933$, found: 310.1946 .

\section{Ethyl 4-[2-(4-(benzoyloxy)phenyl)-2-cyclohexen-1-yl]benzoate (6b):}

A flame-dried $25 \mathrm{~mL}$ flask equipped with a magnetic stirring bar, an argon inlet, and a septum was charged with benzoic acid 4-iodo phenyl ester $(243 \mathrm{mg}, 0.75 \mathrm{mmol})$ and dry THF (1 mL), and cooled to $-20{ }^{\circ} \mathrm{C}$. To the resulting solution, $i-\operatorname{PrMgCl}(1.45 \mathrm{M} / \mathrm{THF}, 0.8 \mathrm{~mL})$ was added dropwise. The resulting mixture was stirred at $-20{ }^{\circ} \mathrm{C}$ for $30 \mathrm{~min}$. The solution of $\mathrm{ZnBr}_{2}(1.5$ $\mathrm{M} / \mathrm{THF}, 1.5 \mathrm{~mL}$ ) was added dropwise, and the reaction mixture was stirred at $-20{ }^{\circ} \mathrm{C}$ for 15 min. Then the reaction mixture was warmed up to $25{ }^{\circ} \mathrm{C}$ for $30 \mathrm{~min}$. The resulting solution was cannulated to the flame-dried $25 \mathrm{~mL}$ flask which was charged with $\mathrm{Pd}(\mathrm{dba})_{2}(14 \mathrm{mg}, 25 \mathrm{mmol})$, dppf (14 g, $25 \mathrm{mmol})$, ethyl $4-[(1 R)-(2$-iodocyclohex-2-en-1-yl) benzoate (5c) (178 mg, 0.5 
mmol, $95 \%$ ee) and THF ( $3 \mathrm{~mL})$. The reaction mixture was stirred at $25{ }^{\circ} \mathrm{C}$ for $16 \mathrm{~h}$, quenched with saturated aqueous $\mathrm{NH}_{4} \mathrm{Cl}$ sol. $(20 \mathrm{~mL})$ and extracted with $\mathrm{Et}_{2} \mathrm{O}(3 \times 20 \mathrm{~mL})$. The combined extracts were washed with brine and dried $\left(\mathrm{Na}_{2} \mathrm{SO}_{4}\right)$. Evaporation of the solvents and purification by column chromatography $\left(\mathrm{SiO}_{2}\right.$, pentane/diethyl ether $\left.=10: 1\right)$ afforded $160 \mathrm{mg}$ (75\% yield) of $6 \mathbf{b}$ as a white solid, $\mathrm{mp}=128{ }^{\circ} \mathrm{C}$.

$[\alpha]_{\mathrm{D}}^{20}-95.0^{\circ}\left(\mathrm{c} 0.60, \mathrm{CH}_{2} \mathrm{Cl}_{2}\right)$

${ }^{1} \mathrm{H}-\mathrm{NMR}\left(\mathrm{CDCl}_{3}, 300 \mathrm{MHz}\right): \delta=8.10-8.04(\mathrm{~m}, 2 \mathrm{H}), 7.88-7.82(\mathrm{~m}, 2 \mathrm{H}), 7.58-7.50(\mathrm{~m}, 1 \mathrm{H})$, 7.44-7.36 (m, 2H), 7.24-7.18 (m, 4H), 6.98-6.92 (m, 2H), 6.38-6.32 (m, 1H), $4.26(\mathrm{q}, J=7.1$ $\mathrm{Hz}, 2 \mathrm{H}), 4.02$ - $3.94(\mathrm{~m}, 2 \mathrm{H}), 2.32-2.20(\mathrm{~m}, 2 \mathrm{H}), 2.12$ - $1.98(\mathrm{~m}, 1 \mathrm{H}), 1.82-1.70(\mathrm{~m}, 1 \mathrm{H}), 1.58-$ $1.42(\mathrm{~m}, 2 \mathrm{H}), 1.28(\mathrm{t}, J=7.1 \mathrm{~Hz}, 3 \mathrm{H})$.

${ }^{13} \mathrm{C}-\mathrm{NMR}\left(\mathrm{CDCl}_{3}, 75 \mathrm{MHz}\right): \delta=167.0,165.5,150.9,149.9,139.7,137.0,133.9,130.5,129.9$, $129.0,128.9,128.7,127.2,121.6,61.1,43.2,32.9,26.4,17.9,14.7$.

IR (film): 2934 (m), 1731 (s), 1720 (s), 1609 (m), 1506 (m), 1275 (s), 1205 (s), 1173 (s), 1102 (m), 1082 (m), 1065 (m), 1024 (m), 741 (s).

MS (EI, $70 \mathrm{ev}), m / z(\%): 426\left(5, \mathrm{M}^{+}\right), 115$ (2), $106(8), 105$ (100), 78 (2), 77 (19), 51 (2).

HRMS (EI): calcd. for $\mathrm{C}_{28} \mathrm{H}_{26} \mathrm{O}_{4}$ : 426.1831, found: 426.1812 .

anal. calcd. for $\mathrm{C}_{28} \mathrm{H}_{26} \mathrm{O}_{4}$ (426.18): C 78.85, H 6.14; found: C 78.59, H 6.25.

\section{Ethyl 4-[(1S)-2-butyl-2-cyclohexen-1-yl]benzoate (6c):}

To $\mathrm{Zn}$ foil $(690 \mathrm{mg}, 10 \mathrm{mmol})$ previously activated with 1,2-dibromoethane $(79 \mu \mathrm{L})$ and TMSCl $(49 \mu \mathrm{L})$ in THF $(1 \mathrm{~mL})$ was added a solution of iodobutane $(0.644 \mathrm{~g}, 3.5 \mathrm{mmol})$ in THF $(1.5 \mathrm{~mL})$. The reaction mixture was heated at $40{ }^{\circ} \mathrm{C}$ for $4 \mathrm{~h} . \mathrm{GC}$ analysis of hydrolyzed reaction aliquot showed the complete formation of the zinc reagent.

A flame-dried $25 \mathrm{~mL}$ flask was charged with $\mathrm{Pd}(\mathrm{dba})_{2}(14 \mathrm{mg}, 0.025 \mathrm{mmol})$, dppf (14 mg, $0.025 \mathrm{mmol})$ and THF $(1 \mathrm{~mL})$. Then the solution of ethyl 4 [(1R)-(2-iodocyclohex-2-en-1-yl) benzoate (5c) $(0.178 \mathrm{mg}, 0.5 \mathrm{mmol}, 95 \%$ ee) in THF $(2 \mathrm{~mL})$ was added dropwise followed by addition of freshly prepared butylzinc iodide $(1.4 \mathrm{M} / \mathrm{THF}, 1.5 \mathrm{~mL})$. The reaction mixture was refluxed at $80{ }^{\circ} \mathrm{C}$ for $12 \mathrm{~h}$. The reaction mixture was quenched with saturated aqueous $\mathrm{NH}_{4} \mathrm{Cl}$ sol. $(20 \mathrm{~mL})$ and extracted with $\mathrm{Et}_{2} \mathrm{O}(3 \mathrm{x} 20 \mathrm{~mL})$. The combined extracts were washed with brine and dried over $\mathrm{Na}_{2} \mathrm{SO}_{4}$. Evaporation of the solvents and purification by column chromatography $\left(\mathrm{SiO}_{2}\right.$, pentane/diethyl ether = $\left.100: 2\right)$ afforded $99 \mathrm{mg}(69 \%$ yield $)$ of $6 \mathbf{c}$ as a colorless oil. $[\alpha]_{\mathrm{D}}^{20}-74.08^{\circ}\left(\mathrm{c} 1.25, \mathrm{CH}_{2} \mathrm{Cl}_{2}\right)$. 
${ }^{1} \mathrm{H}-\mathrm{NMR}\left(\mathrm{CDCl}_{3}, 300 \mathrm{MHz}\right): \delta=7.92-7.86(\mathrm{~m}, 2 \mathrm{H}), 7.20-7.14(\mathrm{~m}, 2 \mathrm{H})$, 5.66-5.62 $(\mathrm{m}, 1 \mathrm{H})$, $4.29(\mathrm{q}, J=6.9 \mathrm{~Hz}, 2 \mathrm{H}), 3.36-3.28(\mathrm{~m}, 1 \mathrm{H}), 2.08-1.96(\mathrm{~m}, 2 \mathrm{H}), 1.94-1.82(\mathrm{~m}, 1 \mathrm{H}), 1.80-1.00$ $(\mathrm{m}, 12 \mathrm{H}), 0.74(\mathrm{t}, J=6.9 \mathrm{~Hz}, 3 \mathrm{H})$.

${ }^{13} \mathrm{C}-\mathrm{NMR}\left(\mathrm{CDCl}_{3}, 75 \mathrm{MHz}\right): \delta=165.7,150.2,137.0,128.4,127.5,127.2,123.0,59.7,43.1$, $34.6,31.7,28.9,24.4,21.4,17.8,13.4,13.0$.

IR (film): 2930 (m), 1720 (s), 1609 (m), 1276 (s), 1177 (m), 1102 (s), 1023 (m).

MS (EI, 70 ev), m/z (\%): 286 [M+'], (100), 241 (34), 229 (91), 216 (24), 215 (24), 201 (12), 171 (39), 157 (31), 141 (32), 129 (60), 115 (28), 91 (24), 77 (14).

HRMS (EI): calcd. for $\mathrm{C}_{19} \mathrm{H}_{26} \mathrm{O}_{2}: 286.1933$, found: 286.1915 .

\section{Ethyl 4-[(1R,2E)-1-methylhept-2-en-1-yl]benzoate (8):}

A dry and argon flushed $15 \mathrm{~mL}$ flask, equipped with a magnetic stirrer and a septum, was charged with a solution of $\mathrm{Nphyl}_{2} \mathrm{CuLi}$ (1.2 mmol, 1.2 equiv). Ethyl 4-iodobenzoate (331 mg, $1.2 \mathrm{mmol}, 1.2$ equiv) was added at $-78^{\circ} \mathrm{C}$. The resulting mixture was immediately warmed to $0{ }^{\circ} \mathrm{C}$ and kept stirring for $30 \mathrm{~min}$. Then the reaction was cooled to $-78{ }^{\circ} \mathrm{C}$ and the solution of $\mathrm{ZnBr}_{2}$ in THF $(1.5 \mathrm{M}, 0.8 \mathrm{~mL}, 1.2 \mathrm{mmol})$ was added at this temperature. After $10 \mathrm{~min}$, the solution of $7(320 \mathrm{mg}, 1.0 \mathrm{mmol})$ in THF $(1.5 \mathrm{~mL})$ was added at $-40{ }^{\circ} \mathrm{C}$. The mixture was allowed to warm to rt overnight. The reaction mixture was quenched with saturated aqueous $\mathrm{NH}_{4} \mathrm{Cl}$ solution and poured into water $(25 \mathrm{~mL})$. The aqueous phase was extracted with diethyl ether $(3 \times 30 \mathrm{~mL})$. The organic fractions were washed with brine, dried $\left(\mathrm{MgSO}_{4}\right)$ and concentrated in vacuo. Purification by flash chromatography $\left(\mathrm{SiO}_{2}, n\right.$-pentane/diethyl ether $=$ $80: 1)$ yielded $220 \mathrm{mg}$ ( $85 \%$ yield) of $\mathbf{8}$ as a colorless oil

HPLC (column B, heptane $: i \operatorname{PrOH}=99: 1,0.2 \mathrm{ml} / \mathrm{min}$ ): $t_{R} / \mathrm{min}=21.87$ (major), 23.86 (minor); 95\% ee.

$[\alpha]_{\mathrm{D}}{ }^{20}-35.4\left(\mathrm{c} 1.01, \mathrm{CH}_{2} \mathrm{Cl}_{2}\right)$

${ }^{1} \mathrm{H}-\mathrm{NMR}\left(\mathrm{CDCl}_{3}, 300 \mathrm{MH}_{\mathrm{Z}}\right): \delta=7.85(\mathrm{~d}, J=8.4 \mathrm{~Hz}, 2 \mathrm{H}), 7.19(\mathrm{~d}, J=8.4 \mathrm{~Hz}, 2 \mathrm{H}), 5.48-5.41$ $(\mathrm{m}, 2 \mathrm{H}), 4.29$ (q, $J=7.07 \mathrm{~Hz}, 2 \mathrm{H}), 3.40(\mathrm{~m}, 1 \mathrm{H}), 1.94(\mathrm{~m}, 2 \mathrm{H}), 1.33-1.22(\mathrm{~m} \mathrm{10H}), 0.80$ (t, $J$ $=7.07,3 \mathrm{H})$.

${ }^{13} \mathrm{C}-\mathrm{NMR}\left(\mathrm{CDCl}_{3}, 75 \mathrm{MH}_{\mathrm{Z}}\right): \delta=167.1,152.3,134.4,130.5,130.1,128.6,127.5,61.1,42.7$, $32.6,32.0,22.6,21.7,14.7,14.1$.

IR (film): 3409 (s), 2959 (s), 2932 (s), 1718 (vs), 1608 (w), 1465 (w), 1408 (w), 1367 (m), 1276 (vs), 1181 (m), 1107 (vs), 1019 (m), 856 (w), 771 (m), 707 (w).

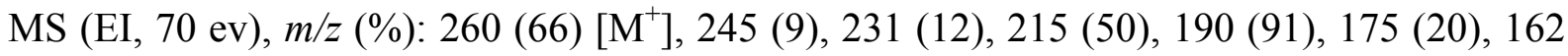
(41), 145 (66), 131 (100), 117 (41), 105 (12), 91 (15), 77 (7). 
HRMS (EI): calcd. For $\mathrm{C}_{17} \mathrm{H}_{24} \mathrm{O}_{2}\left[\mathrm{M}^{+}\right]$: 260.1776, found 260.1763. 
Appendix:

Determination of the enantiomer excess by chiral GC and chiral HPLC

\section{(R)-2-Iodo-2-cyclopenten-1-yl acetate(4a)}

$\mathrm{GC}\left(40{ }^{\circ} \mathrm{C}(2 \mathrm{~min})\right.$, ramp of $20^{\circ} \mathrm{C} / \mathrm{min}$ to $150{ }^{\circ} \mathrm{C}$ (45 min); TFA gamma-cyclodextrin, $30.0 \mathrm{~m}$ x $0.25 \mathrm{~mm}): t_{R} / \min 8.7(R), 9.6(S) ; 96.5 \%$ ee.

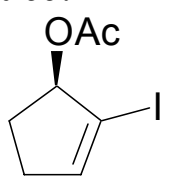

a) racemic

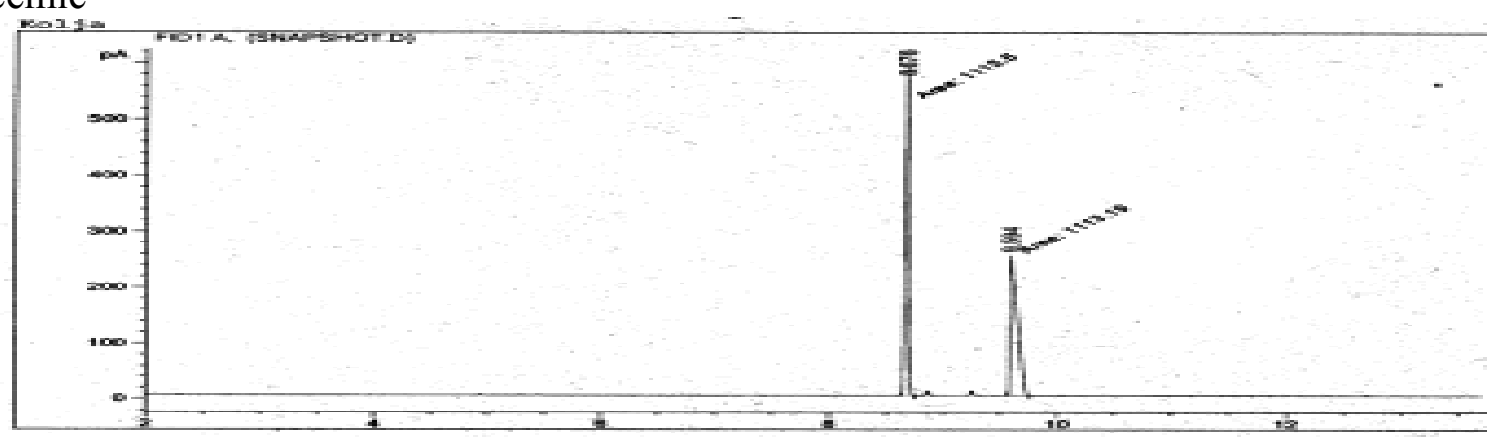

b)chiral

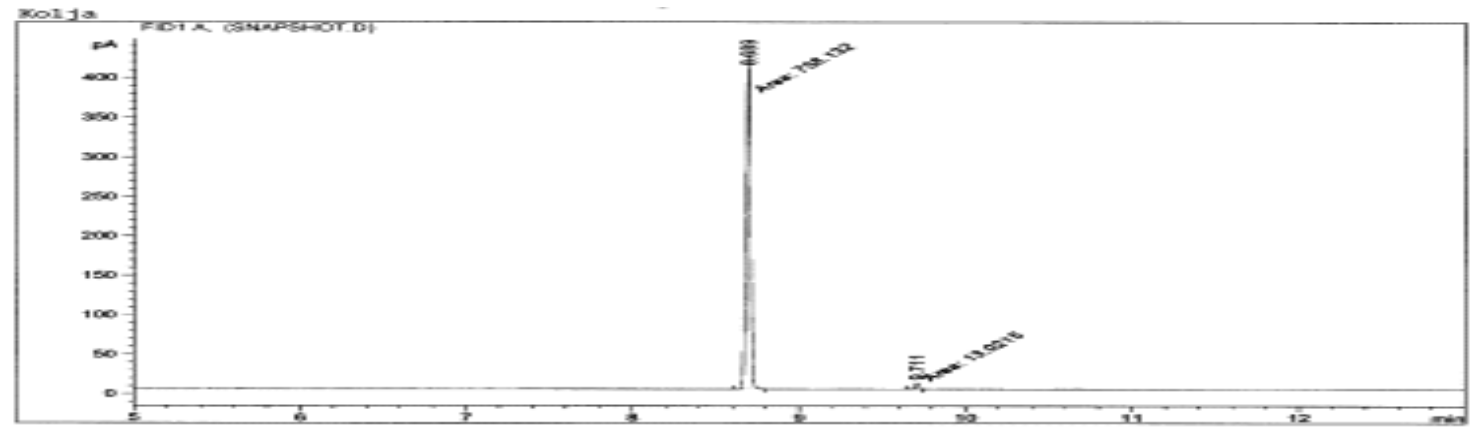

(R)-2-Iodo-2-cyclohexen-1-yl ethyl acetate (4b)

$\mathrm{GC}\left(40{ }^{\circ} \mathrm{C}(2 \mathrm{~min})\right.$, ramp of $20^{\circ} \mathrm{C} / \mathrm{min}$ to $150{ }^{\circ} \mathrm{C}$ (45 min); TFA gamma-cyclodextrin, $30.0 \mathrm{~m}$ x $0.25 \mathrm{~mm}): t_{R} / \min 8.7(R), 9.6(S) ; 98 \%$ ee.<smiles>CC(=O)OC1CCCC=C1I</smiles>

a): racemic

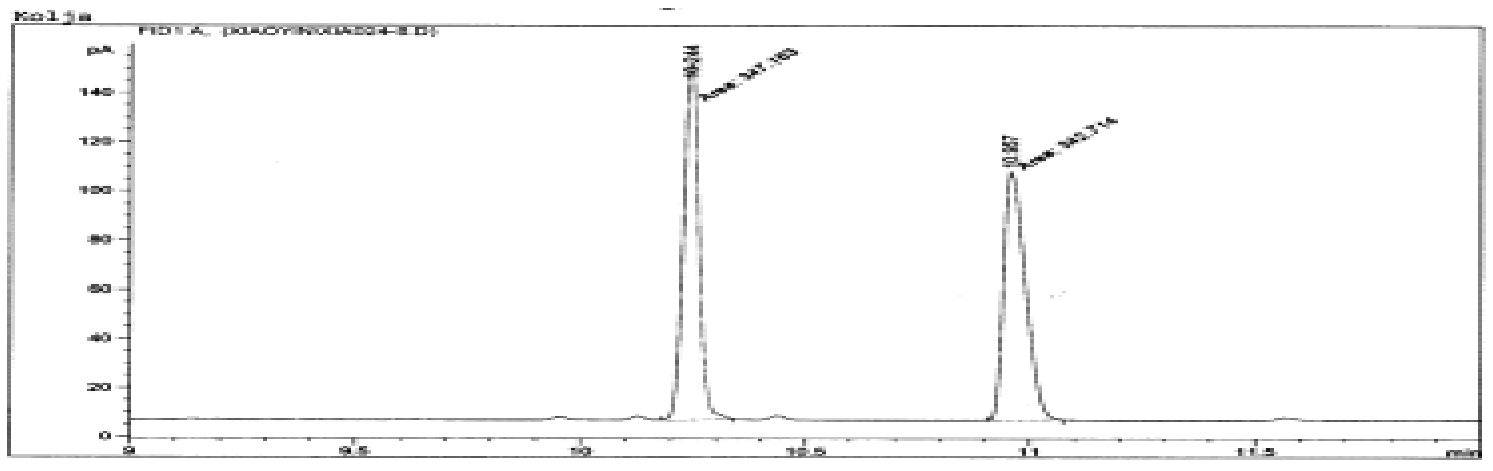


b)chiral

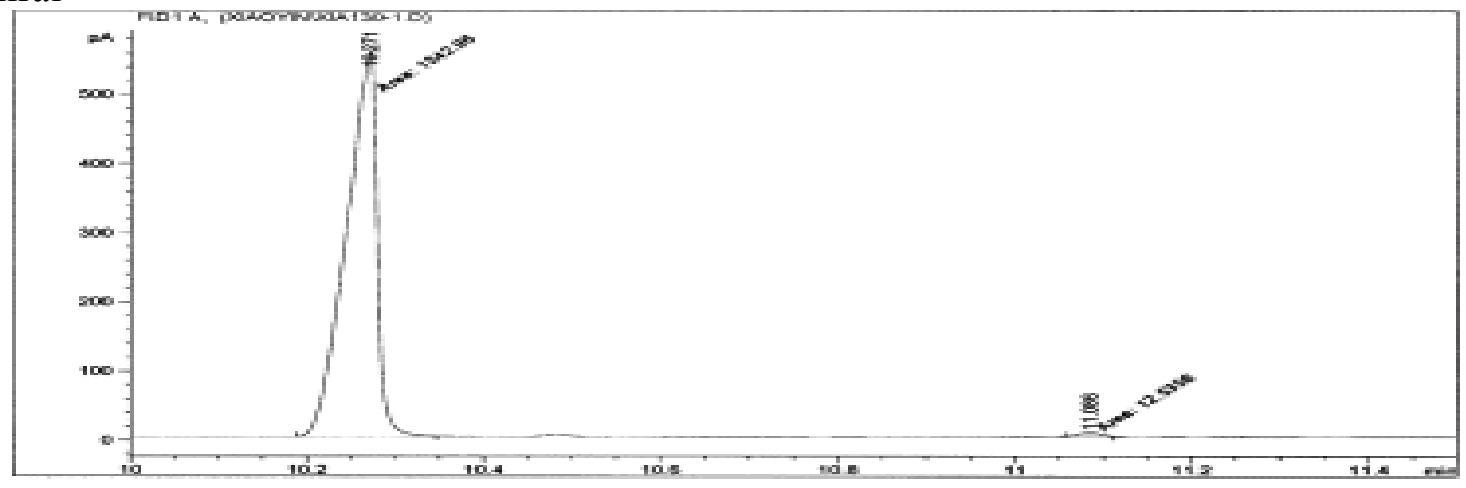

4-[(1R)-(2-Iodo-2-cyclopenten-1-yl)] phenyl methyl ether (5a)

$\mathrm{GC}\left(150{ }^{\circ} \mathrm{C}(100 \mathrm{~min})\right)$; TFA gamma-cyclodextrin, $\left.30.0 \mathrm{~m} \times 0.25 \mathrm{~mm}\right): t_{R} / \mathrm{min} 18.3(R), 18.9$ $(S) ; 96 \%$ ee.

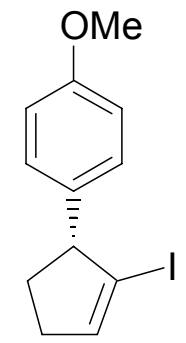

a) racemic

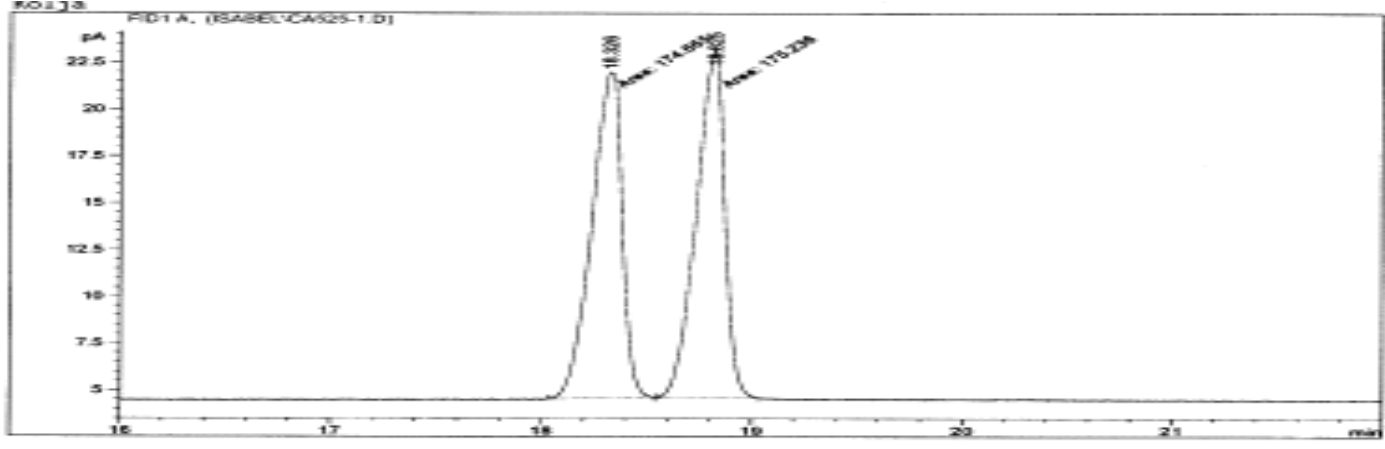

b) chiral

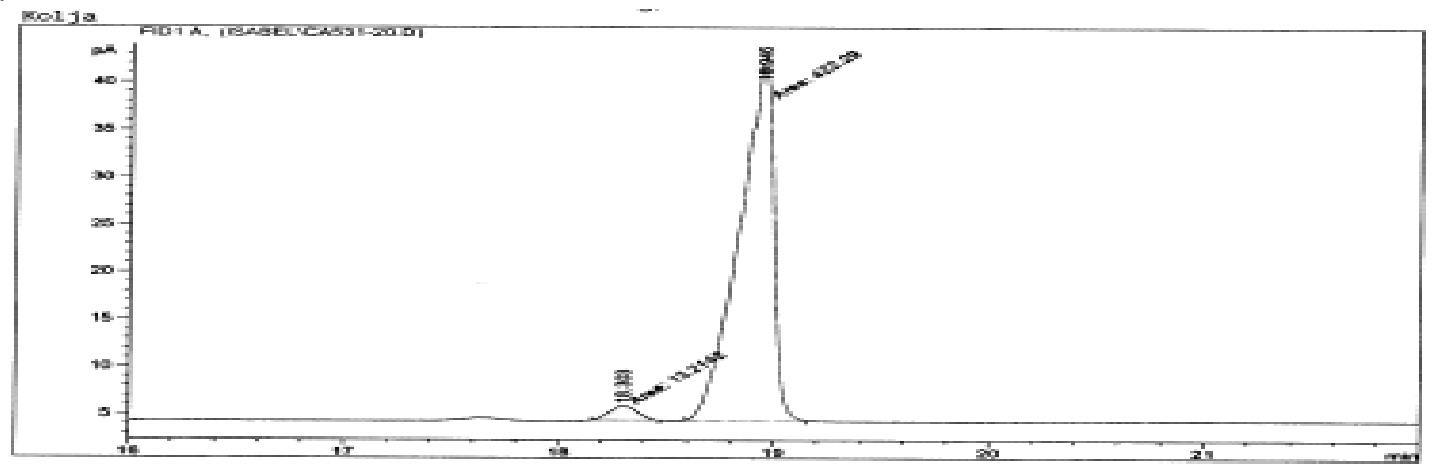

Ethyl 4-[(1R)-(2-iodo-2-cyclopenten-1-yl)] benzoate (5b):

GC (150 $\left.{ }^{\circ} \mathrm{C}(100 \mathrm{~min})\right)$; TFA gamma-cyclodextrin, $\left.30.0 \mathrm{~m} \mathrm{x} 0.25 \mathrm{~mm}\right): t_{R} / \mathrm{min} 81.5(R), 85.2$ (S); $92 \%$ ee. 
a) racemic
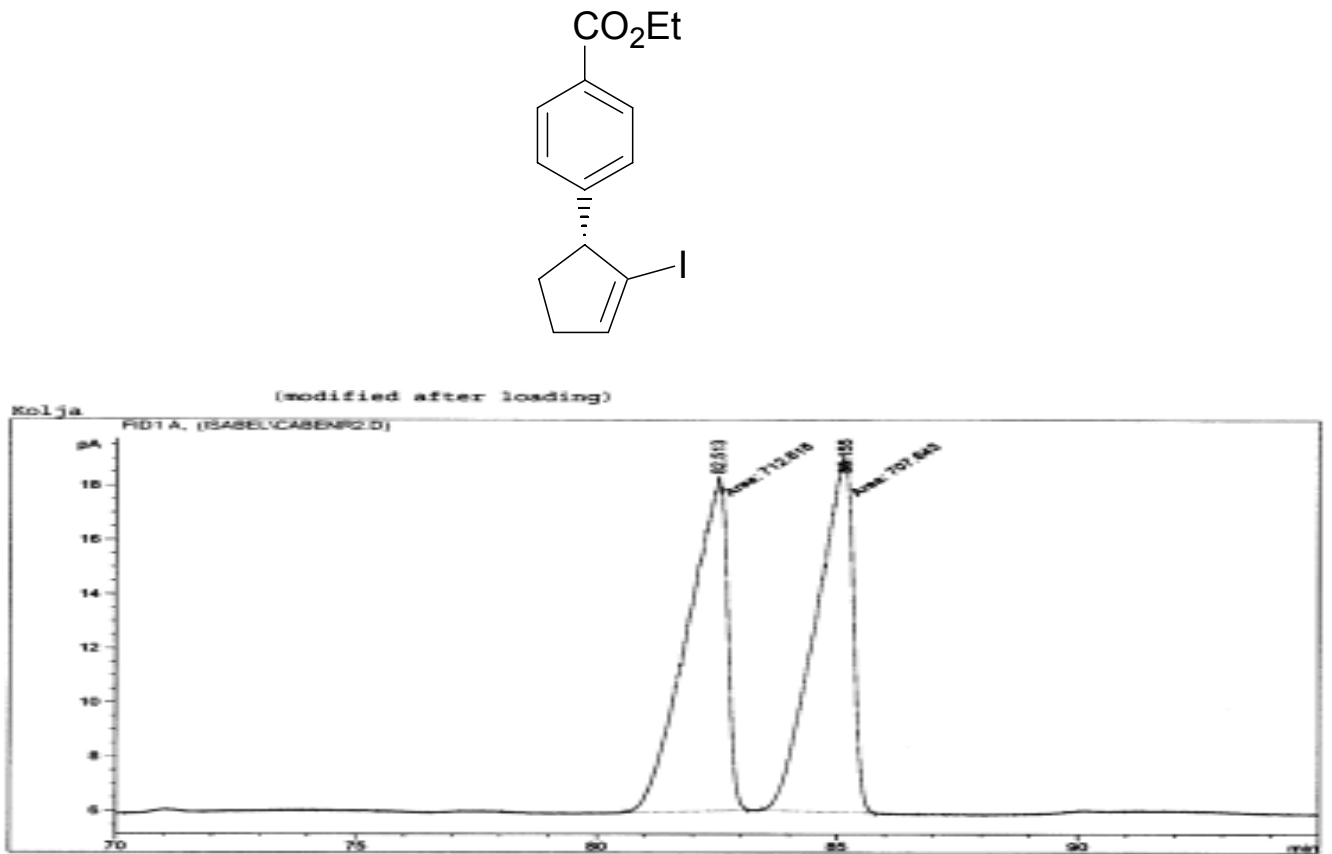

b) chiral

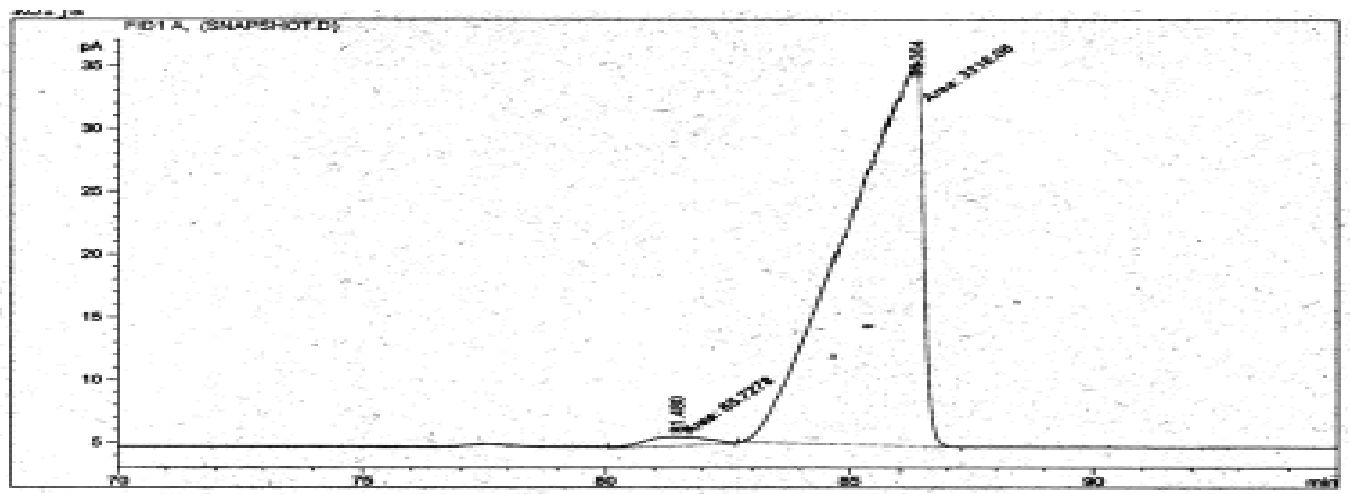

Ethyl 4-[(1R)-2-iodocyclohex-2-en-1-yl]beozoate (5c):

HPLC (Chiralcel AD, $0.46 \mathrm{~cm} \times 25 \mathrm{~cm}$; heptane : $i \mathrm{PrOH}=99: 1,0.5 \mathrm{ml} / \mathrm{min}$ ): $t_{R} / \mathrm{min}=18.3$ (S), $19.8(R) ; 98 \%$ ee.

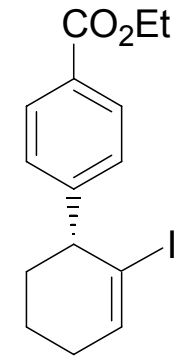

a): racemic

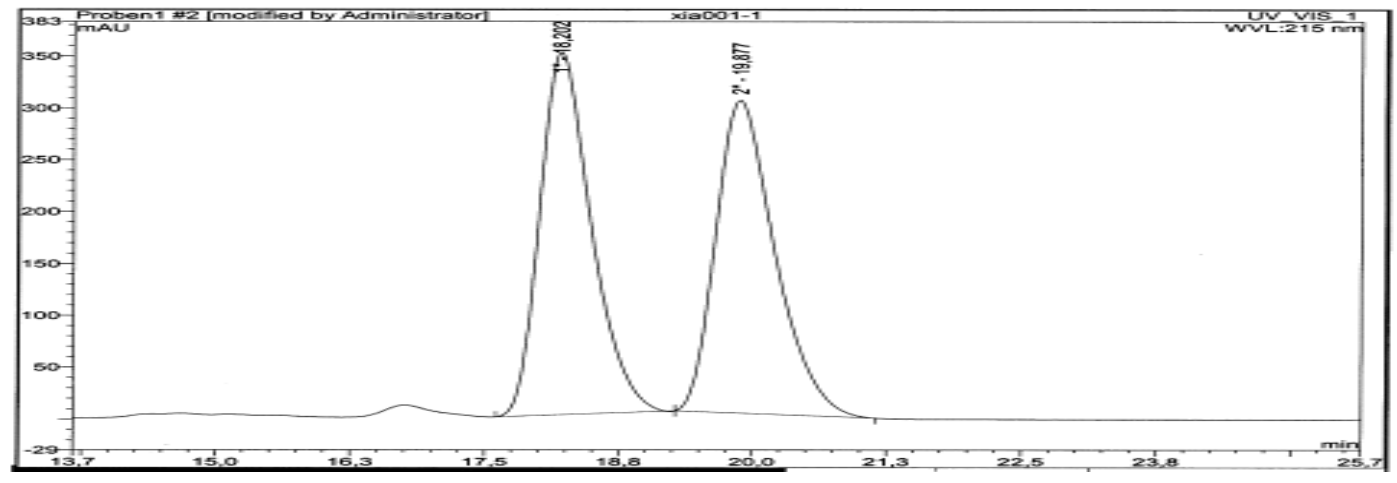


b) chiral

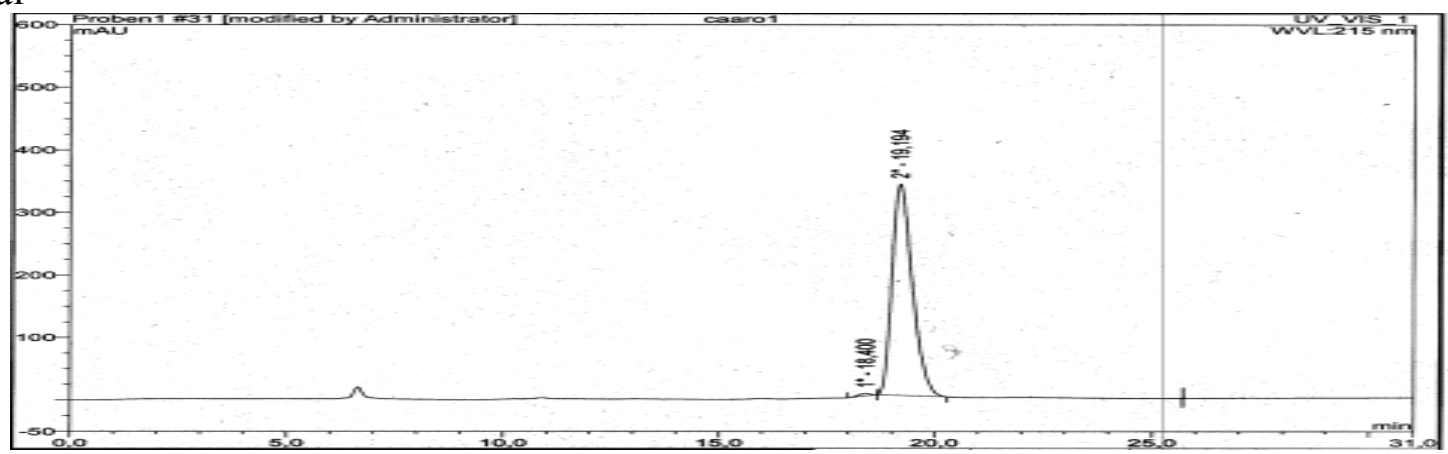

4-[(1R)-2-Iodocyclohex-2-en-1-yl]benzonitrile (5d):

GC $\left(130{ }^{\circ} \mathrm{C}(100 \mathrm{~min})\right)$; TFA gamma-cyclodextrin, $\left.30.0 \mathrm{~m} \mathrm{x} 0.25 \mathrm{~mm}\right): t_{R} / \min 33.7(R), 34.9$ $(R) ; 96 \%$ ee.<smiles>N#Cc1ccc(C2CCCC=C2I)cc1</smiles>

a) racemic

b) chiral
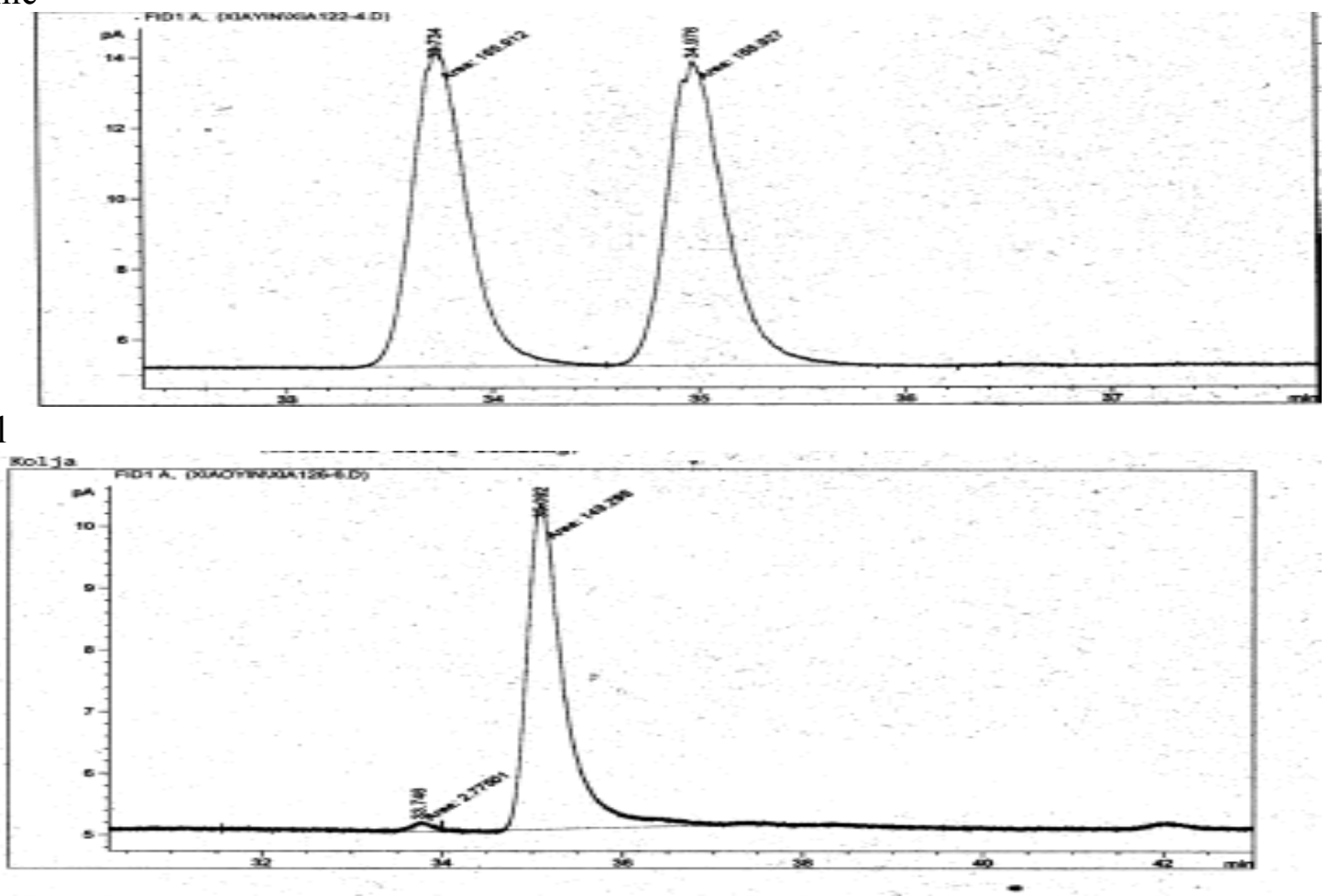

1-[(1R)-2-Iodocyclohex-2-en-1-yl]-4-(trifluoromethyl)benzene (5e):

$\mathrm{GC}\left(130{ }^{\circ} \mathrm{C}(100 \mathrm{~min})\right)$; TFA gamma-cyclodextrin, $30.0 \mathrm{~m}$ x $\left.0.25 \mathrm{~mm}\right): t_{R} / \mathrm{min} 24.6(S), 26.0$ $(R) ; 94 \%$ ee. 


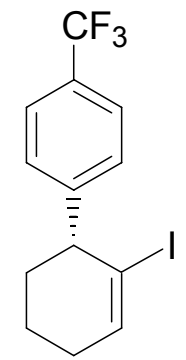

a) racemic



b) chiral

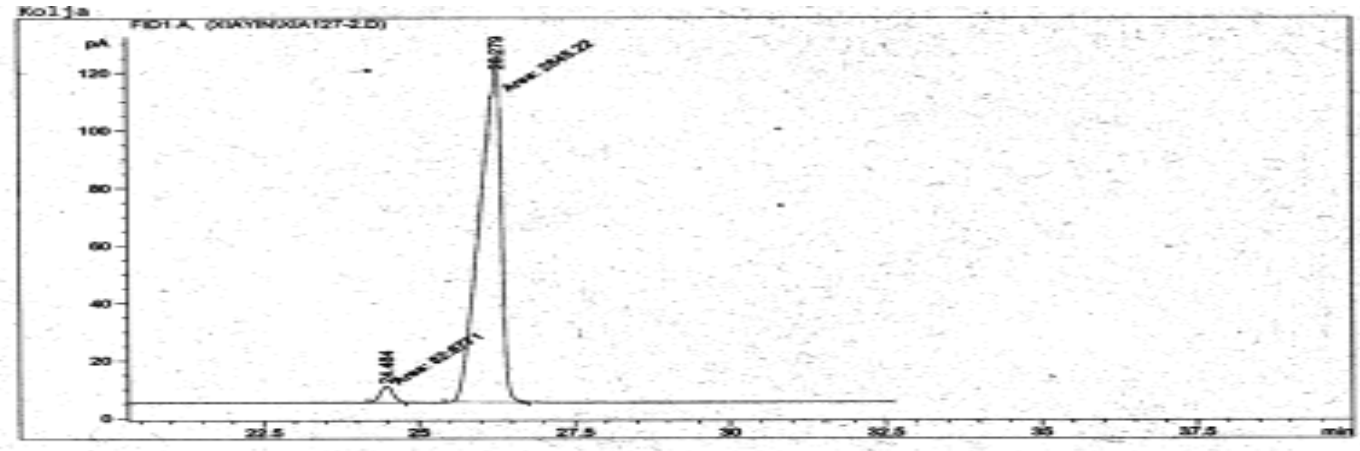

1-Iodo-4-[(1R)-2-iodocyclohex-2-en-1-yl]benzene (5f):

$\mathrm{GC}\left(150{ }^{\circ} \mathrm{C}(100 \mathrm{~min})\right)$; TFA gamma-cyclodextrin, $\left.30.0 \mathrm{~m} \times 0.25 \mathrm{~mm}\right): t R / \min 78.7(R), 82.2$ (S); $97 \%$ ee.

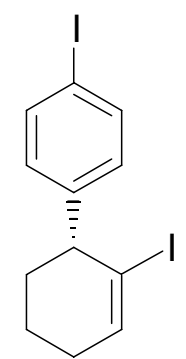

a) racemic

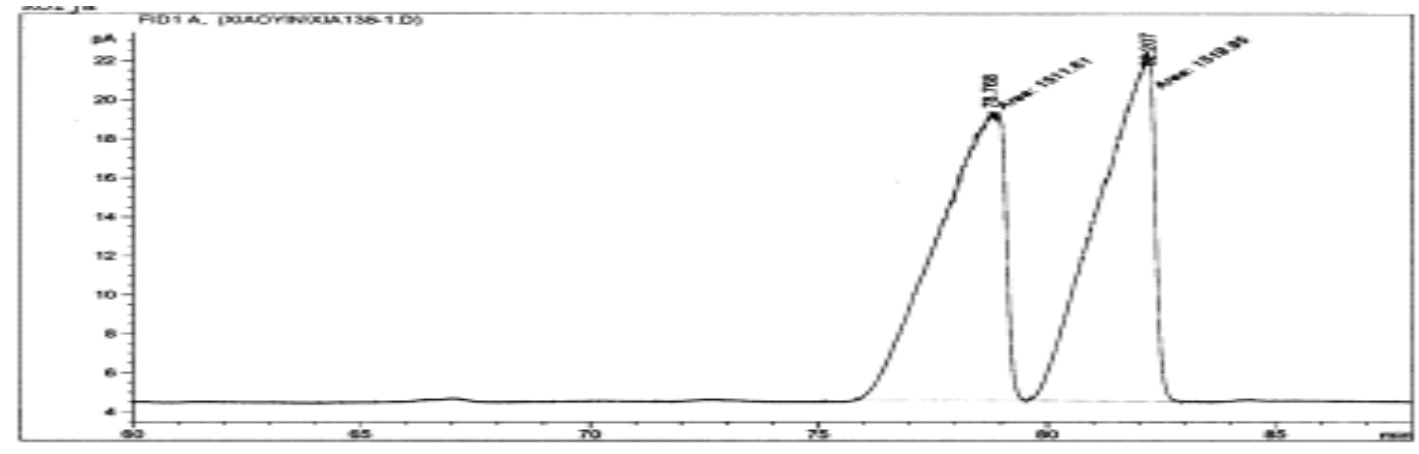


b) chiral

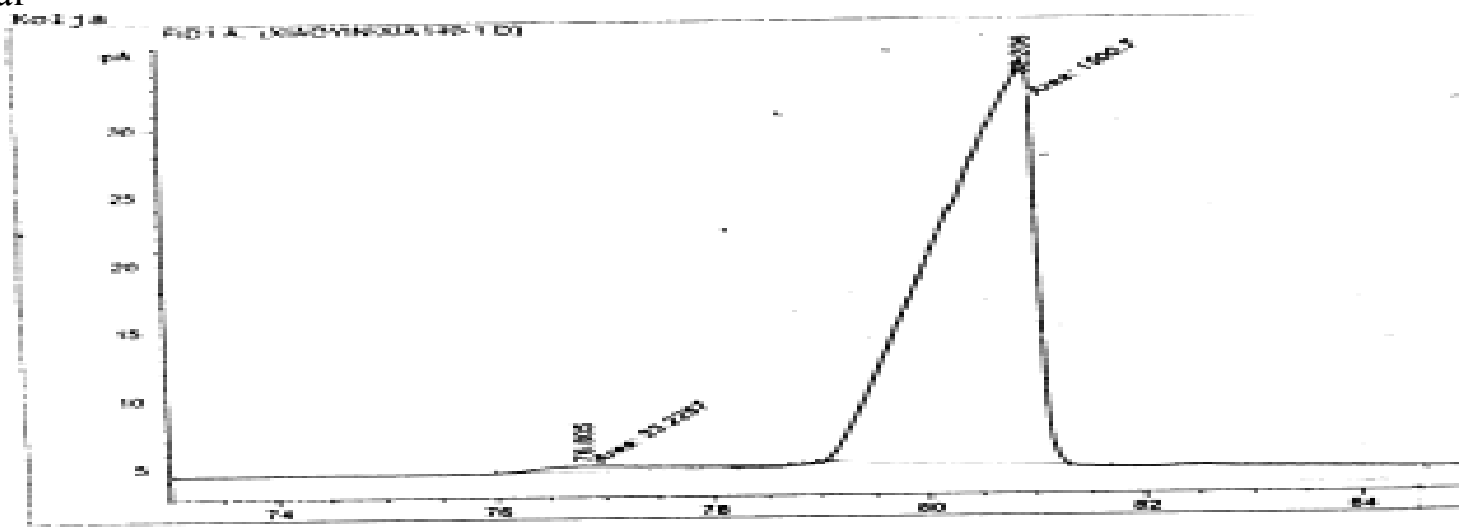

\section{1-Bromo-4-[(1R)-2-iodocyclohex-2-en-1-yl]benzene (5g):}

$\mathrm{GC}\left(130{ }^{\circ} \mathrm{C}(100 \mathrm{~min})\right)$; TFA gamma-cyclodextrin, $\left.30.0 \mathrm{~m} \times 0.25 \mathrm{~mm}\right): t_{R} / \mathrm{min} 123.7(S)$, $133.6(R) ; 96 \%$ ee.

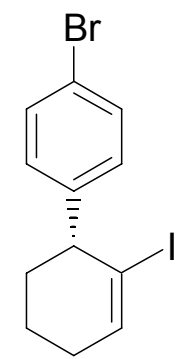

a) racemic

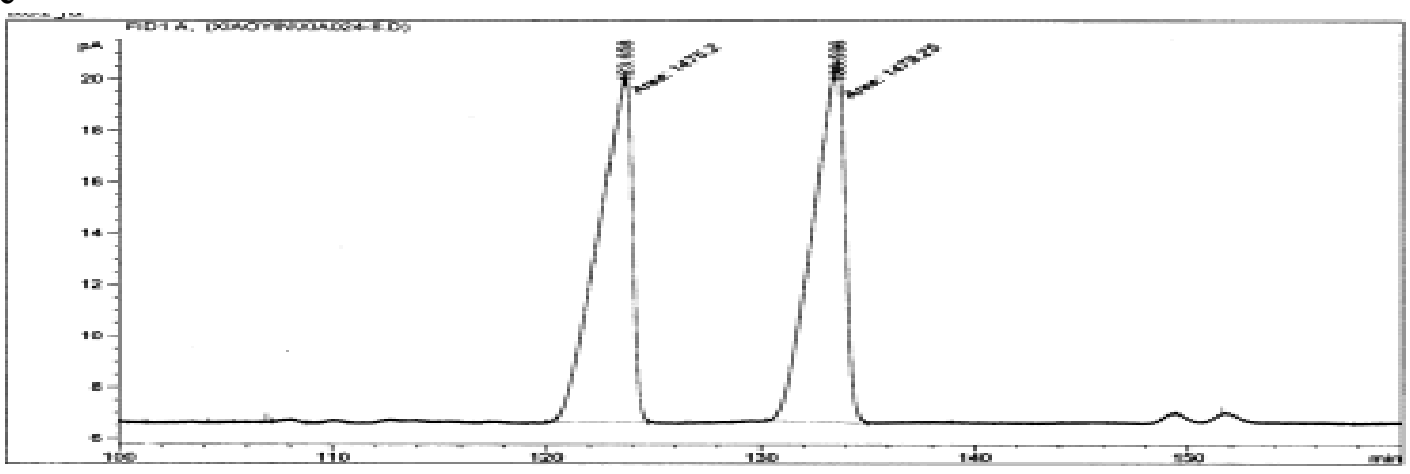

b) chiral

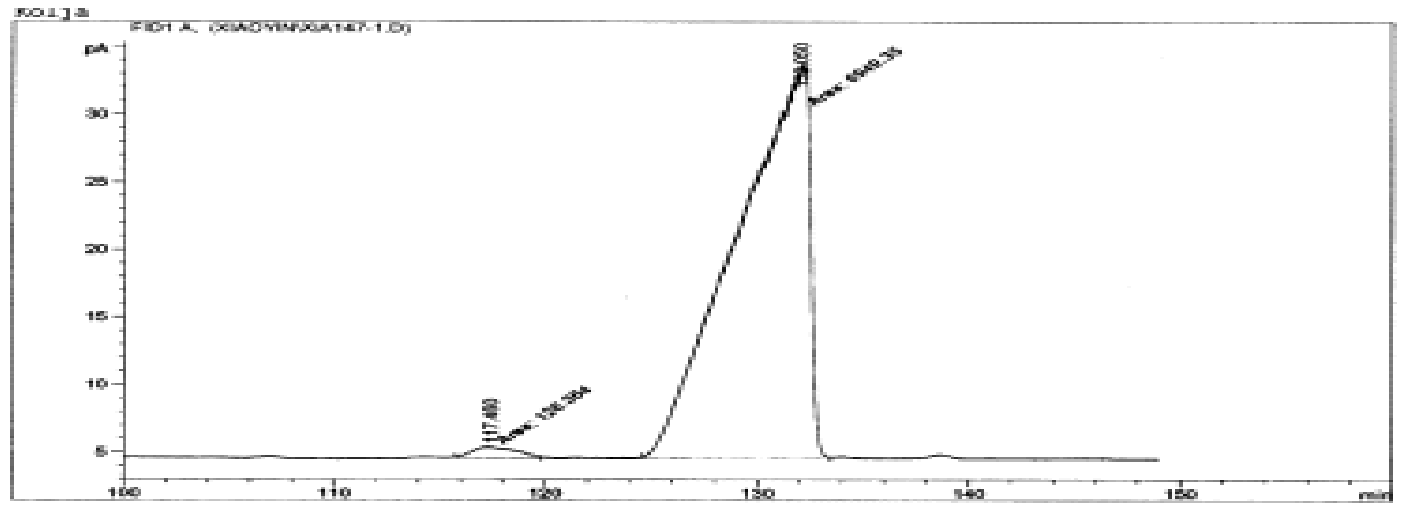

1-[(1R)-2-Iodocyclohex-2-en-1-yl]-4-methoxybenzene (5h):

$\mathrm{GC}\left(130{ }^{\circ} \mathrm{C}(100 \mathrm{~min})\right)$; TFA gamma-cyclodextrin, $30.0 \mathrm{~m}$ x $\left.0.25 \mathrm{~mm}\right): t_{R} / \mathrm{min} 83.3(S), 87.8$ $(R) ; 95 \%$ ee. 


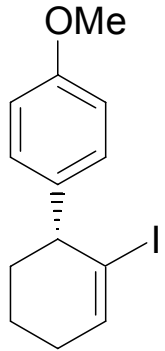

a) racemic

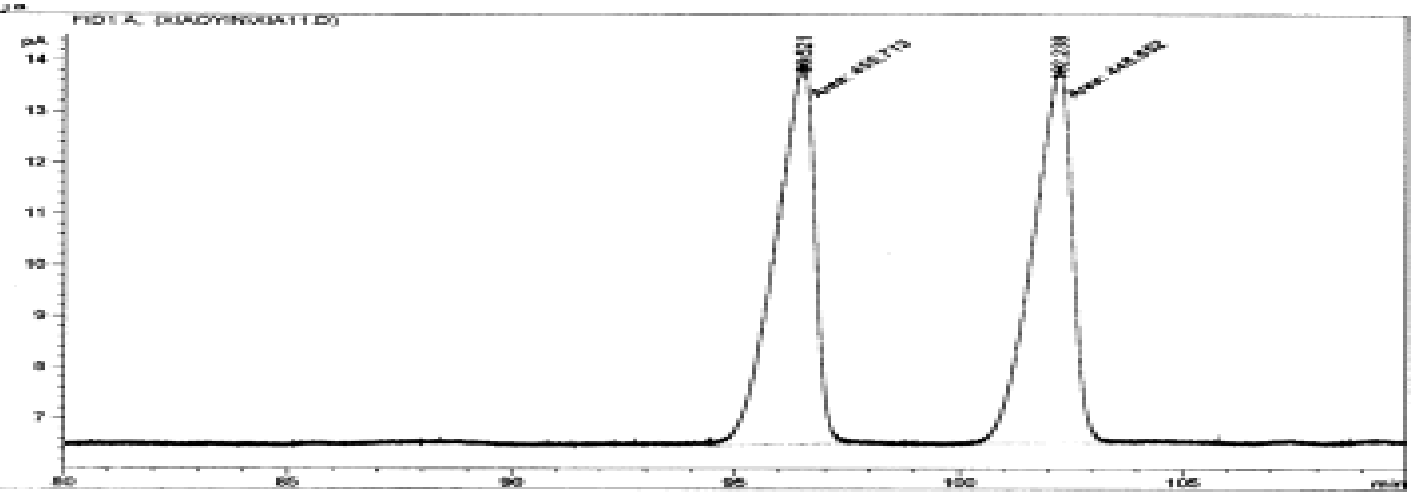

b) chiral

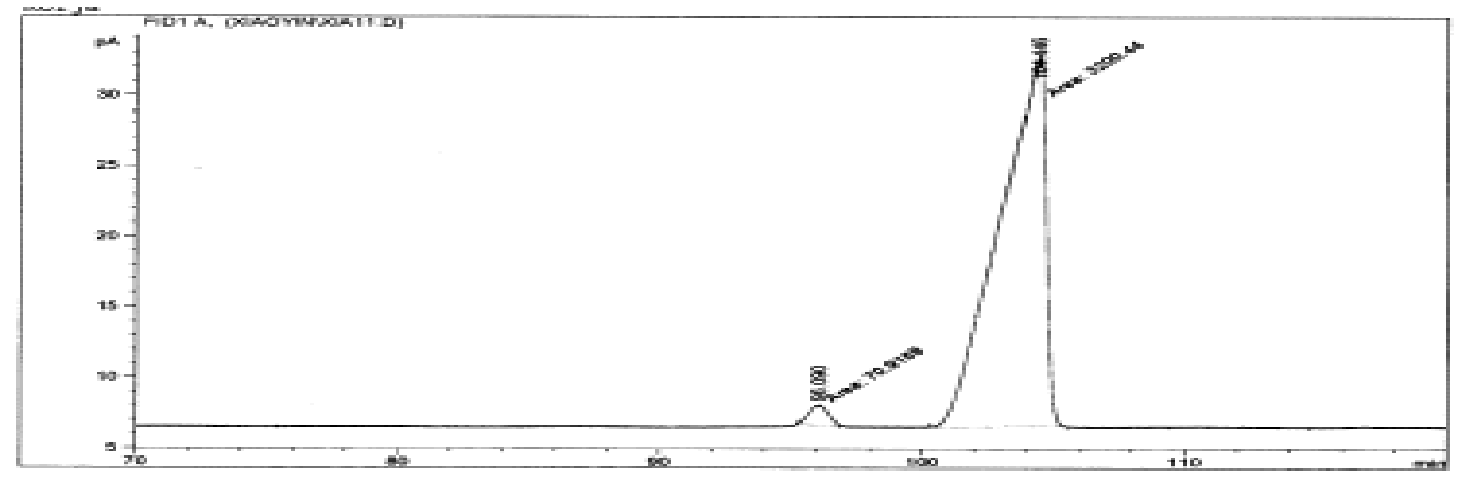

1-\{4-[(1R)-2-Iodocyclohex-2-en-1-yl]phenyl\}ethanone (5i):

HPLC (Chiralcel AD, $0.46 \mathrm{~cm} \times 25 \mathrm{~cm}$; heptane $: i \operatorname{PrOH}=99: 1,0.4 \mathrm{ml} / \mathrm{min}$ ): $t_{R} / \mathrm{min}=37.4$ $(S), 43.3(R) ; 89 \%$ ee.<smiles>CC(=O)c1ccc([C]2CCCC=C2I)cc1</smiles>

a) racemic 
b) chiral
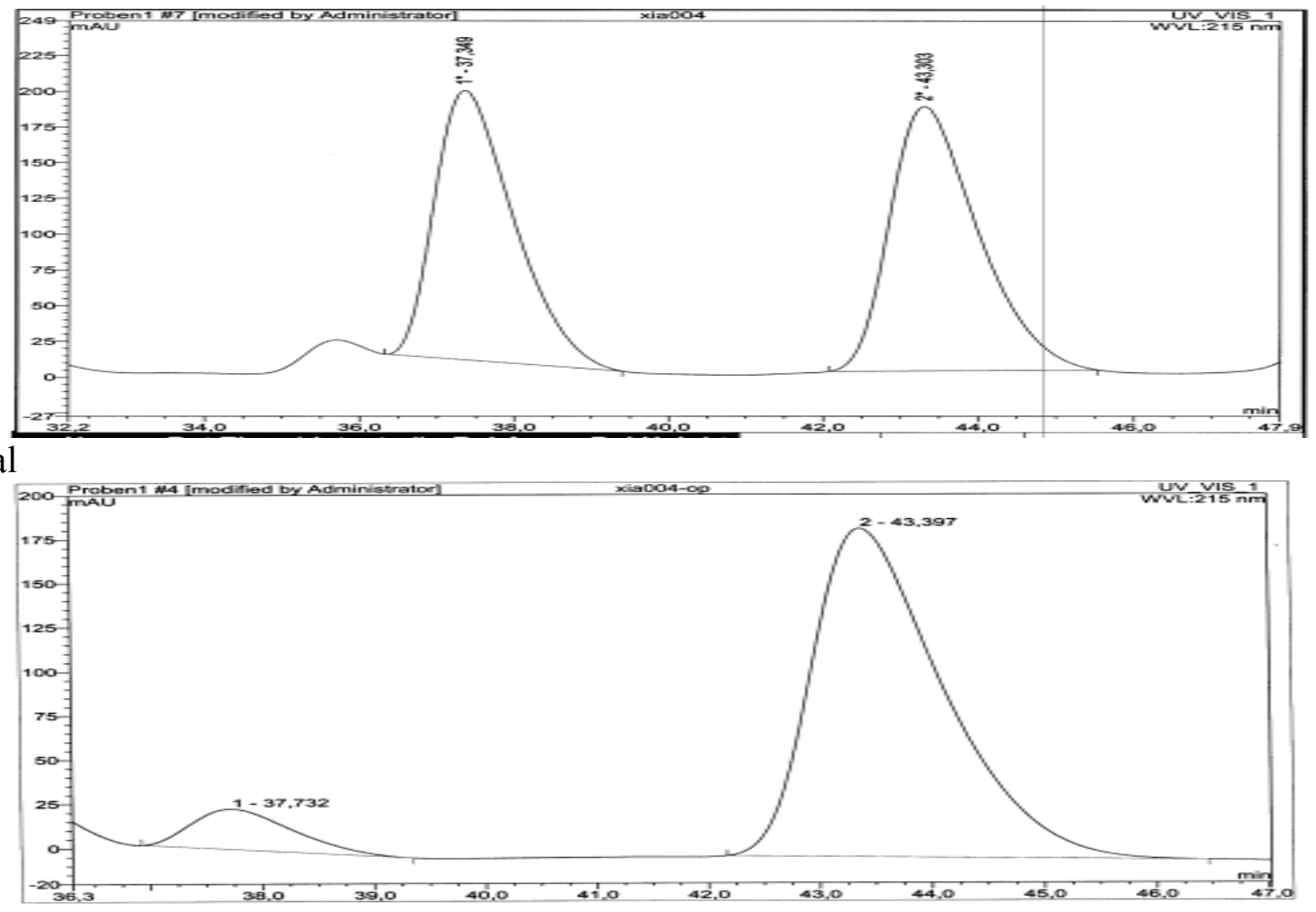

1-[(1R)-2-Iiodocyclohex-2-en-1-yl]-3-methoxybenzene $(\mathbf{5 j})$ :

HPLC (Chiralcel OD-H, $0.46 \mathrm{~cm} \times 25 \mathrm{~cm}$; heptane $: i \mathrm{PrOH}=99: 1,0.2 \mathrm{ml} / \mathrm{min}$ ): $t_{R} / \mathrm{min}=31.5$ $(S), 38.1(R) ; 92 \%$ ee.

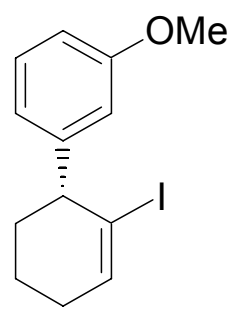

a) racemic

b) chiral

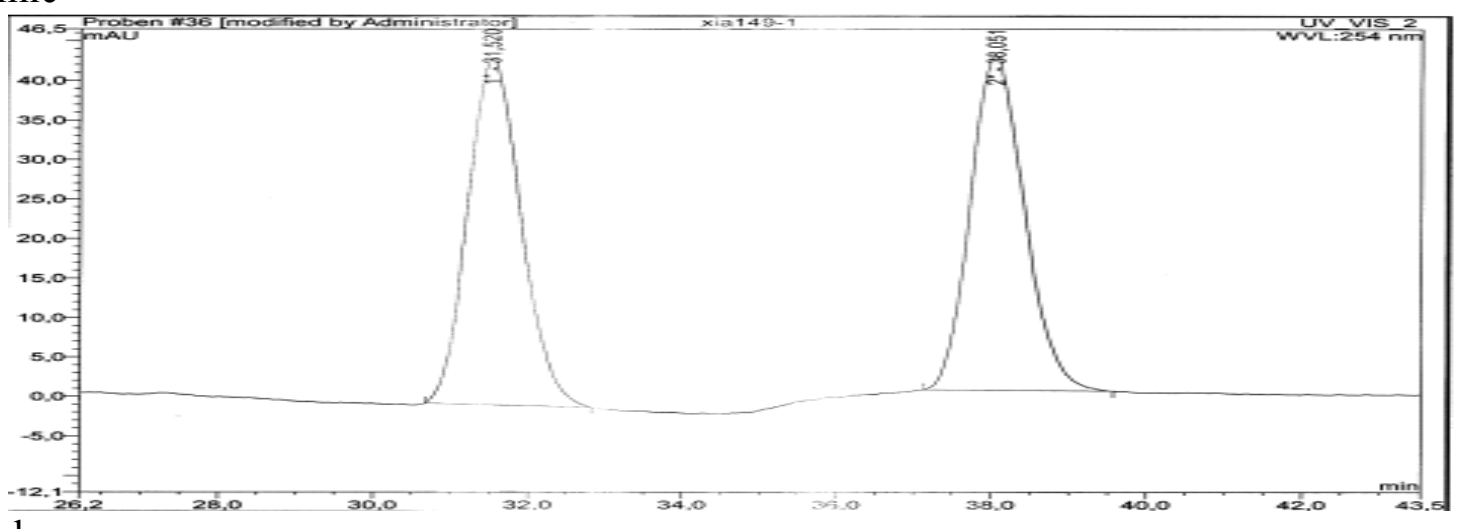




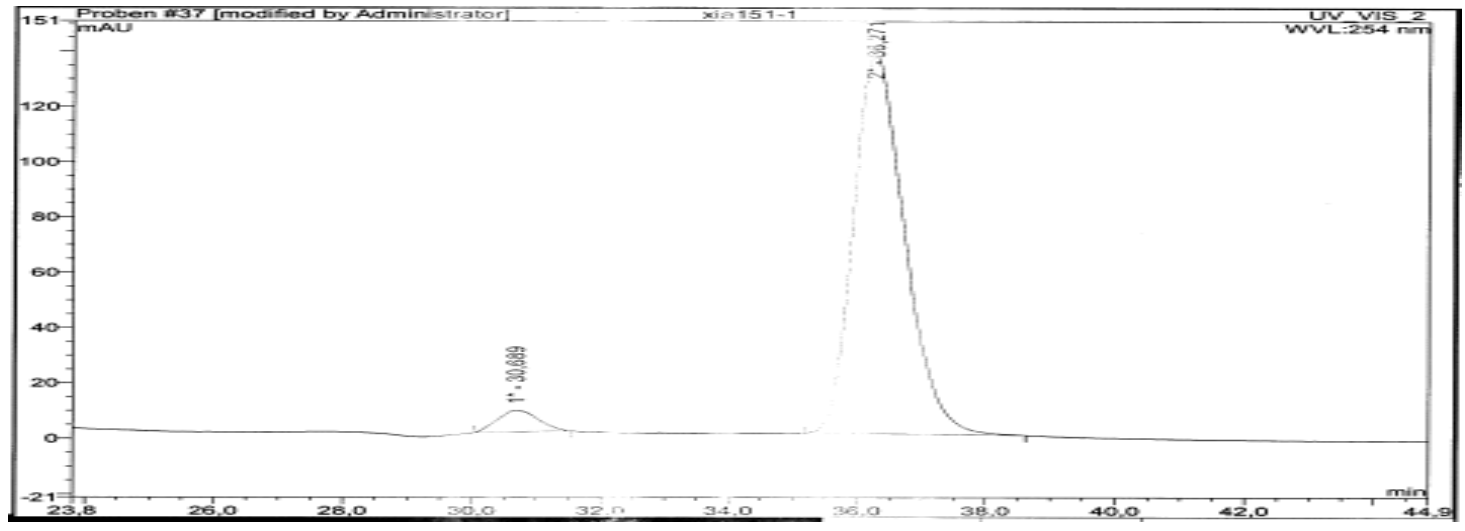

Ethyl 4-[(1R,2E)-1-methylhept-2-en-1-yl]benzoate (8):

HPLC (OD, $0.46 \mathrm{~cm} \times 25 \mathrm{~cm}$; heptane : $i \mathrm{PrOH}=99: 1,0.2 \mathrm{ml} / \mathrm{min}): t_{R} / \mathrm{min}=21.8(R), 23.8$ $(S) ; 95 \%$ ee.

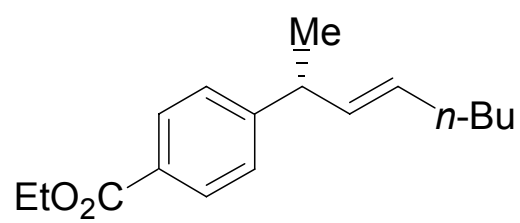

a) racemic

b) chiral
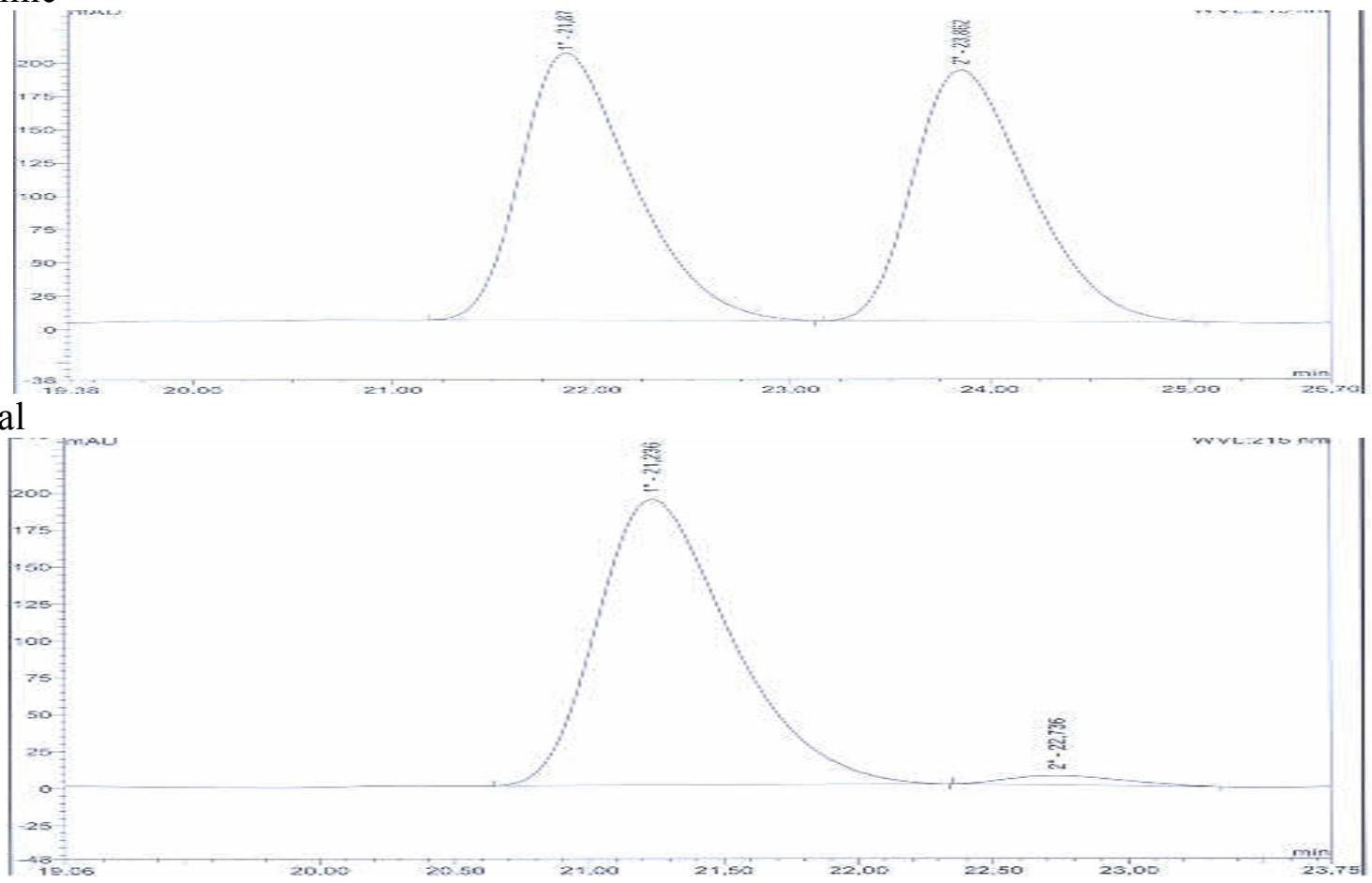

Reference:

[1] Demay, S.; Harms, K.; Knochel, P. Tetrahedron Lett. 1999, 40, 4981.

[2] Harrington-Frost, N.; Leuser, H.; Calaza, M. I.; Knochel, P. Org. Lett. 2003, 5, 2111.

[3] Lin, H.-S.; Paquette, L. A. Synth. Commun. 1994, 24, 2503.

[4] (a) Cano, A.; Cuenca, T.; Galakov, M.; Rodríguez, G. M.; Royo, P.; Cardin, C. J.; Convery, M. A. J. Organomet. Chem. 1995, 493, 17. (b) Negishi, E.; Swanson, D. R.; Rousset, C. J. J. Org. Chem. 1990, 55, 5406.

[5] Zhdanov, R. I.; Zhenodarova, S. M. synthesis, 1975, 222. 


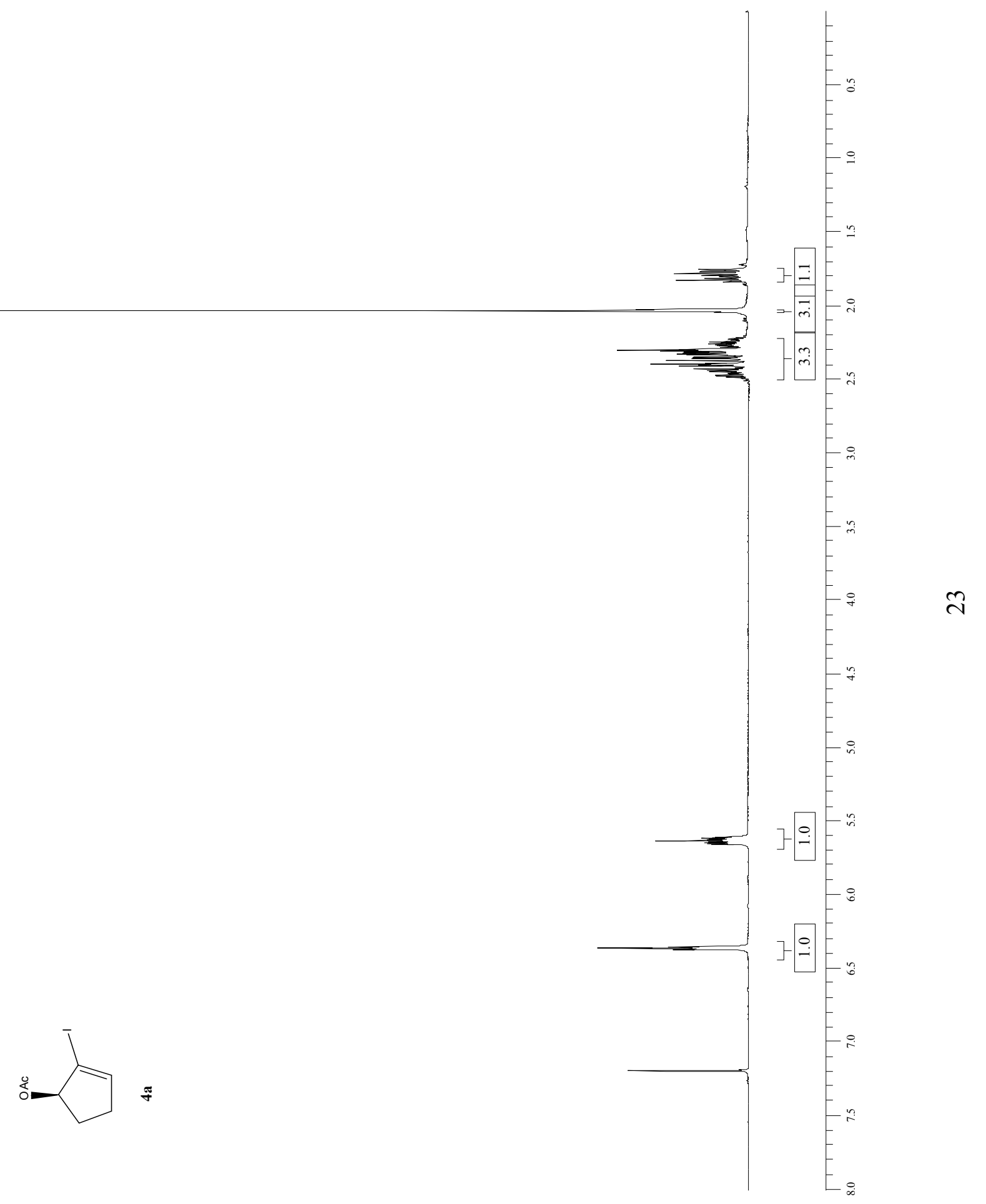




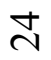

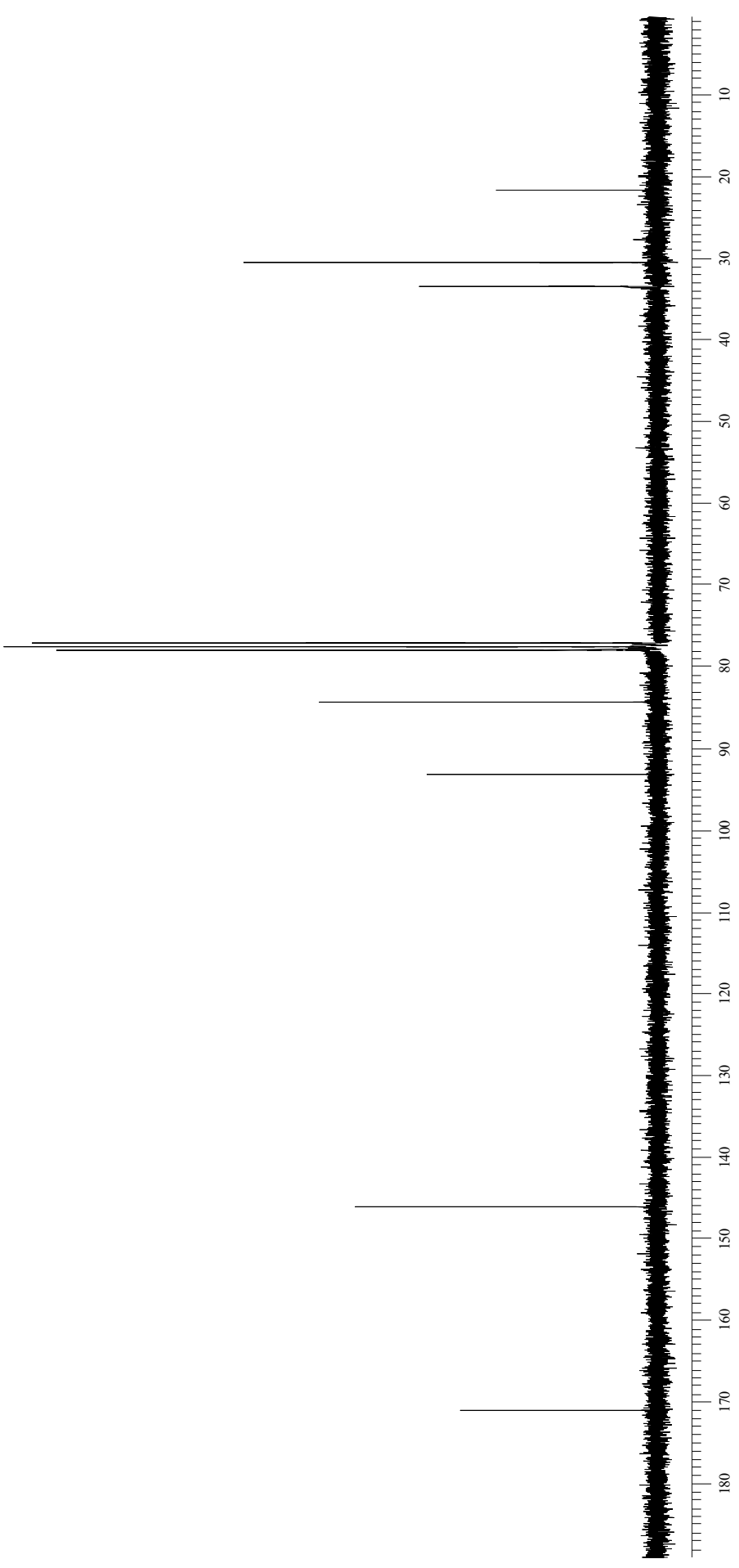

$\stackrel{+}{\sim}$

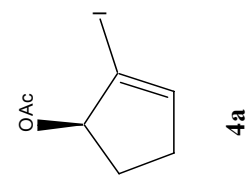




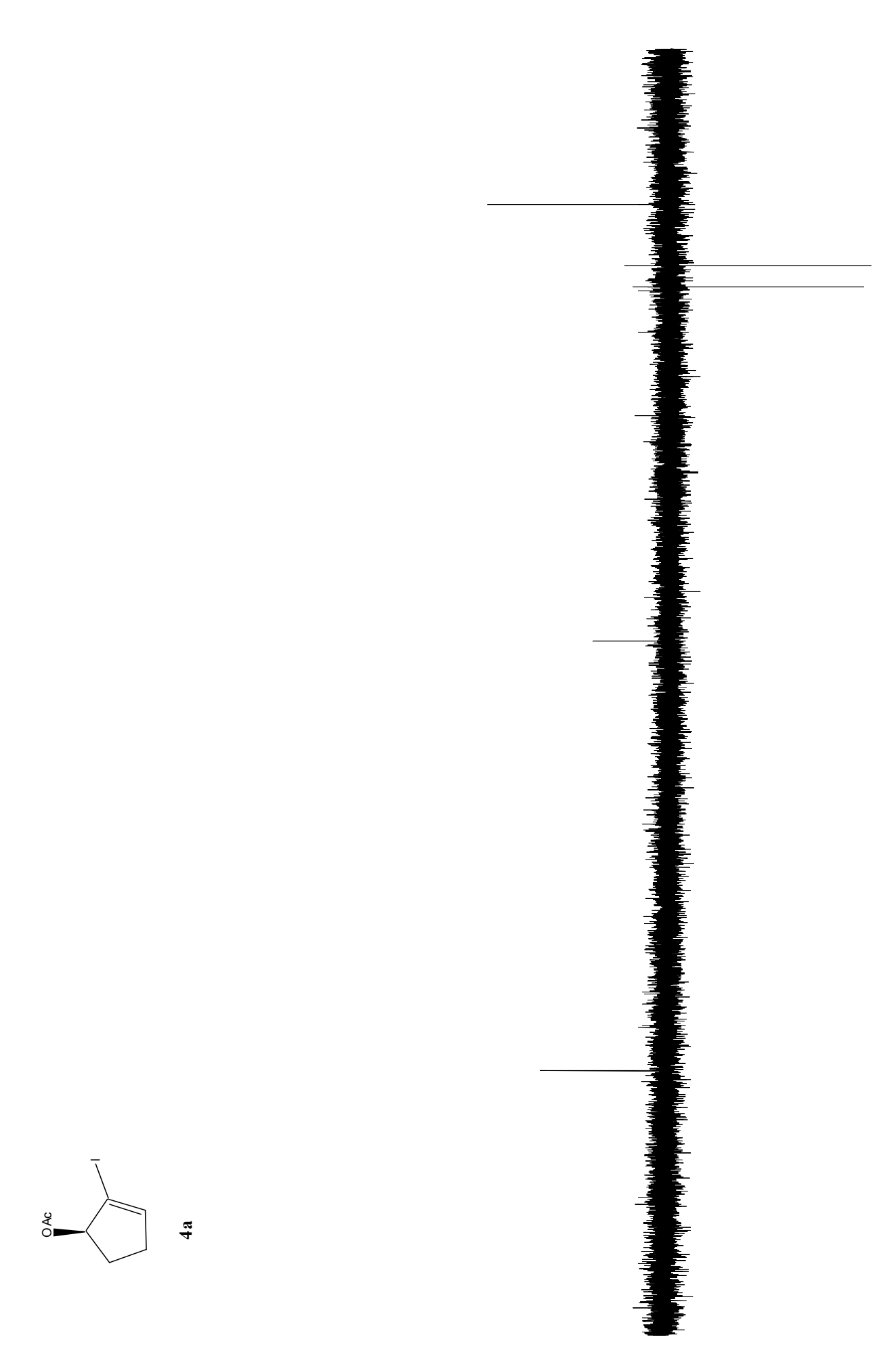




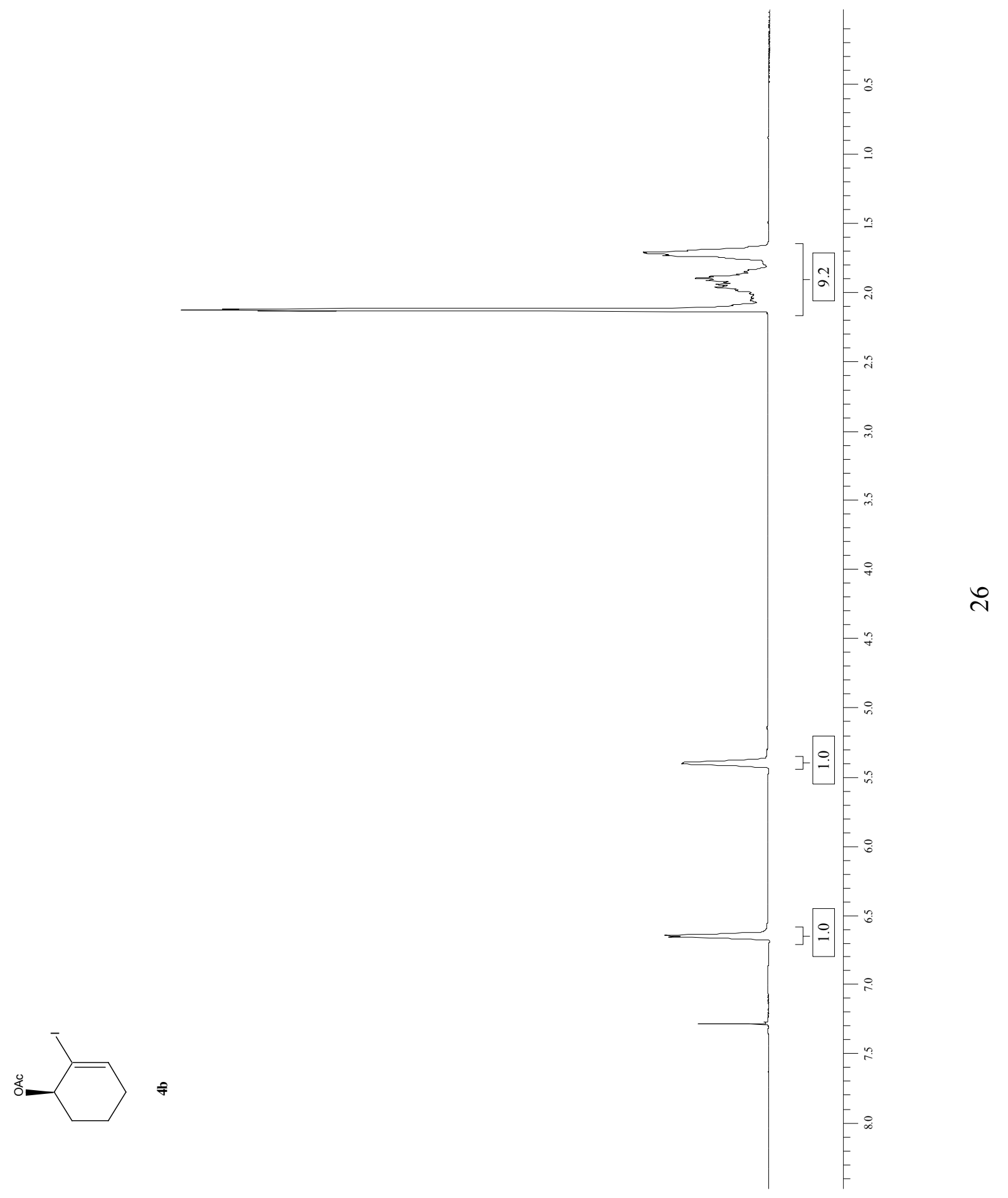




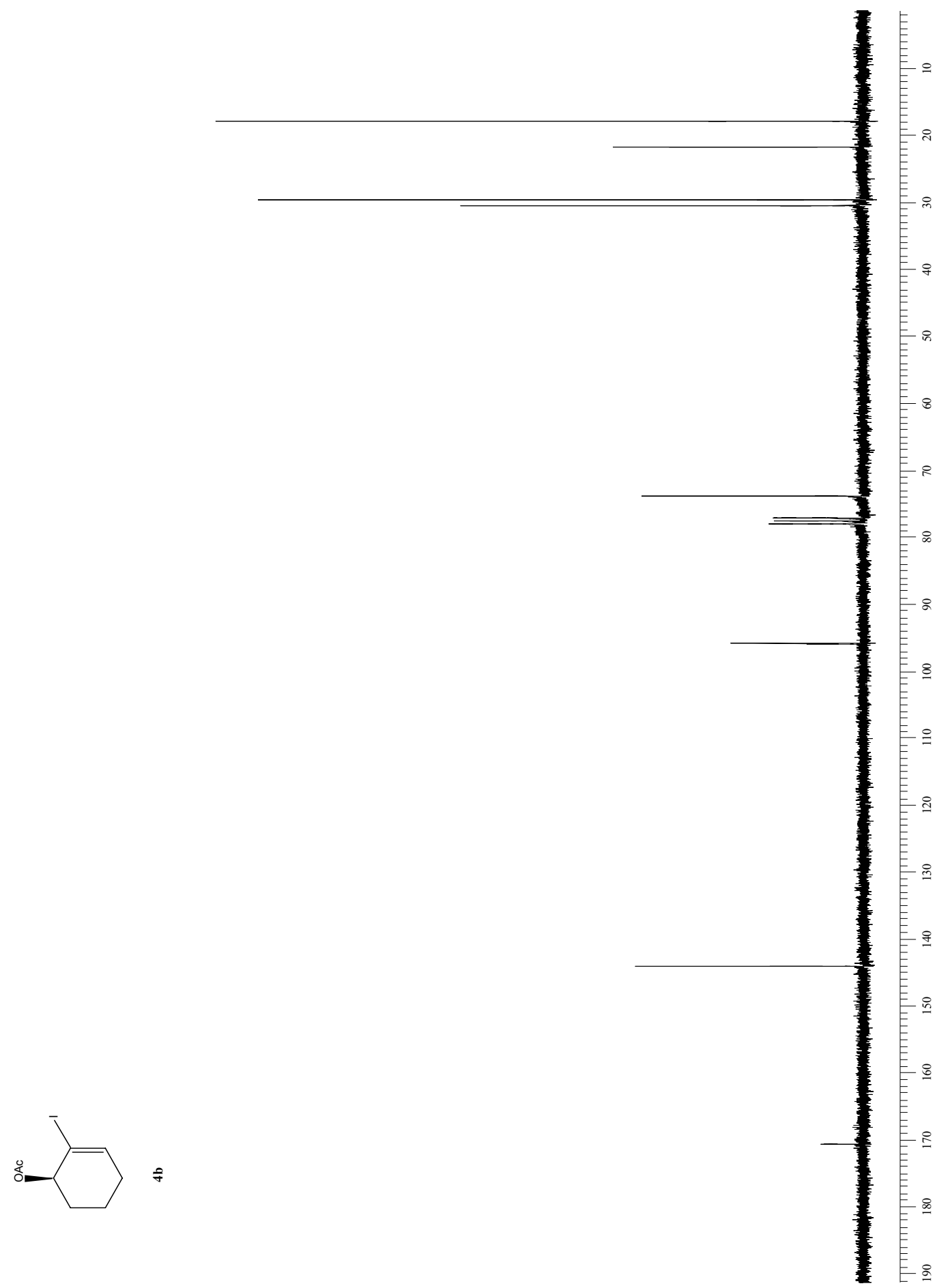


$\stackrel{\infty}{\sim}$

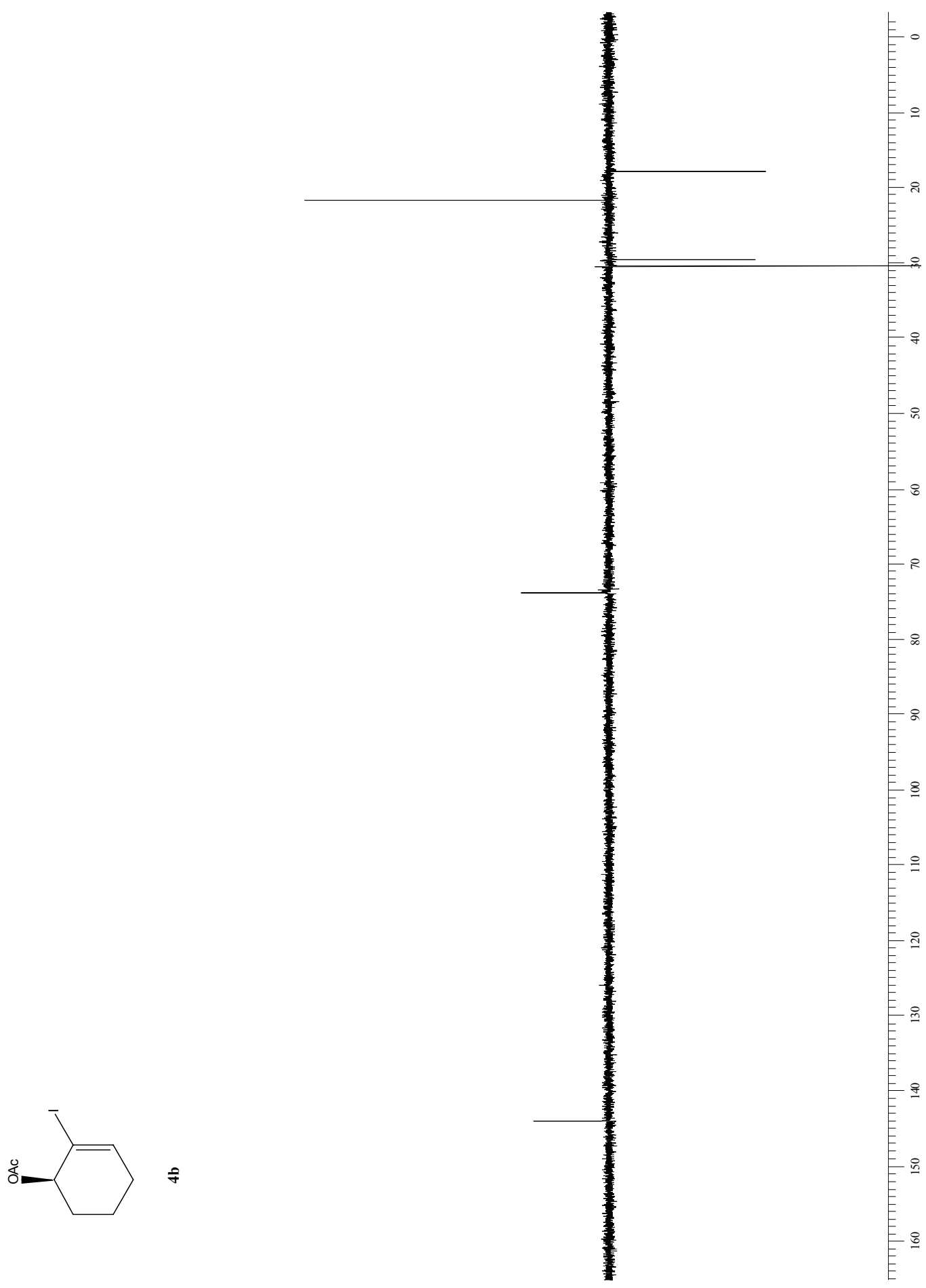


ิ

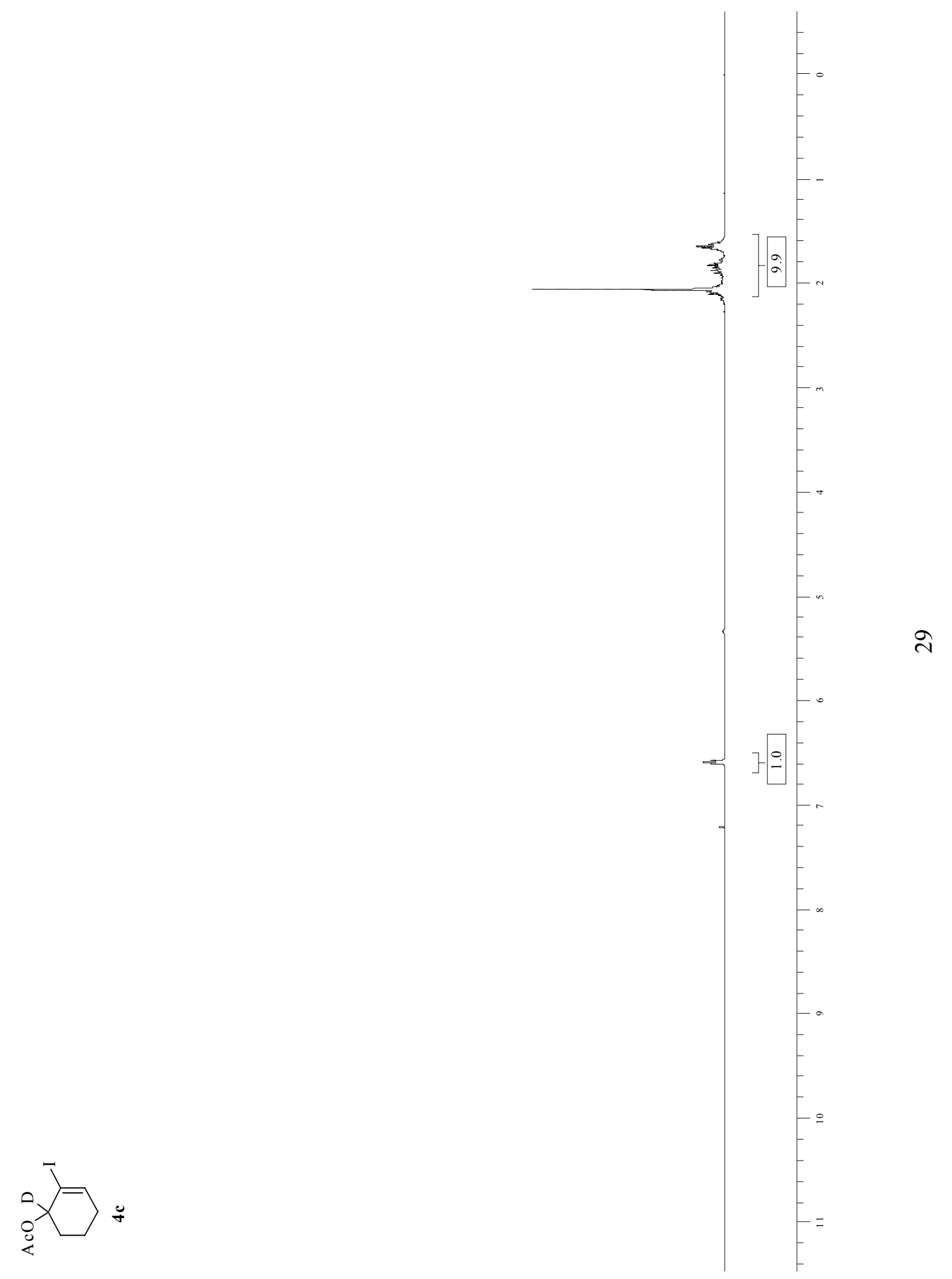


요

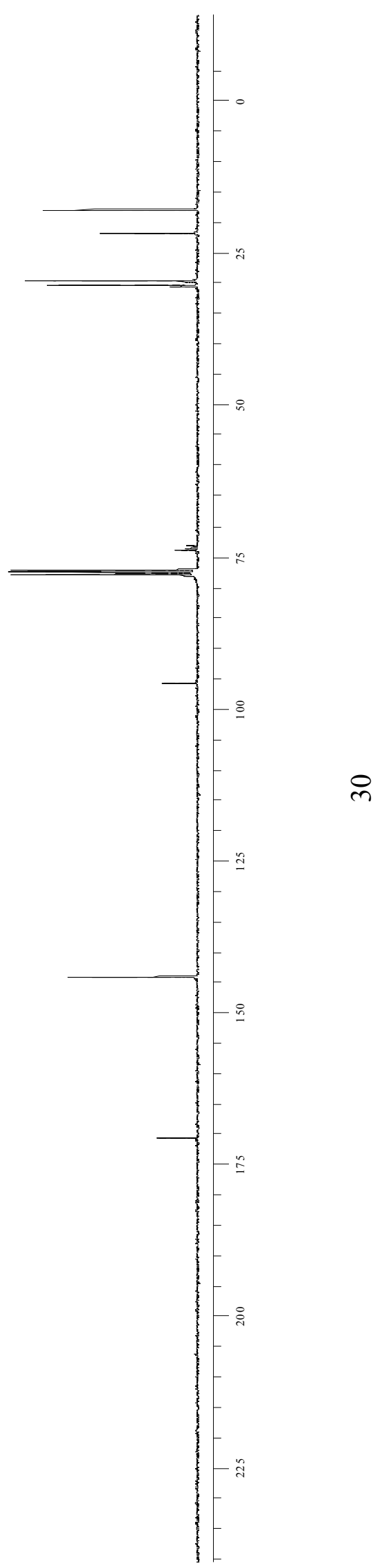


$\bar{m}$

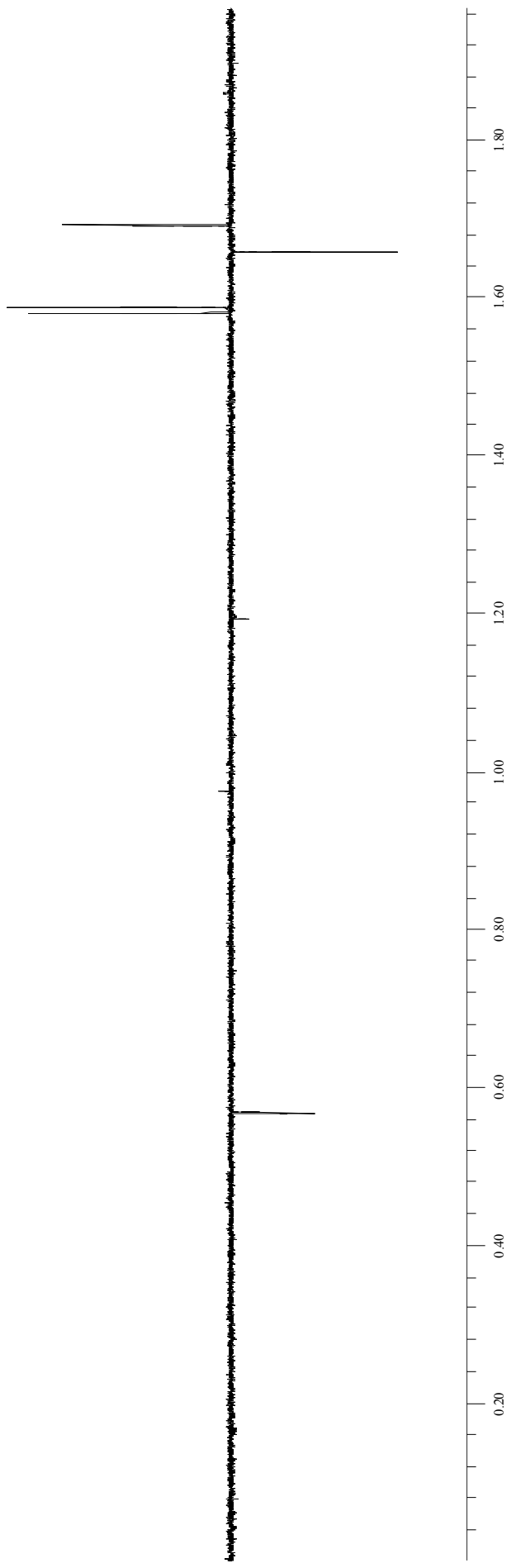




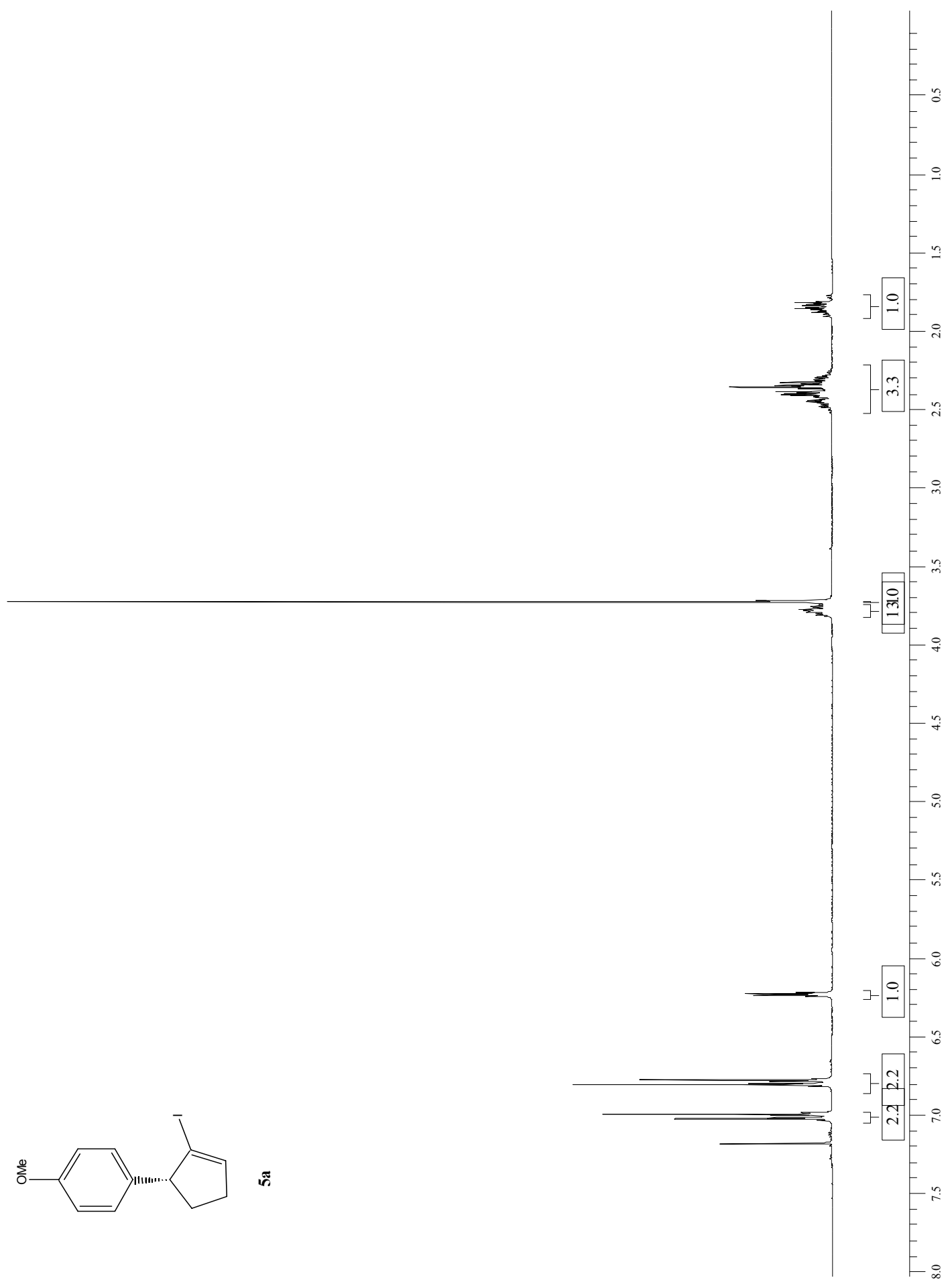




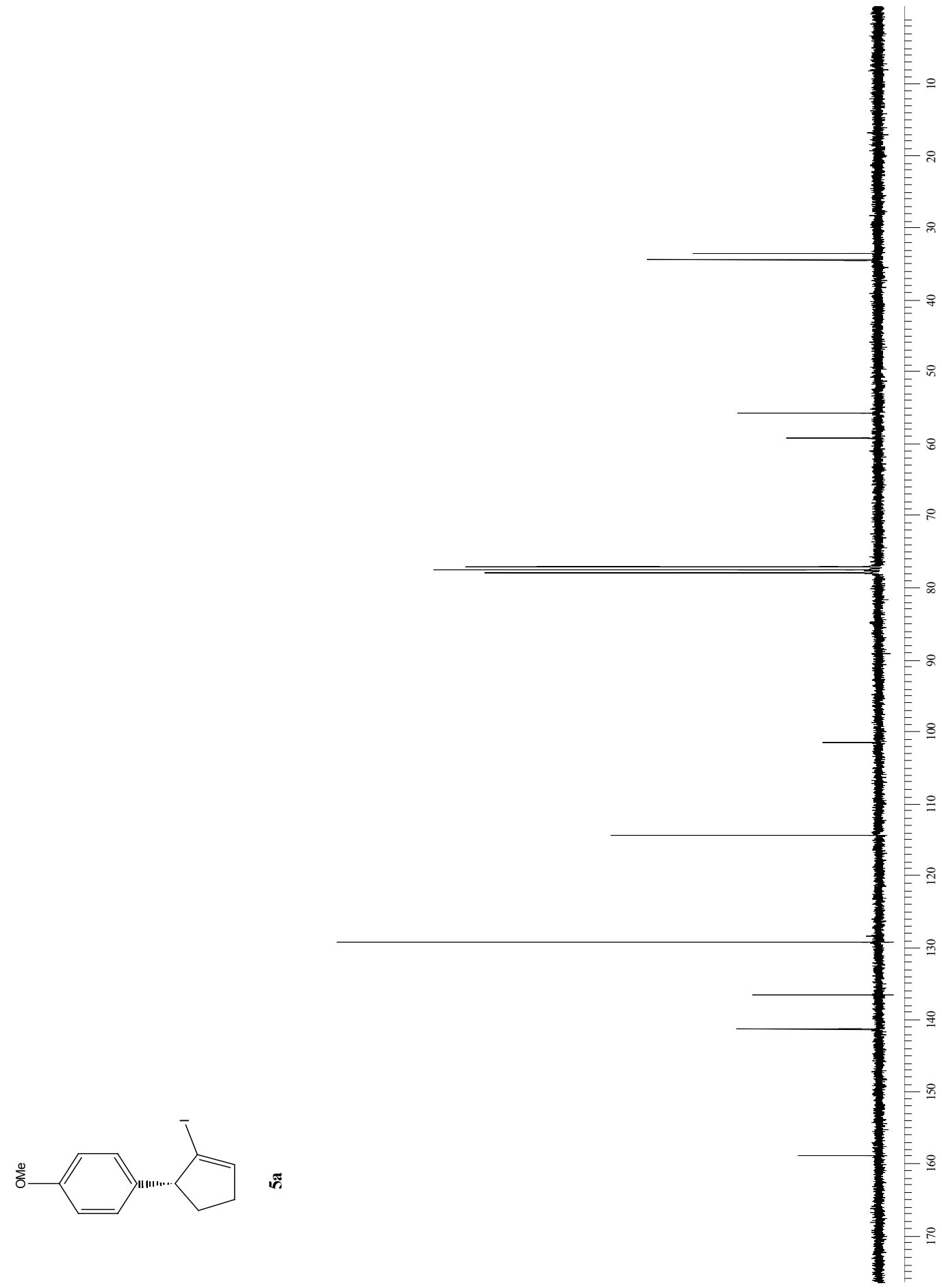




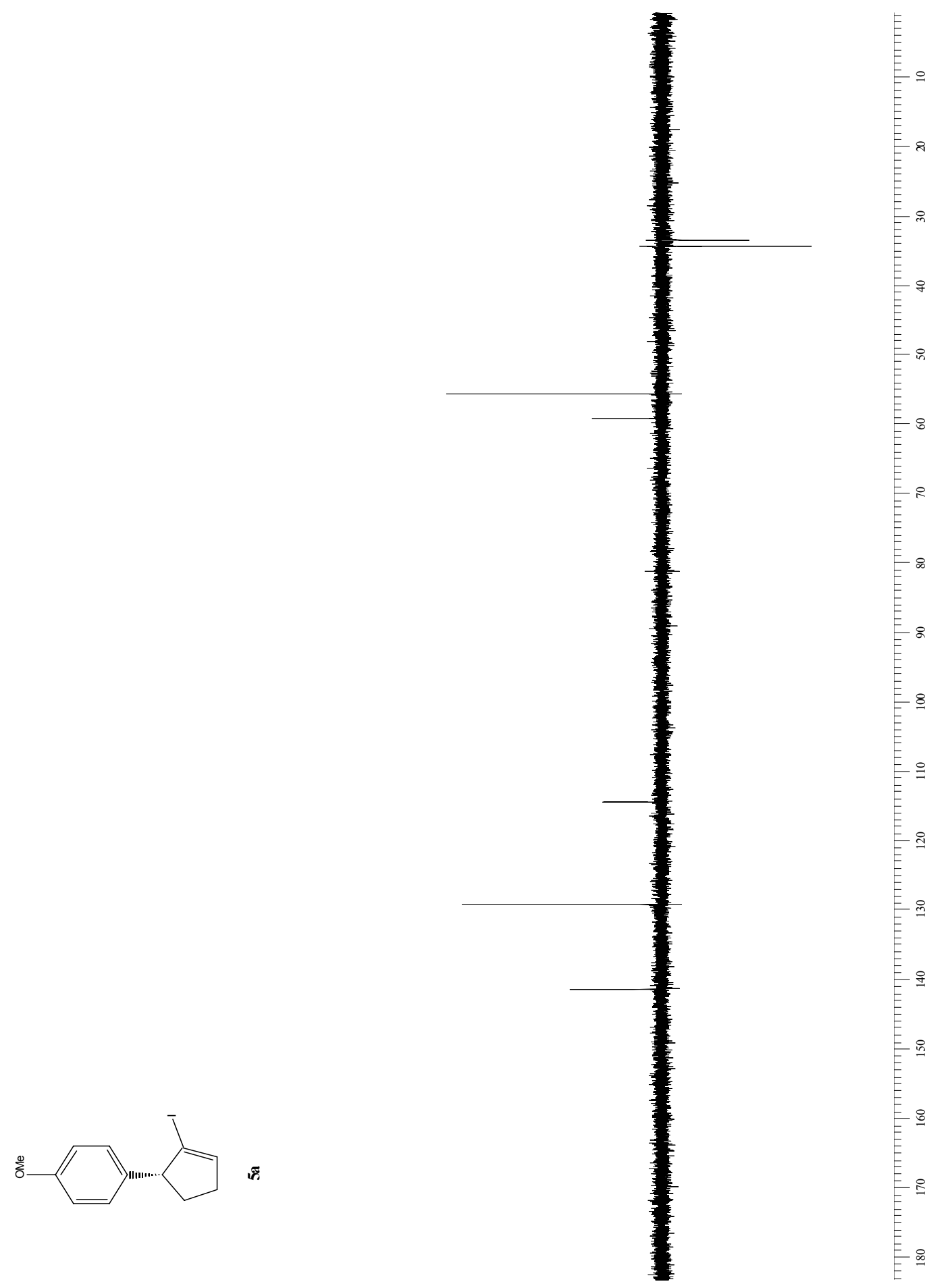




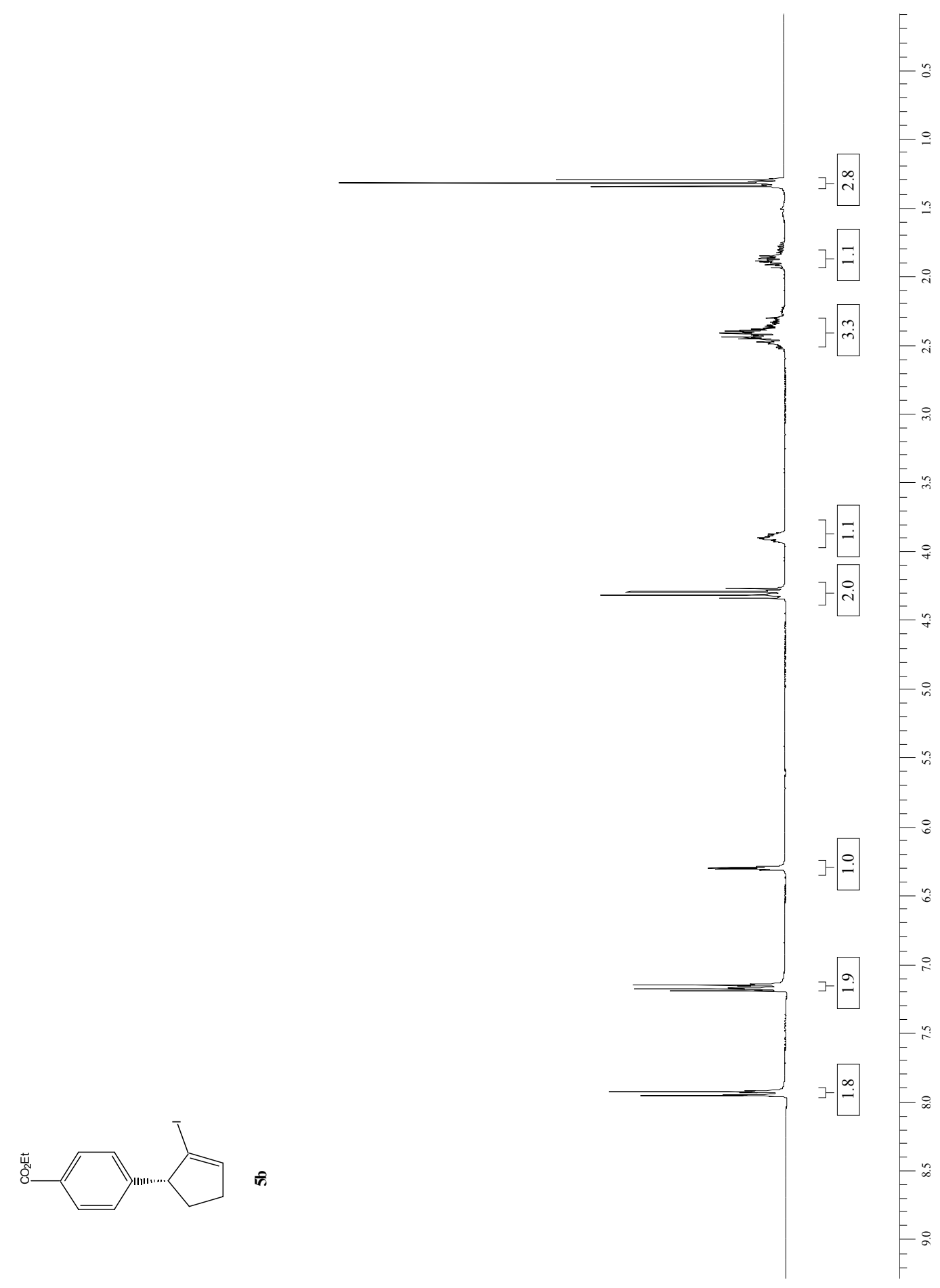




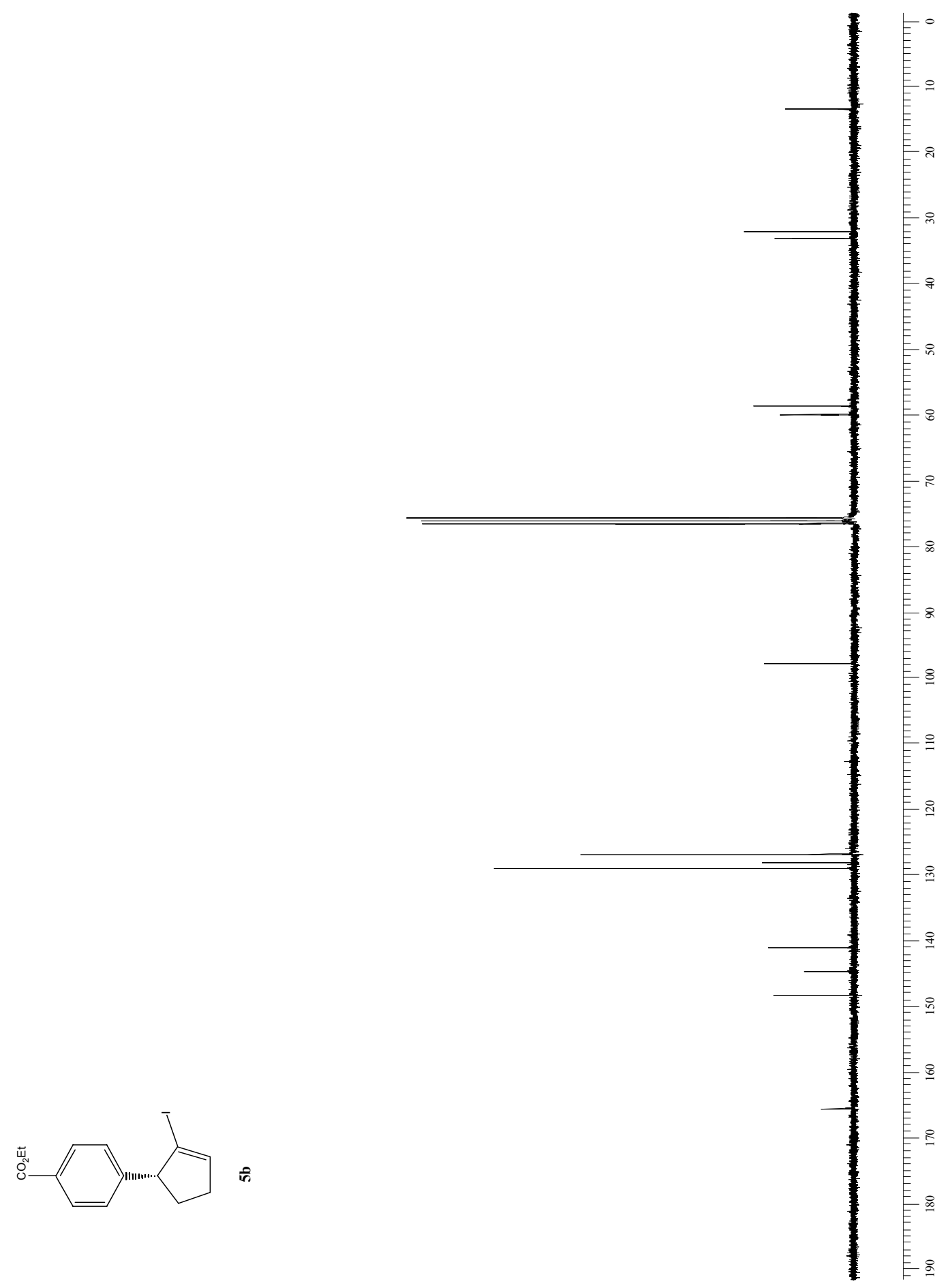




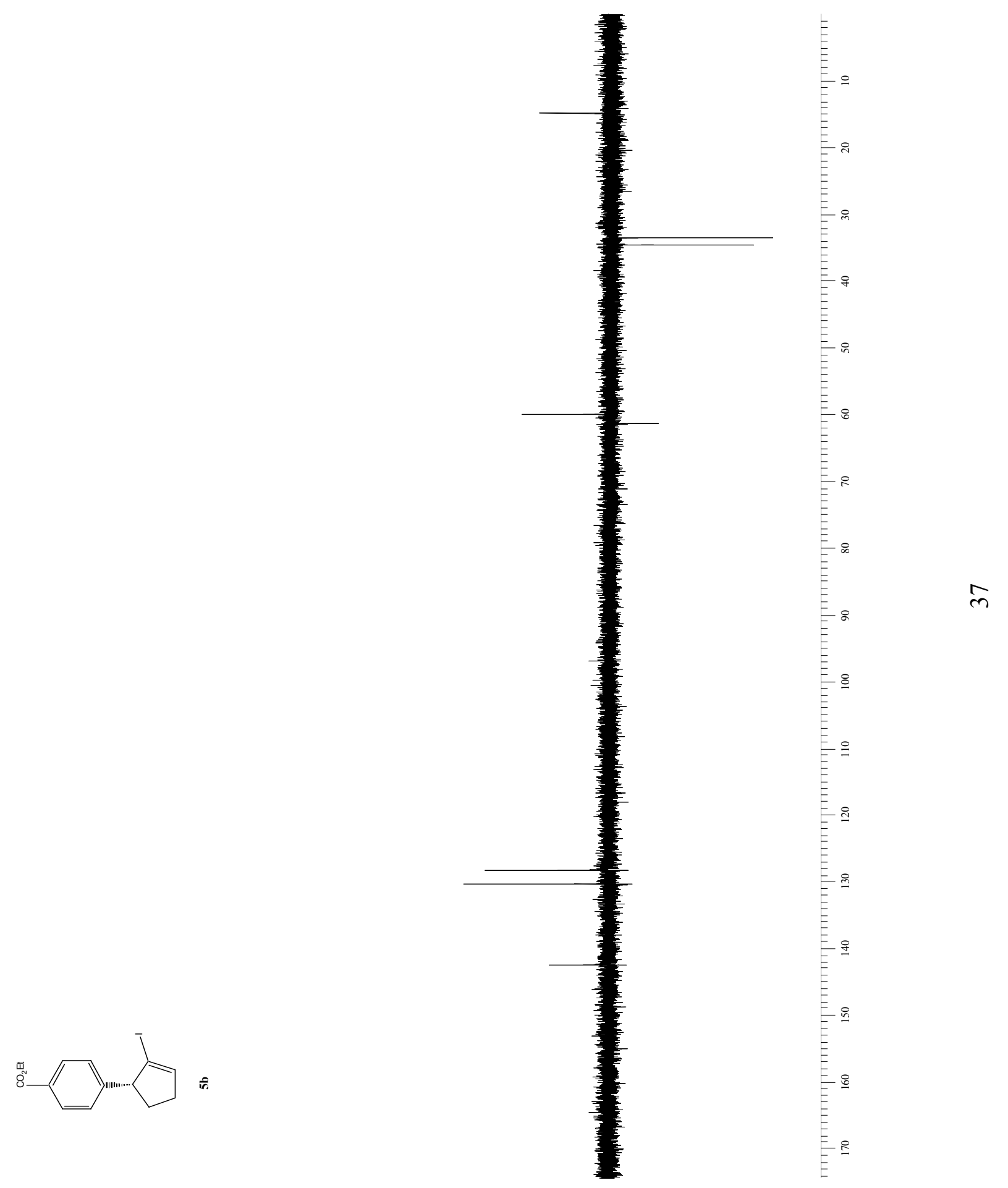




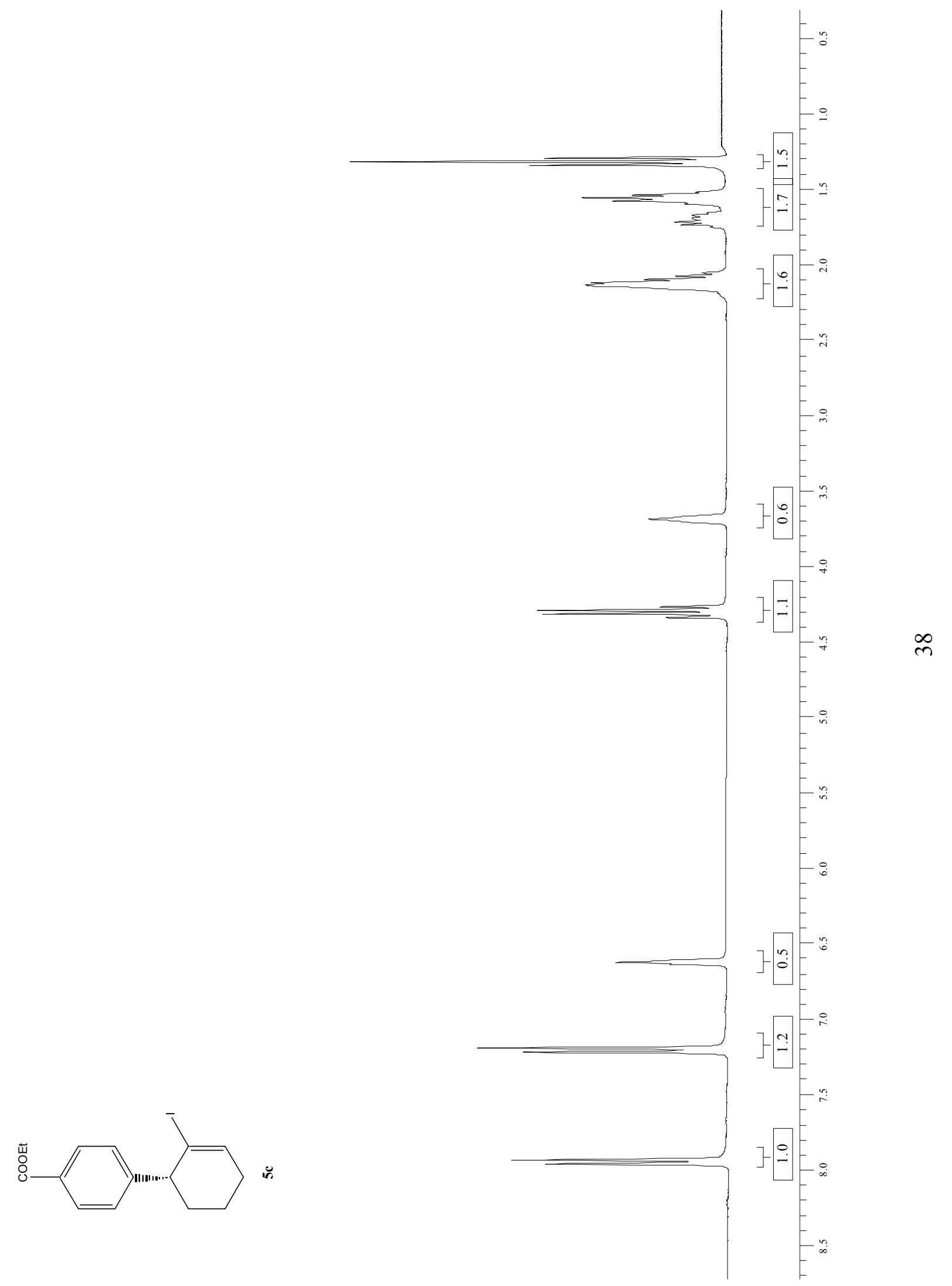




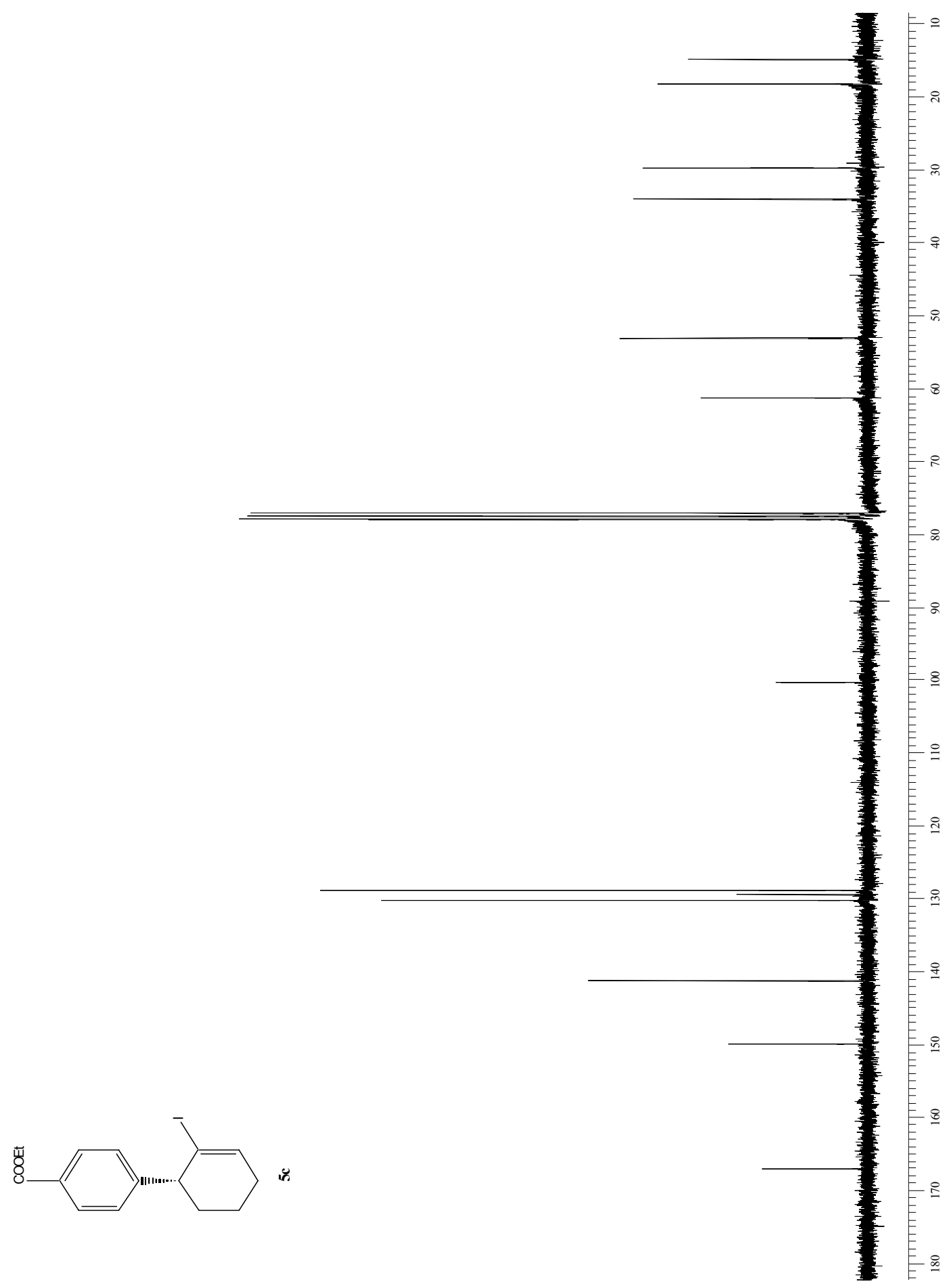


<smiles>CC(C)C</smiles> 


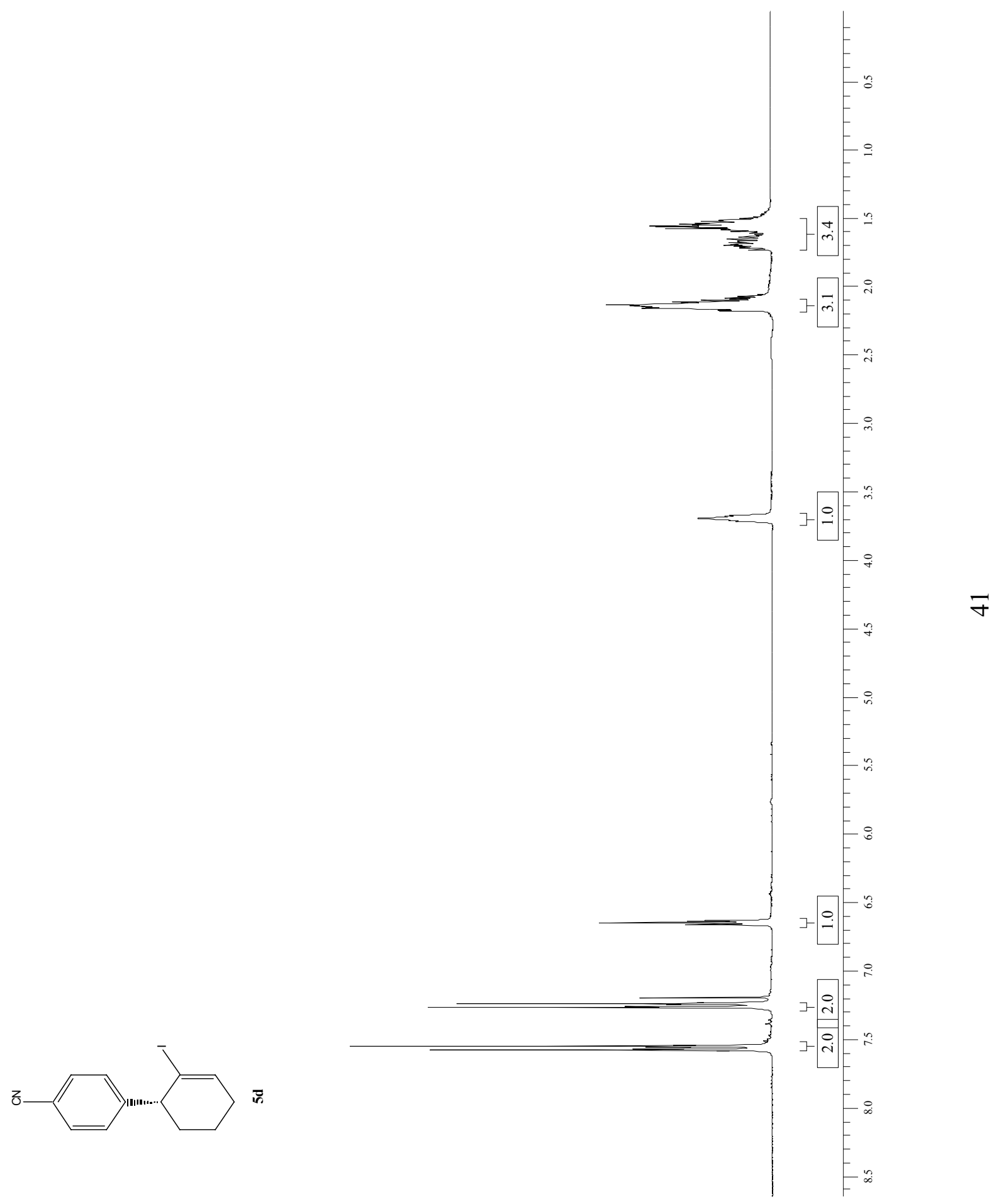




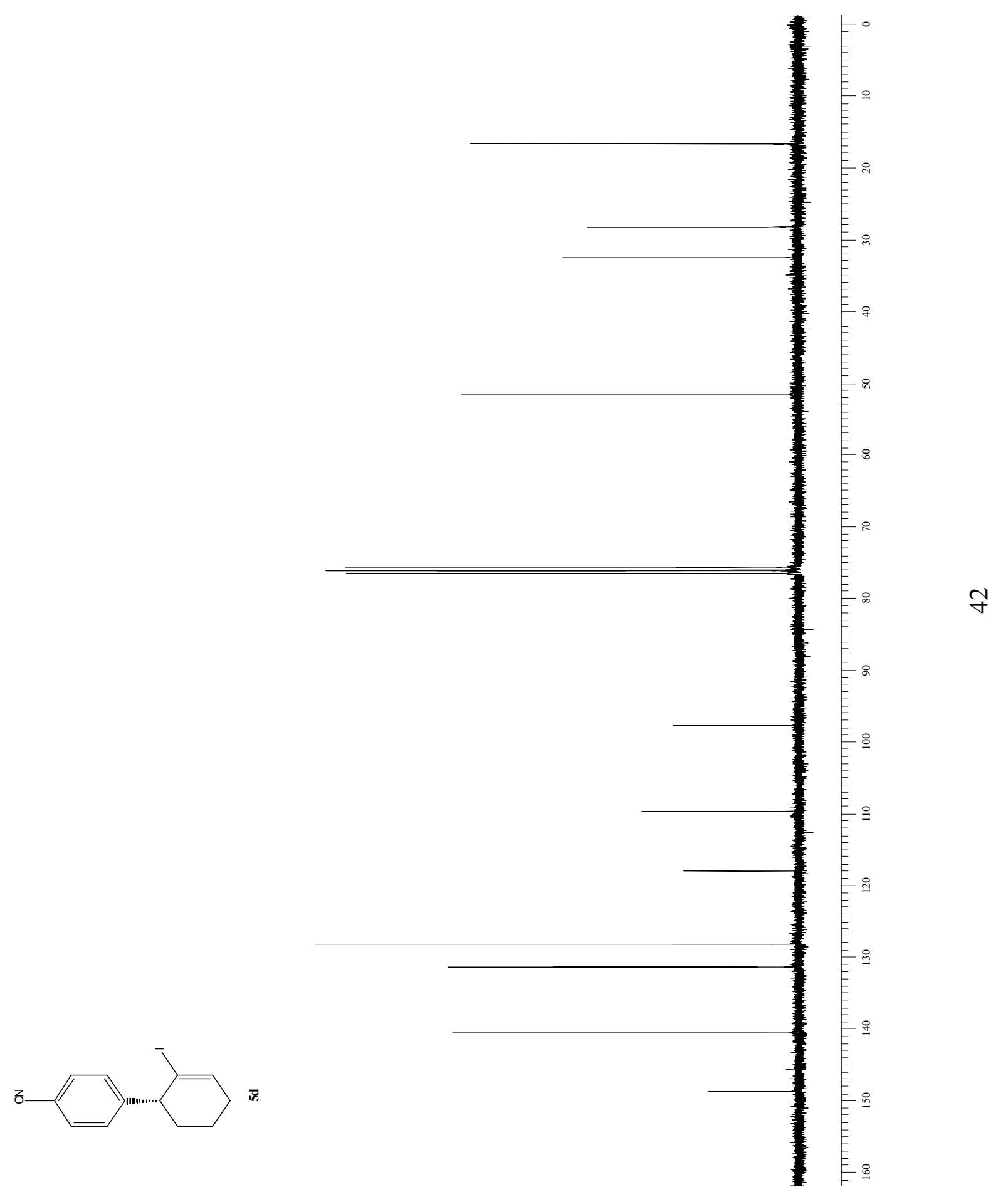




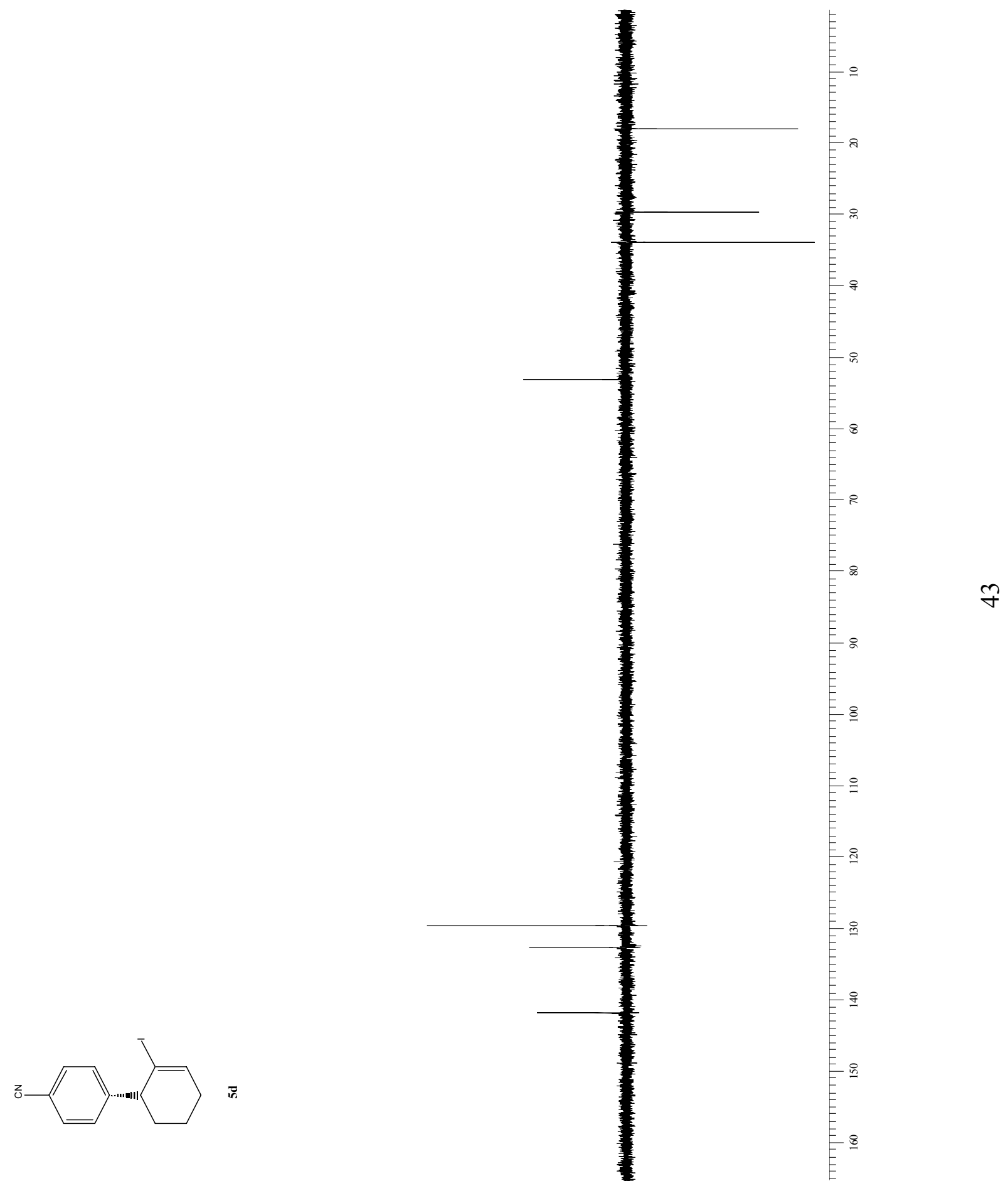




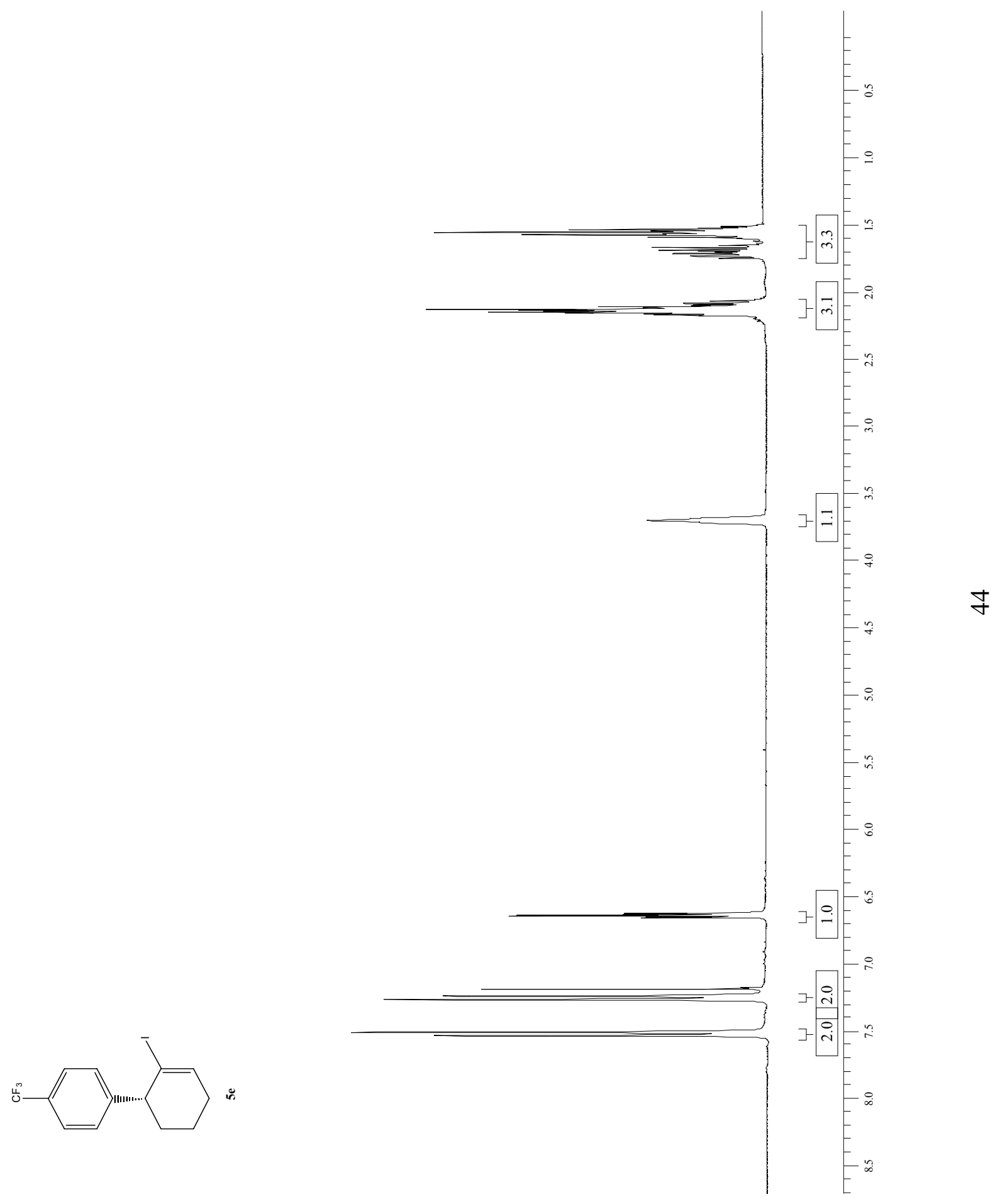




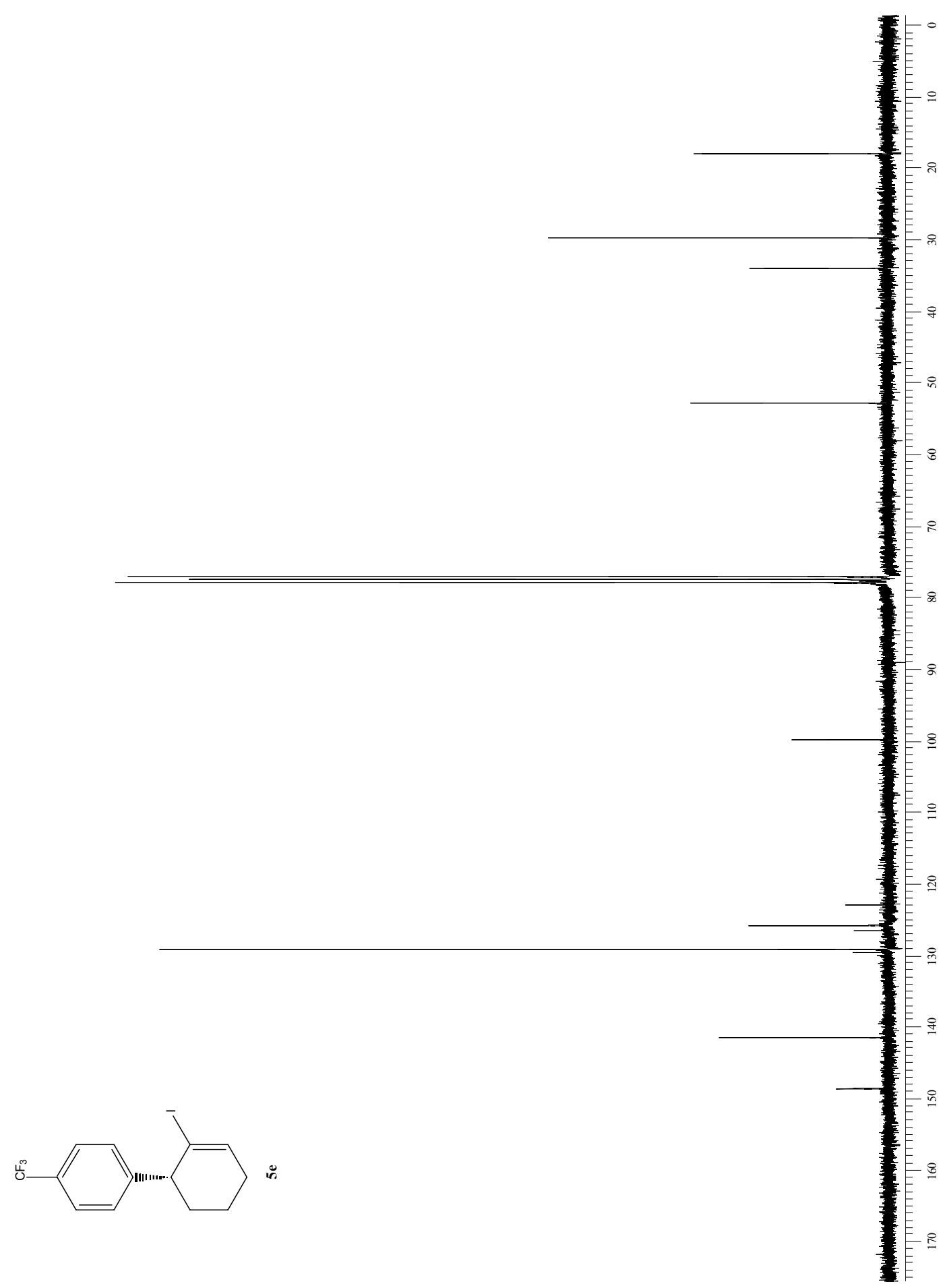




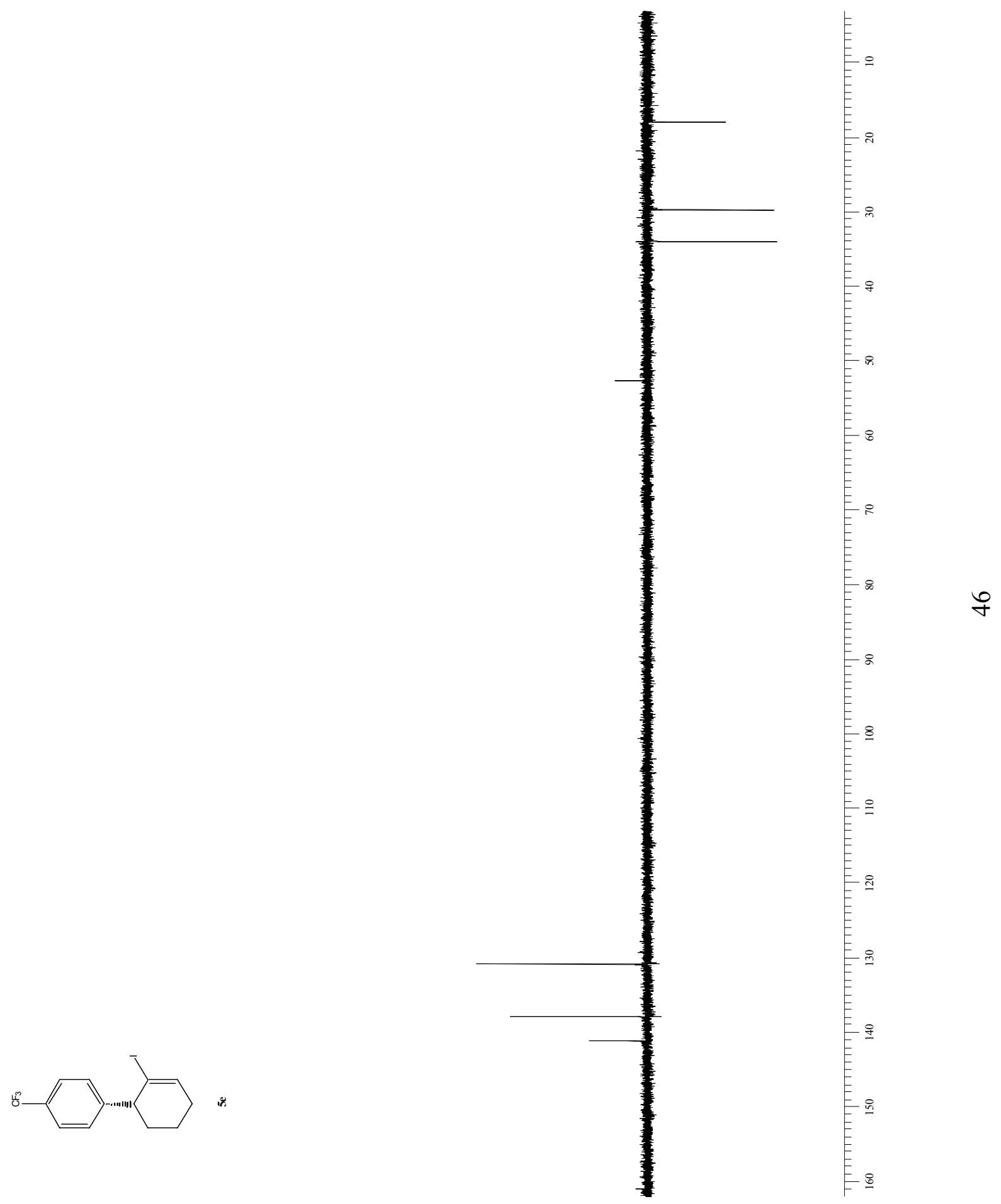




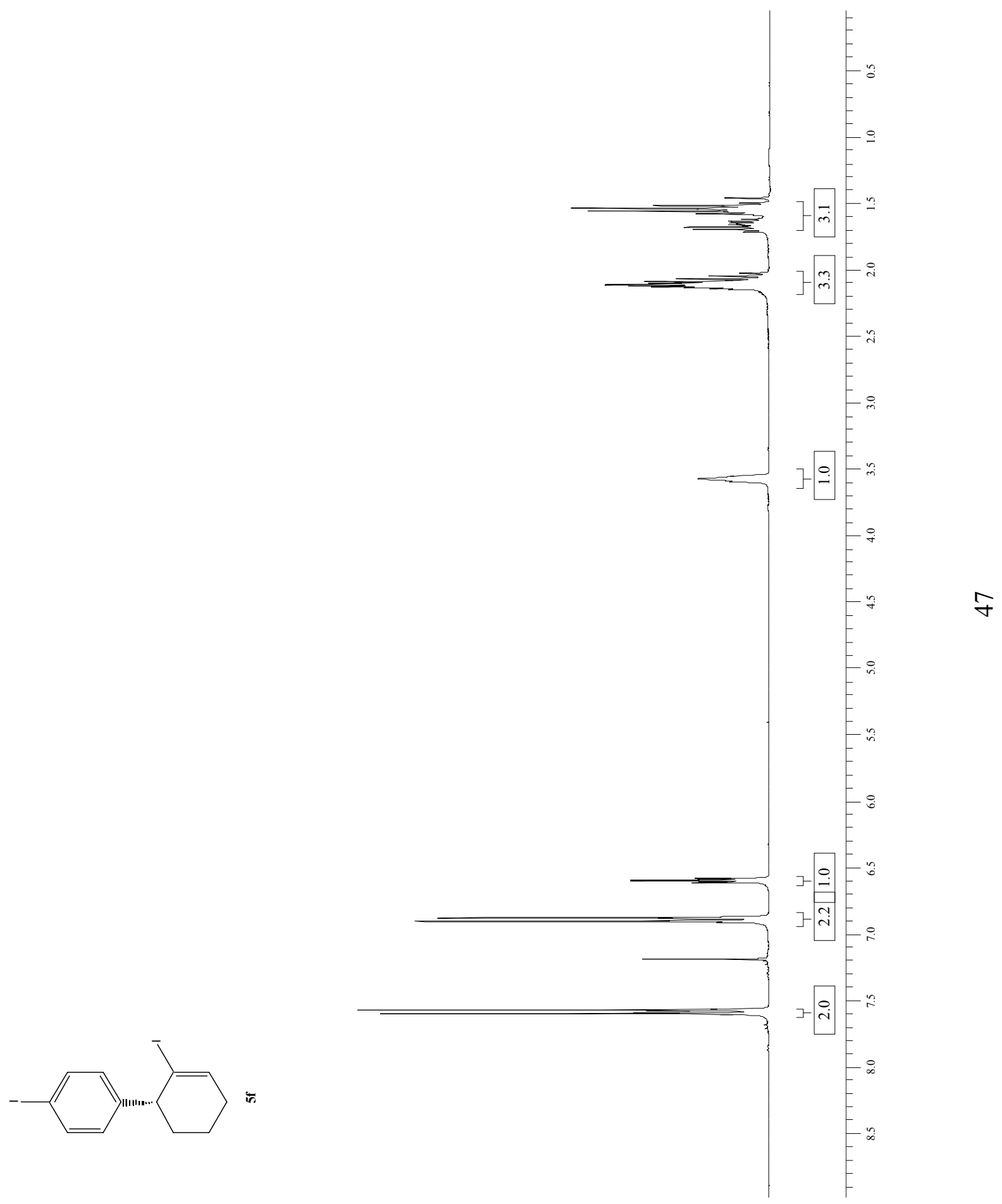




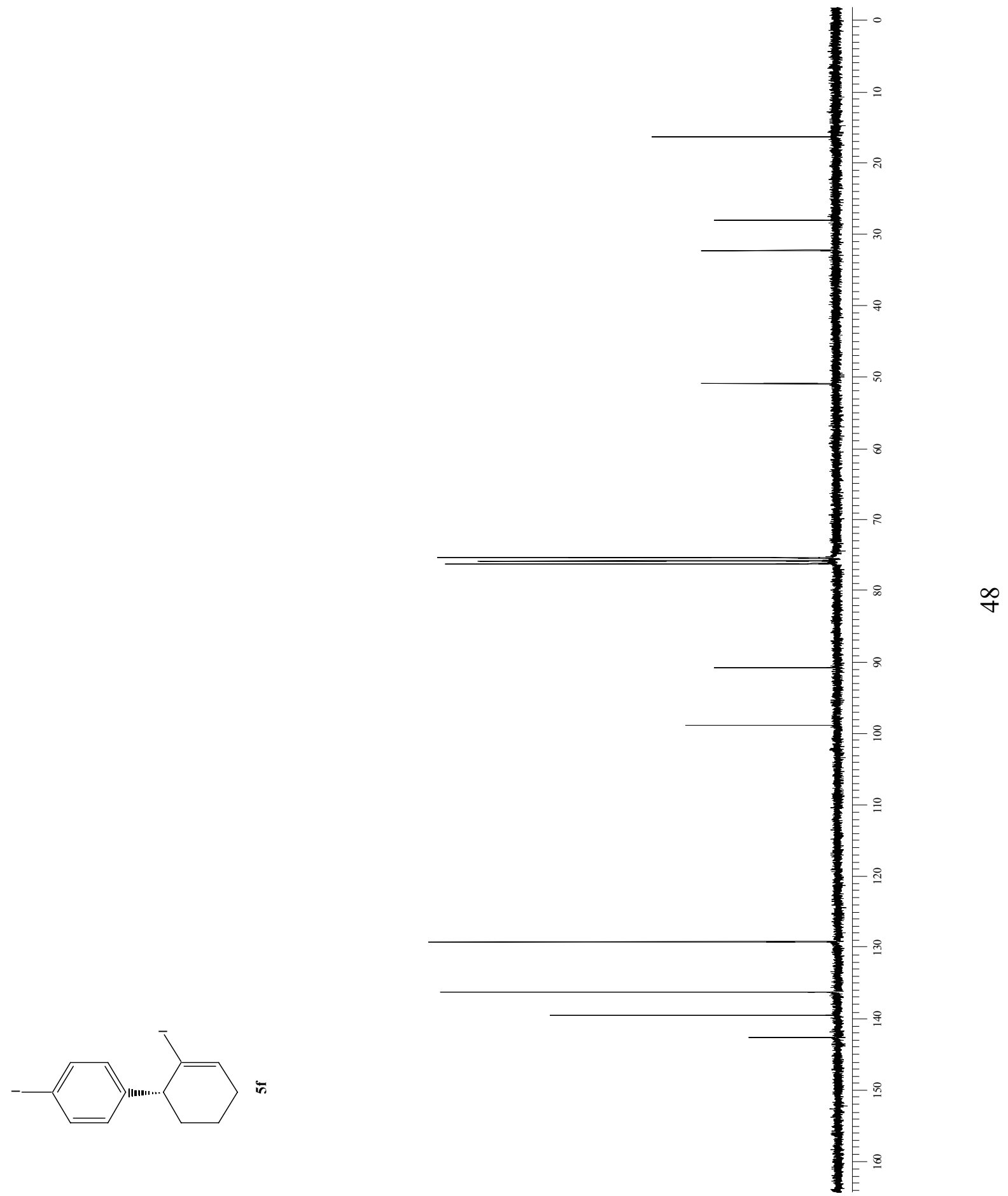




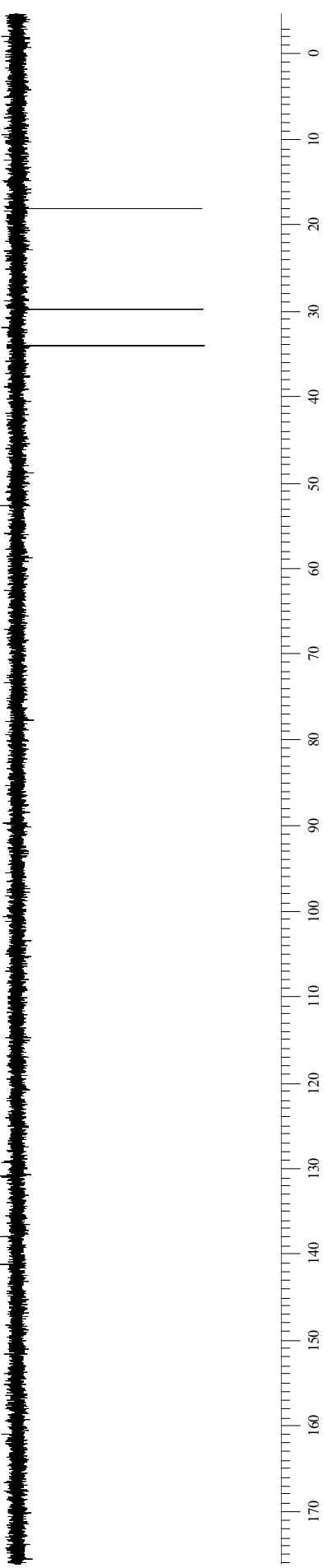




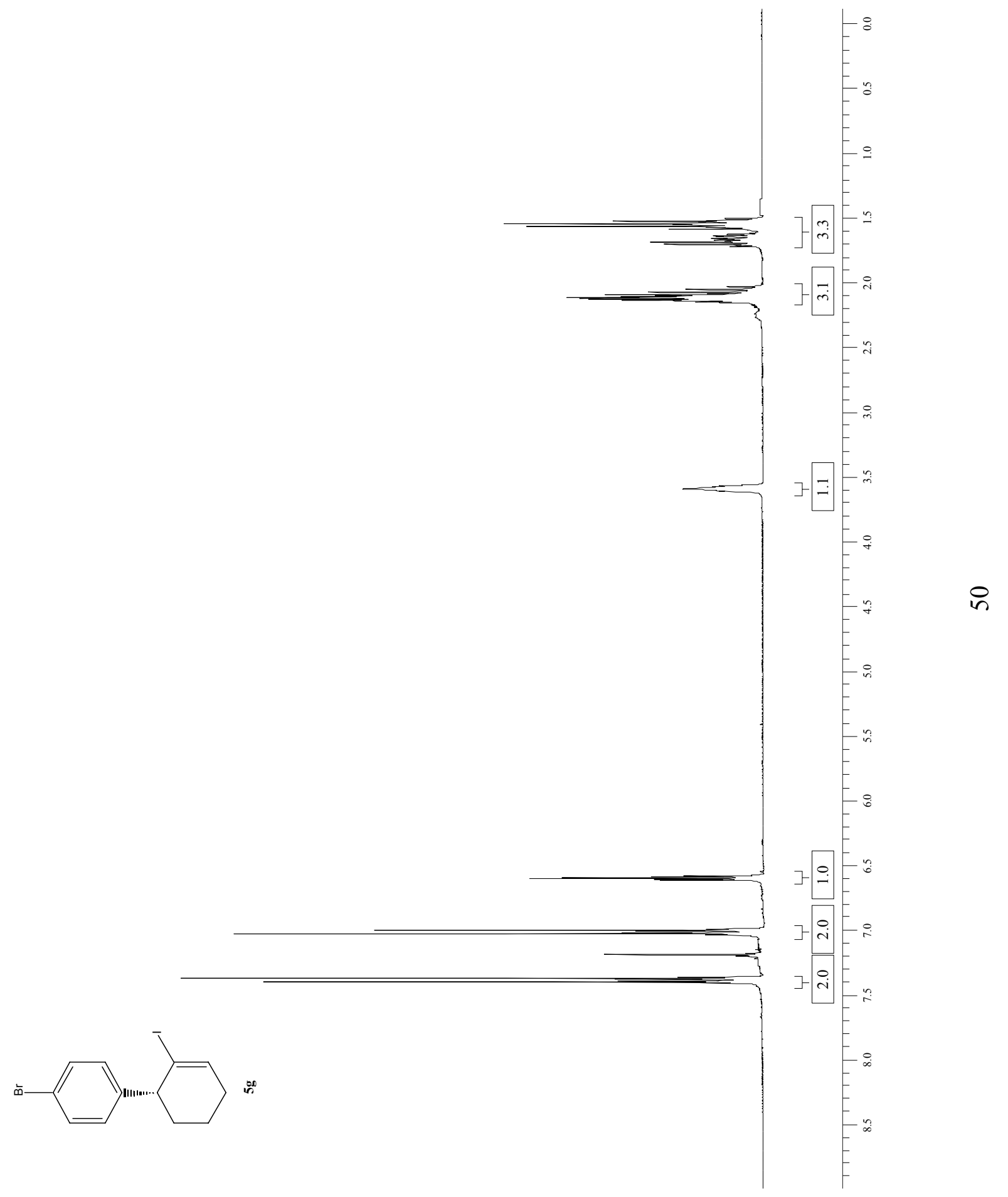




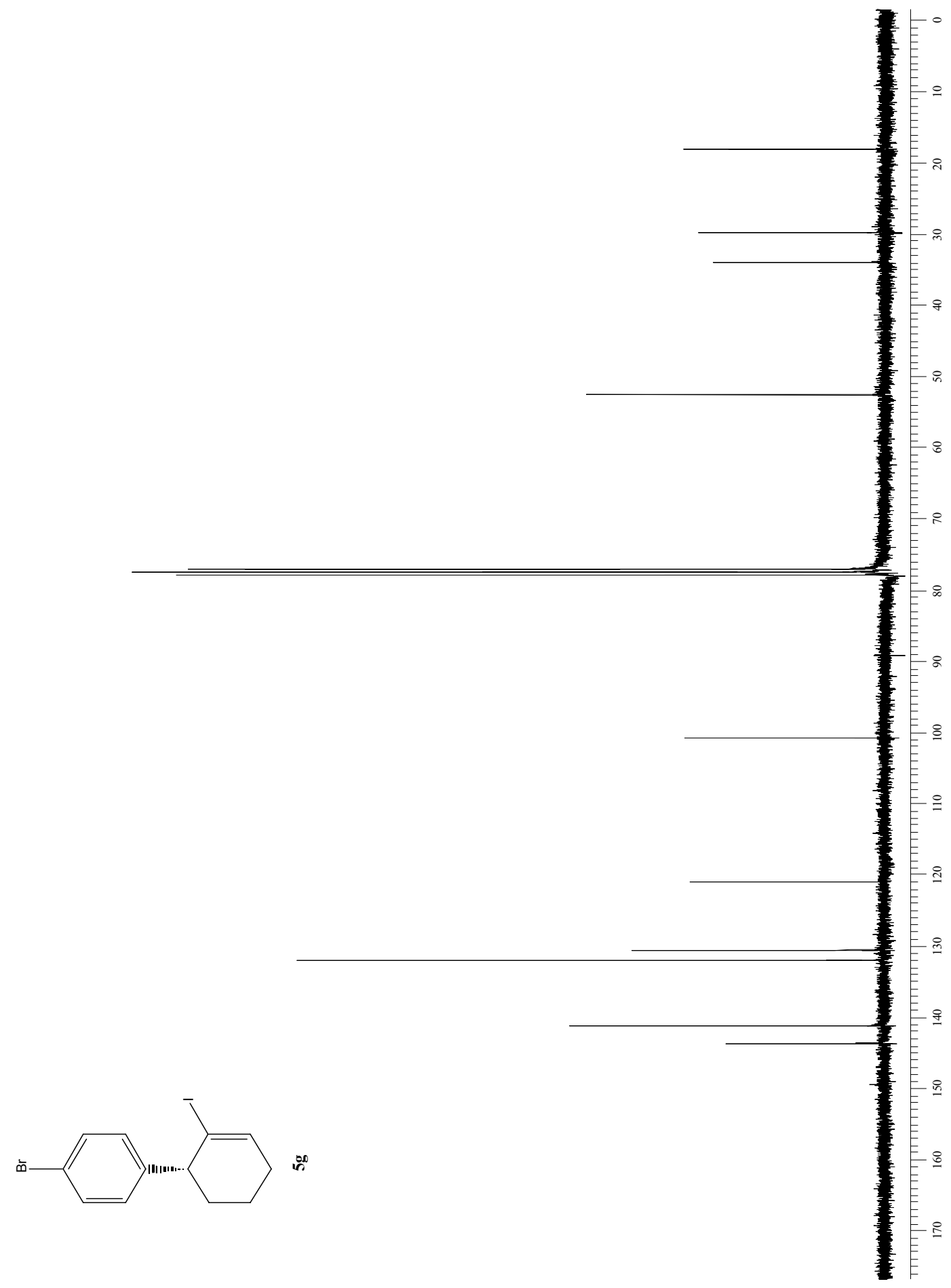


n

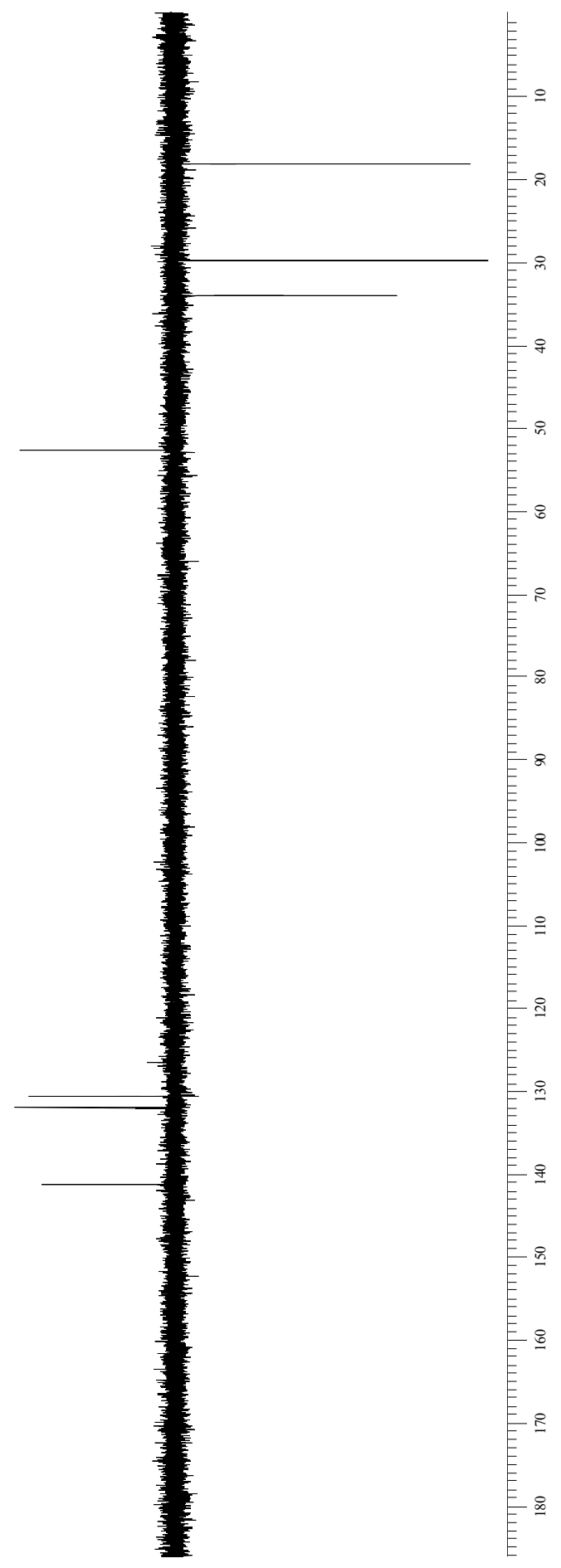




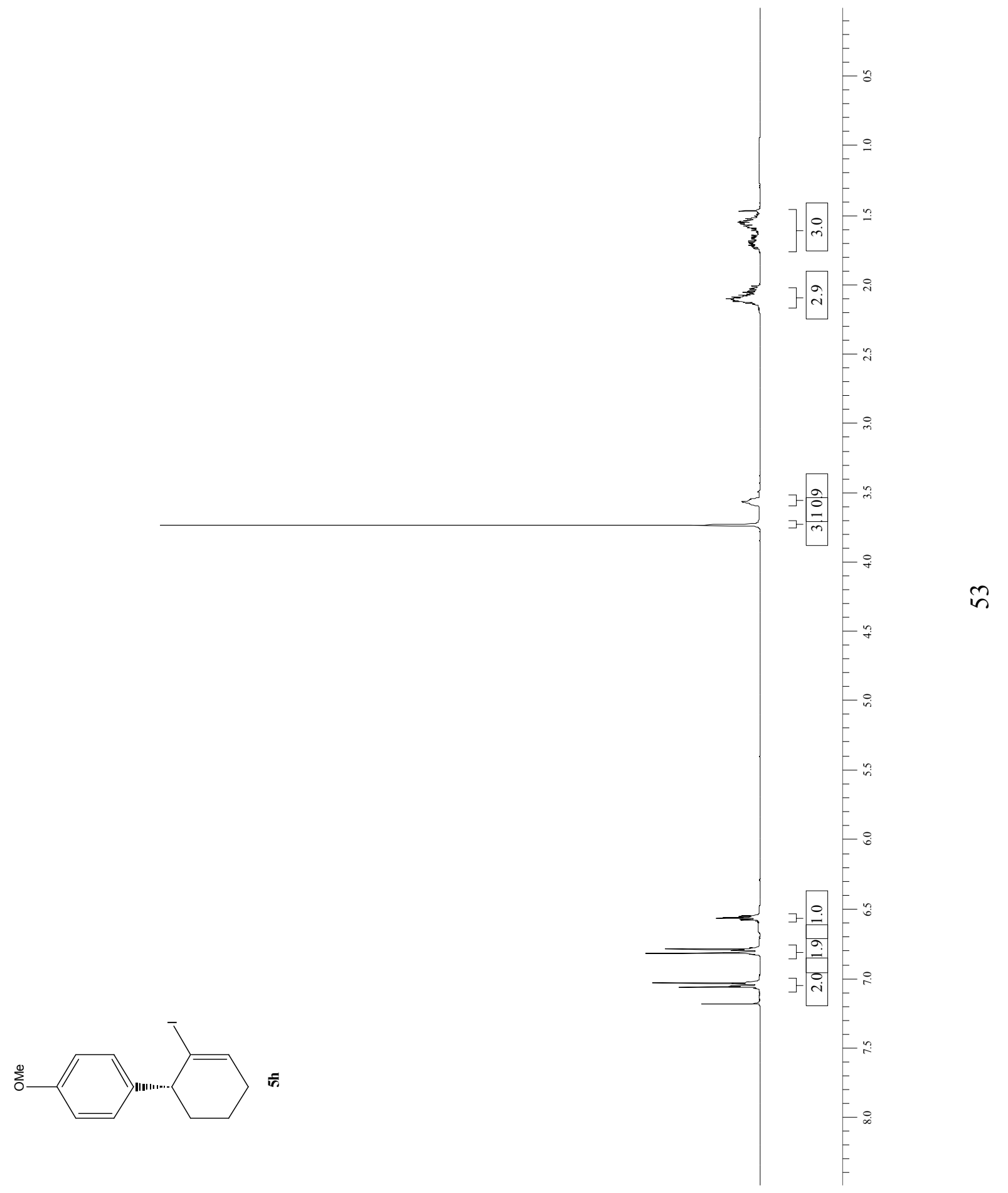




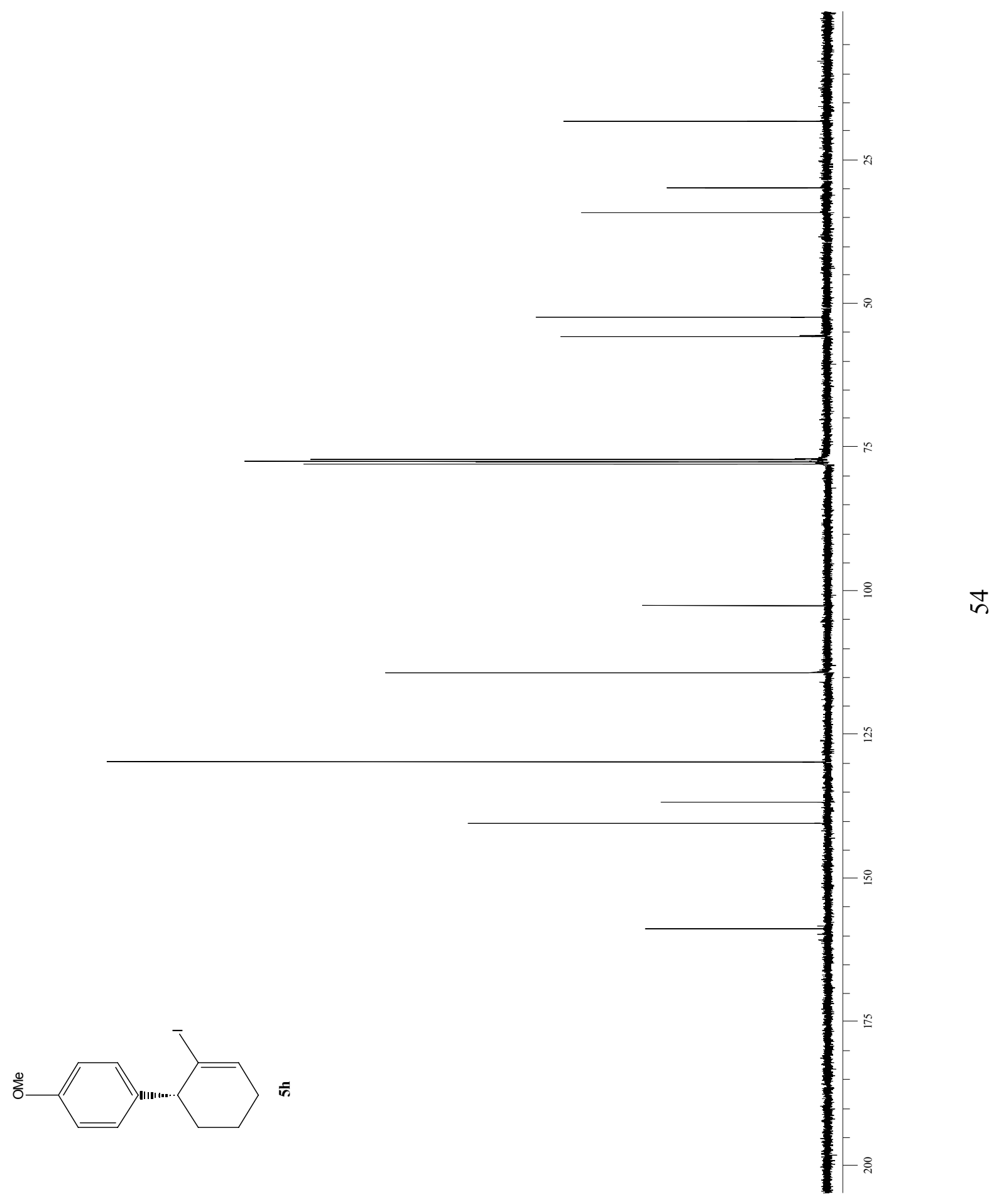


n

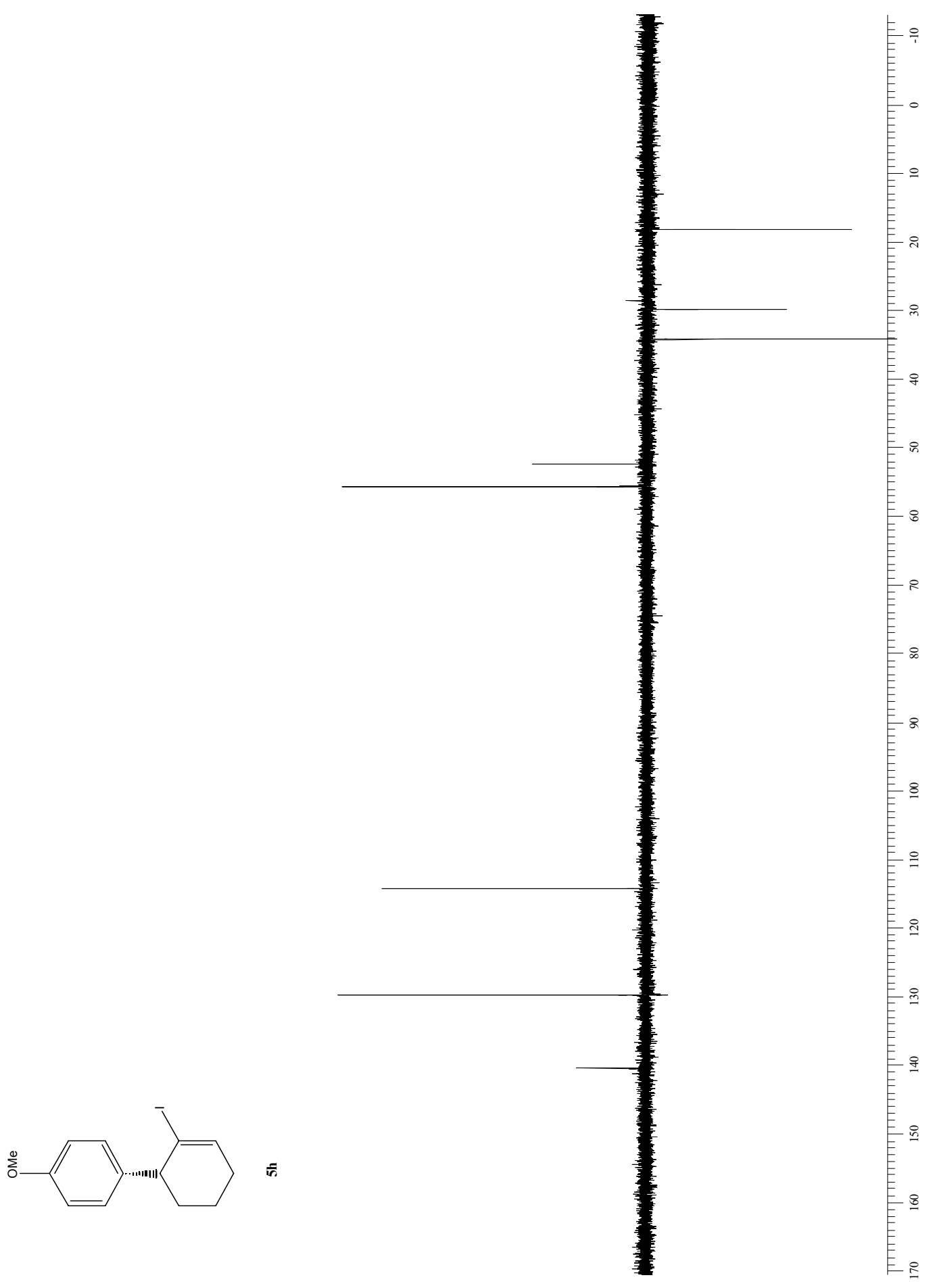




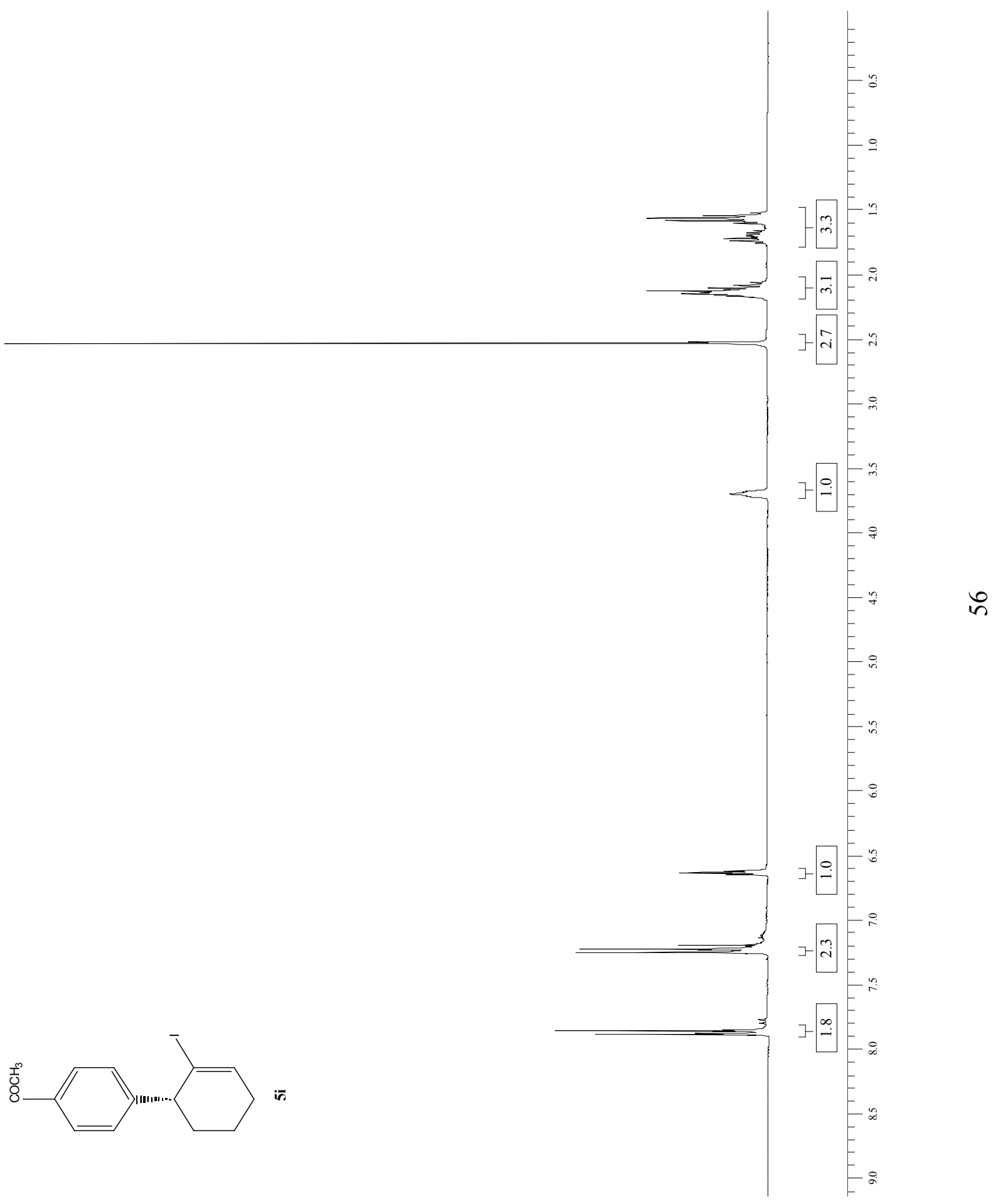


in

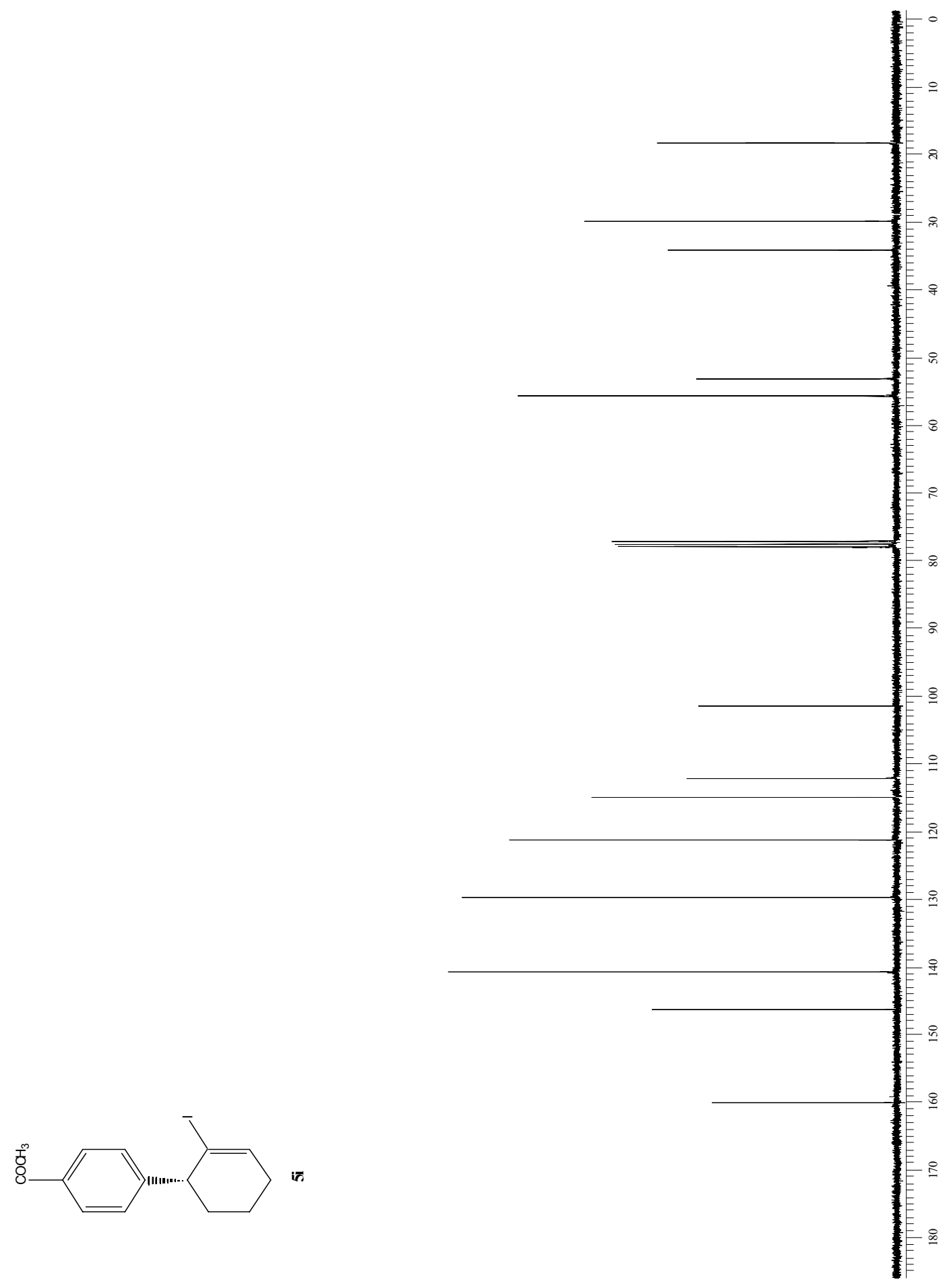




$$
\text { n }
$$

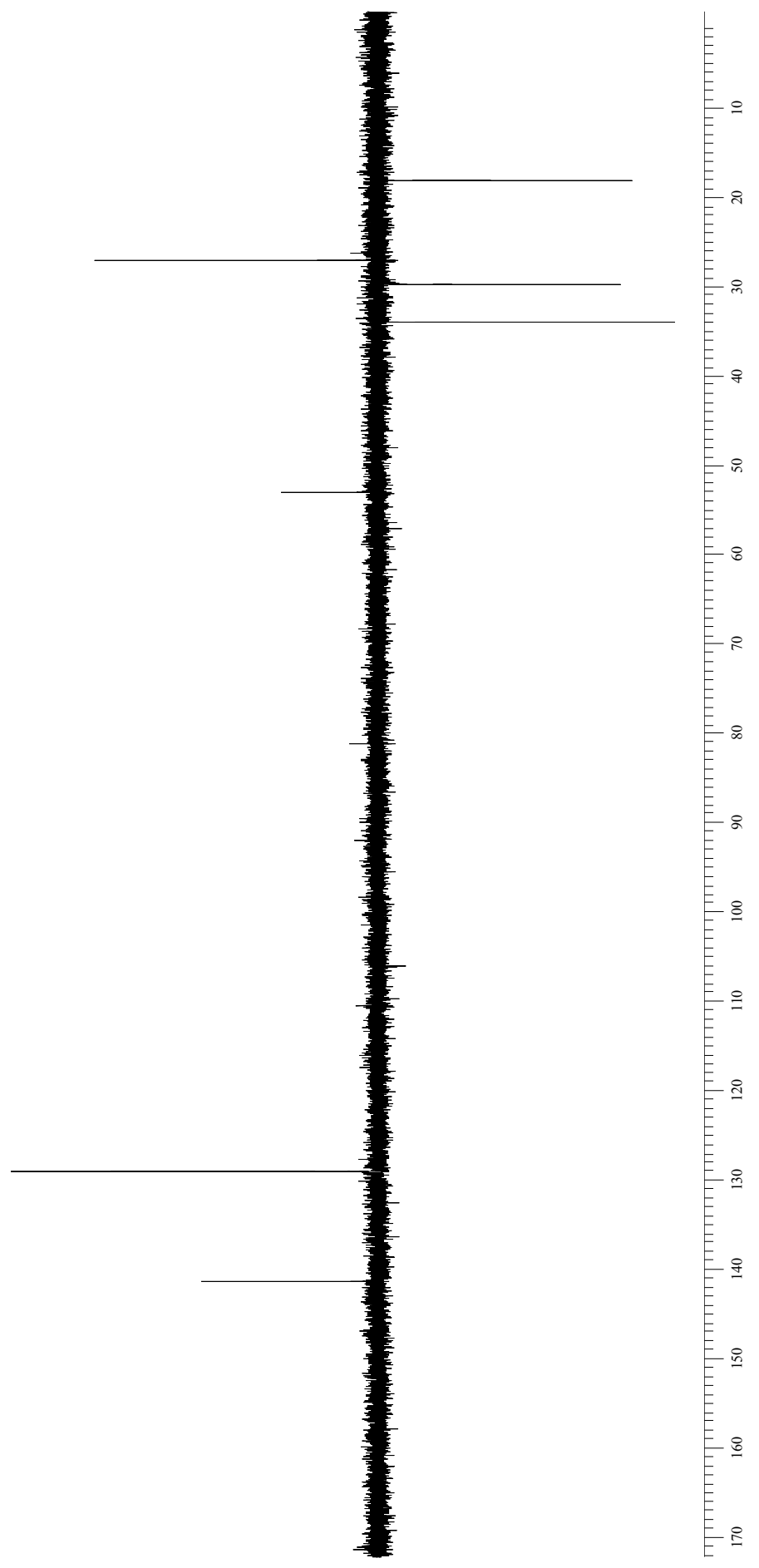




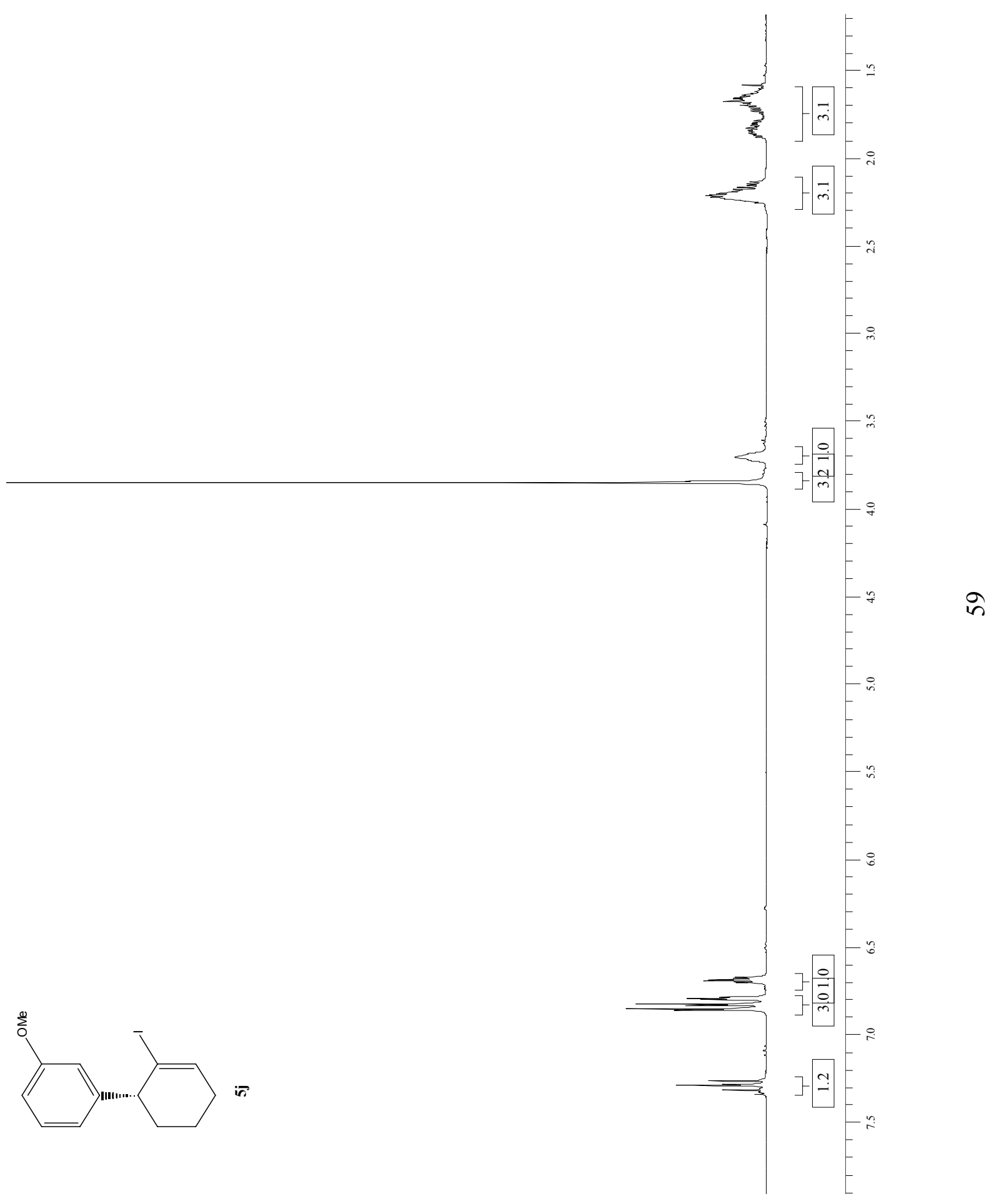




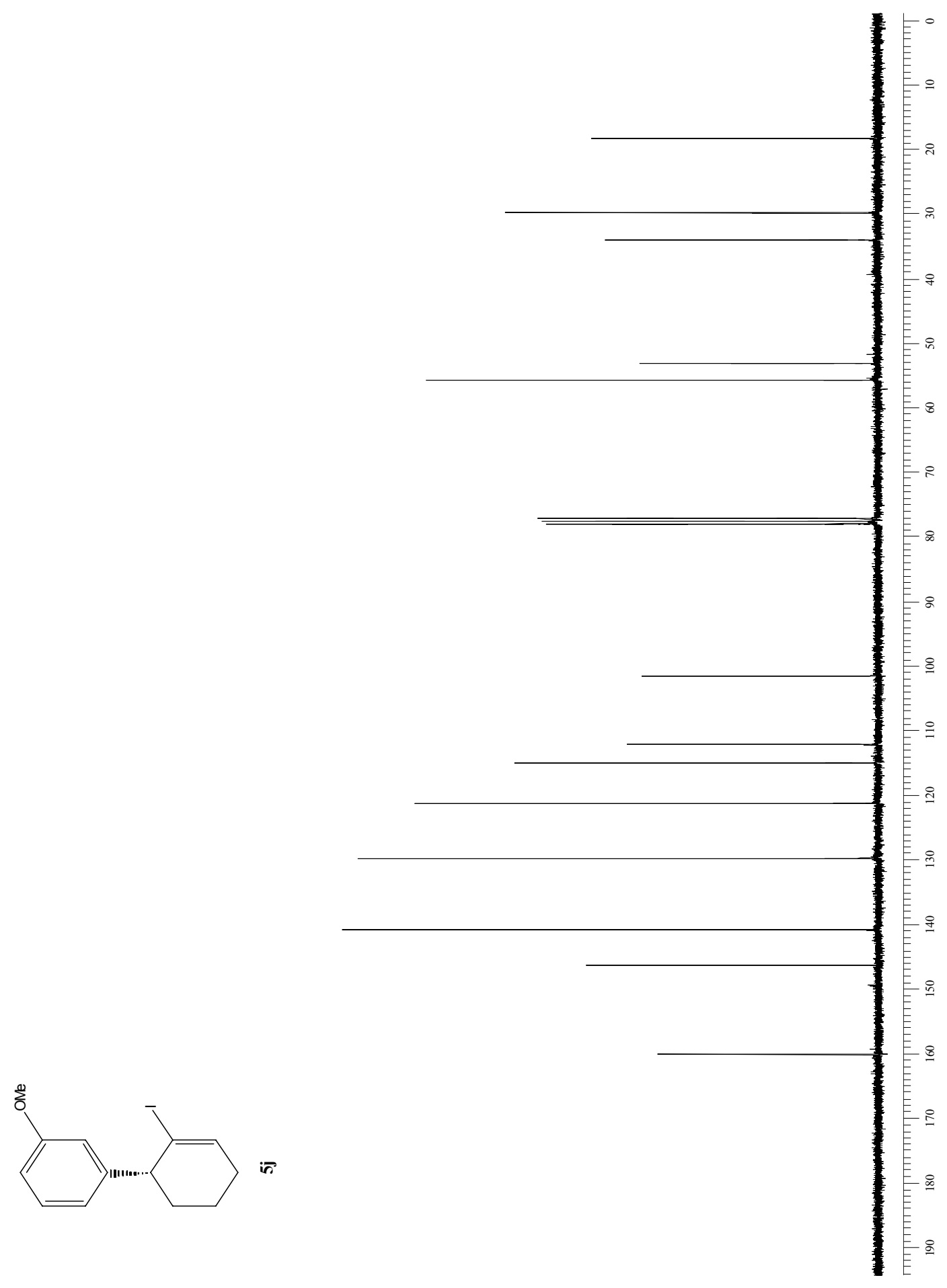


5
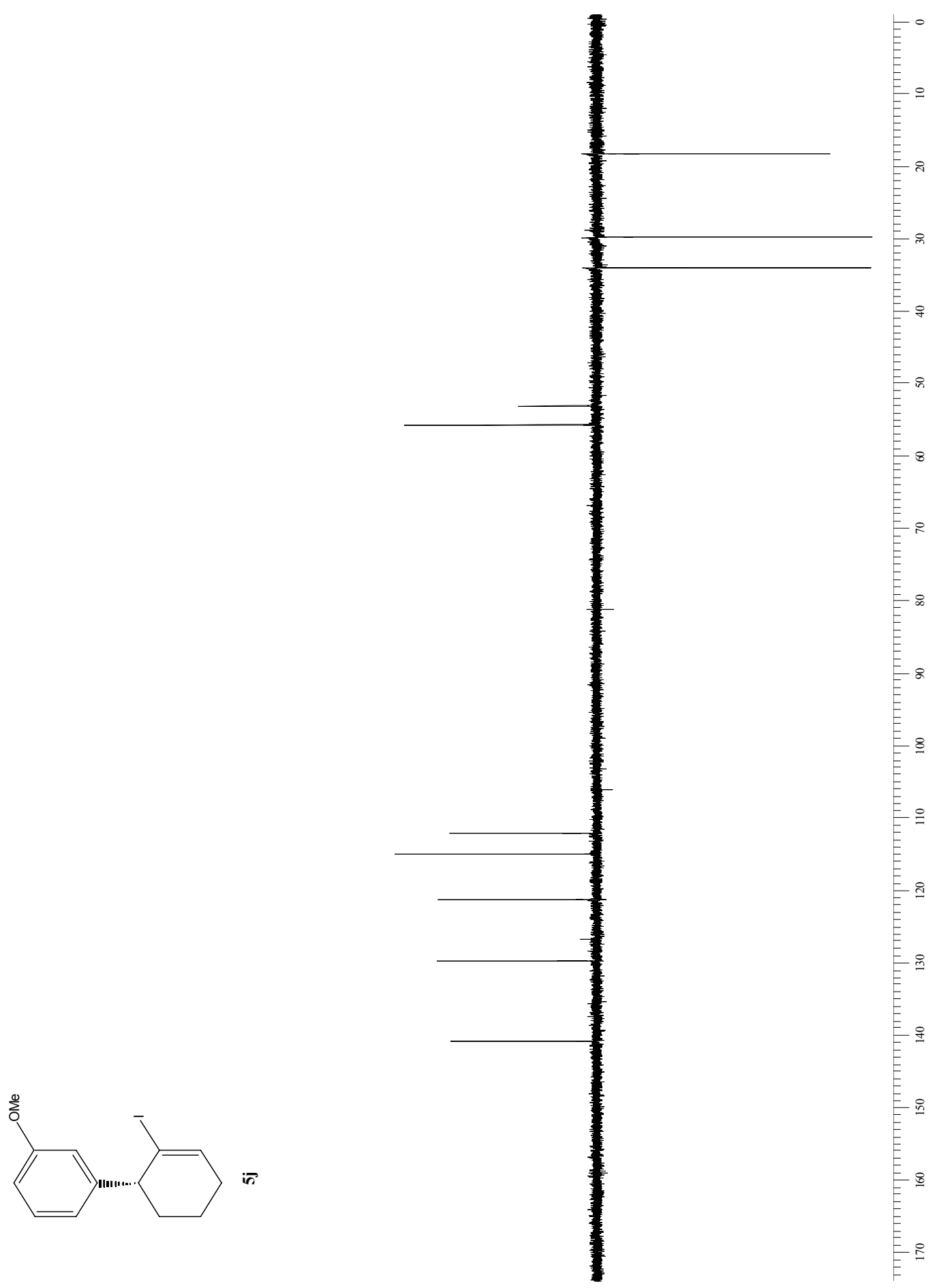


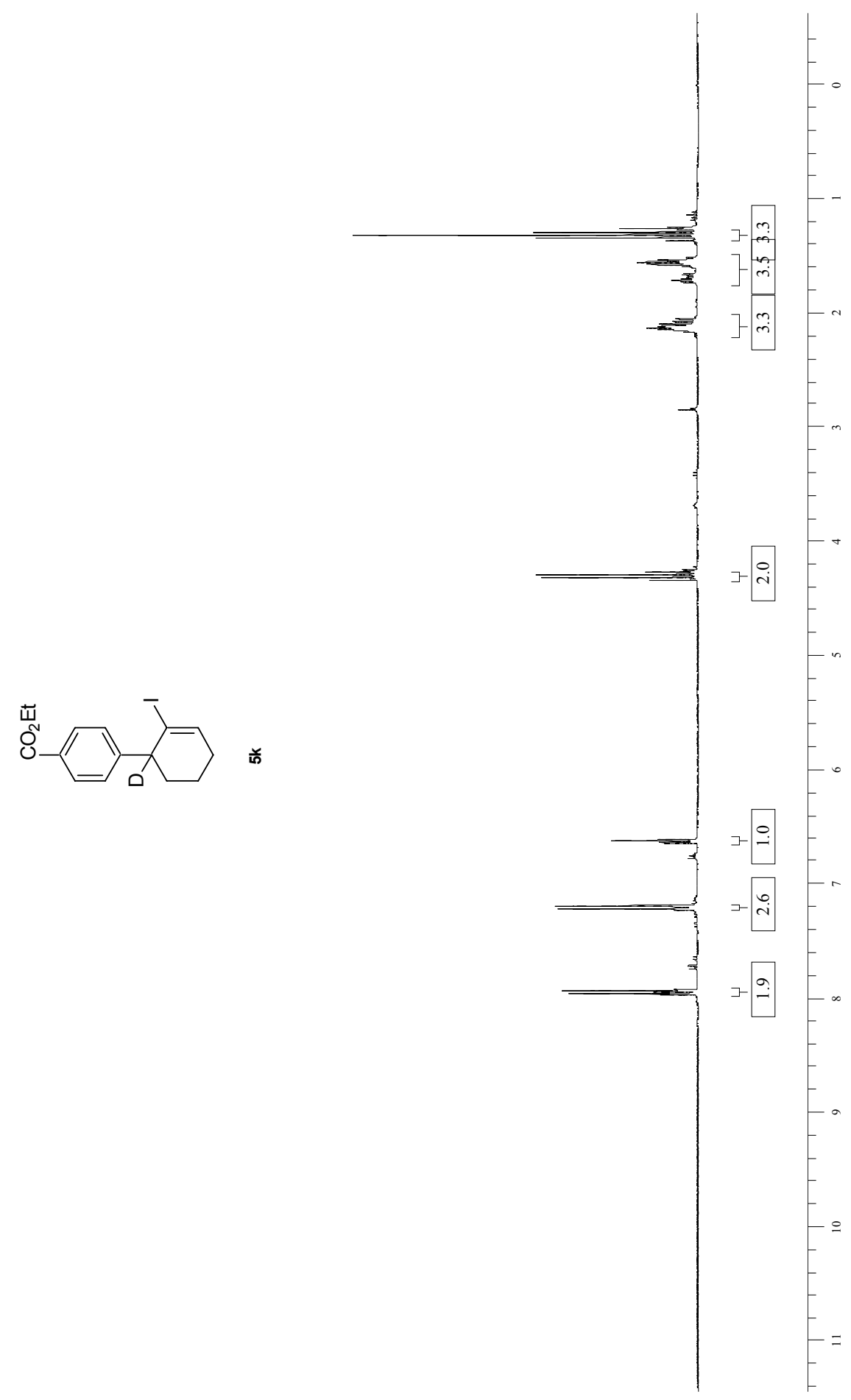




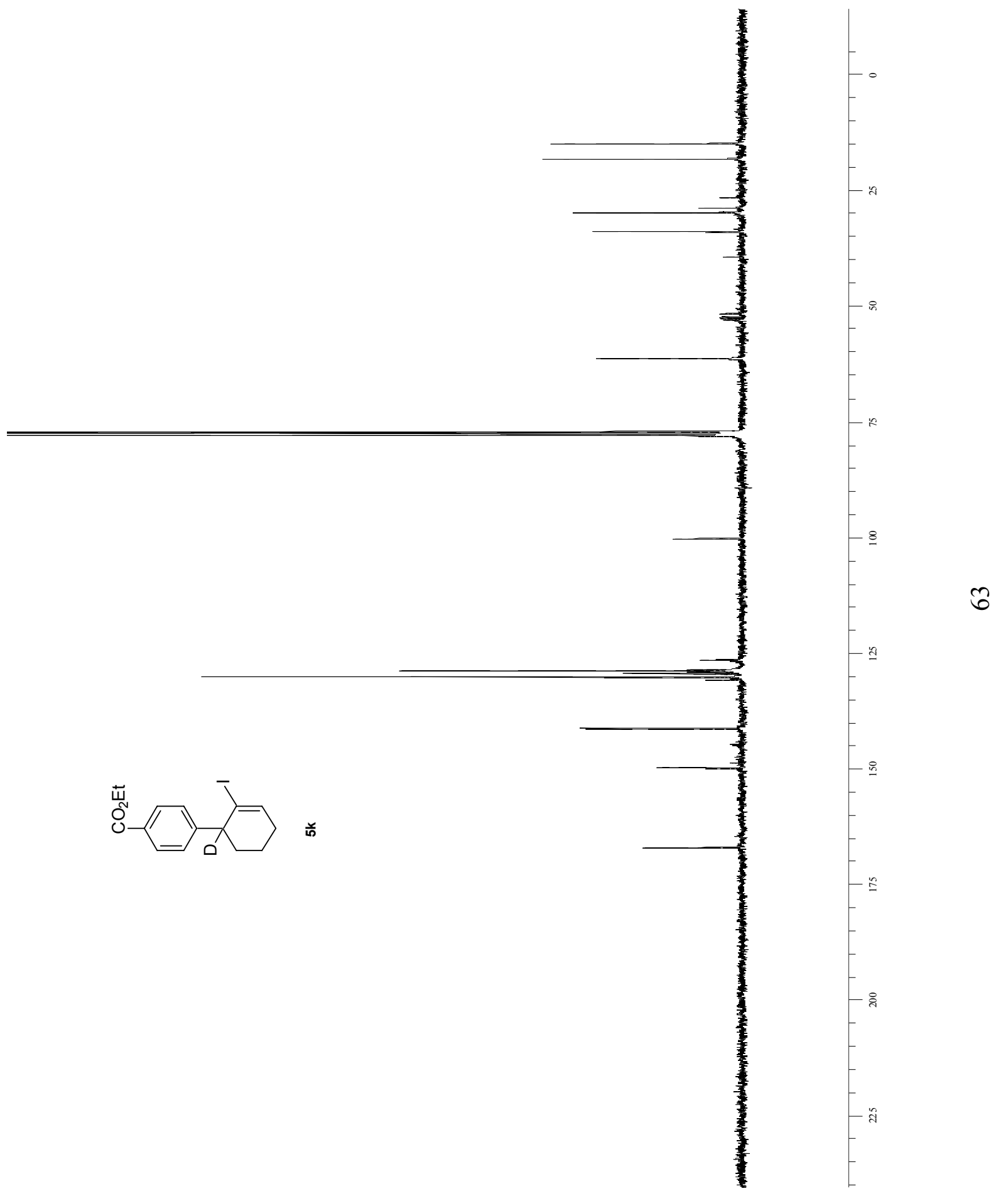




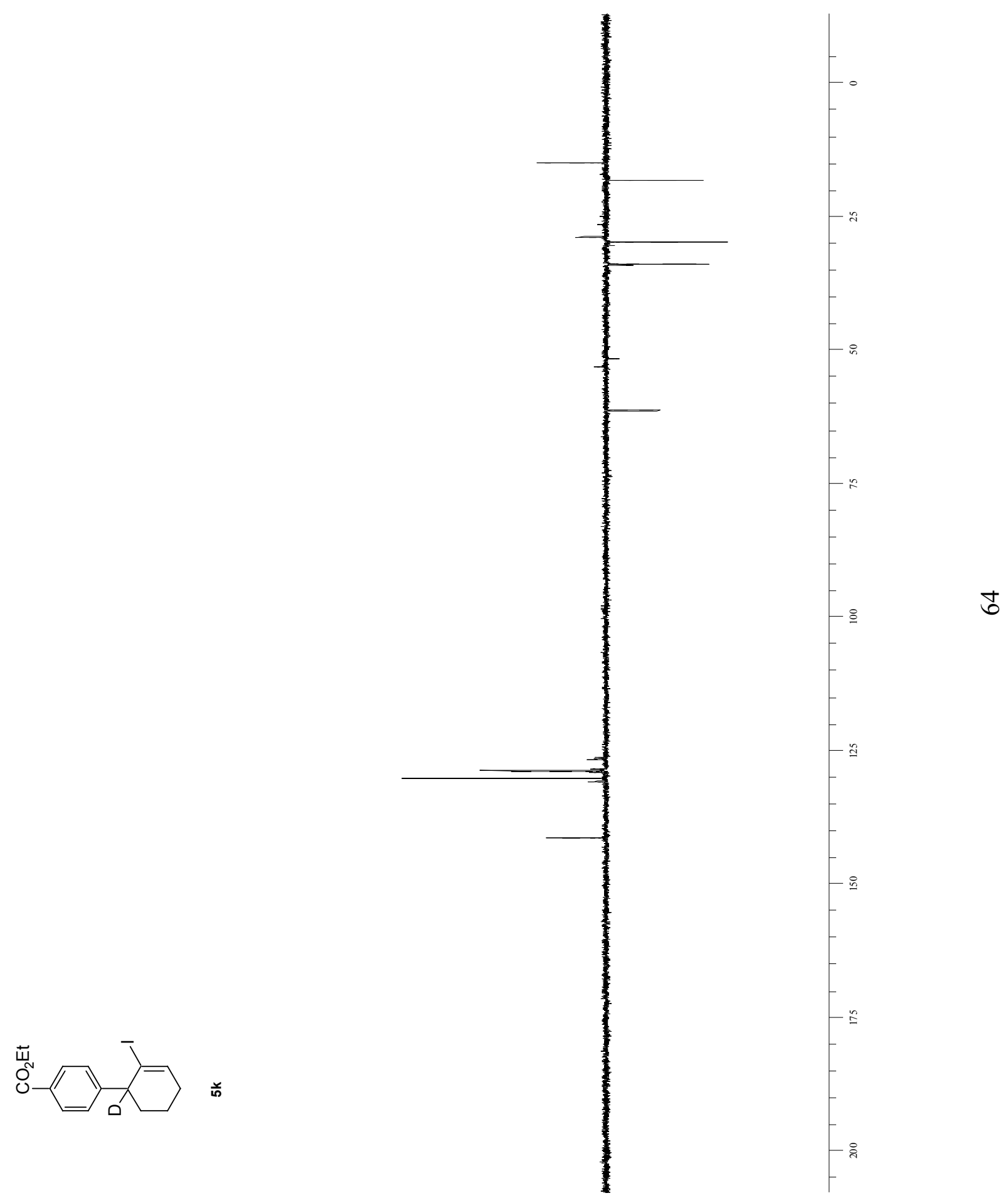




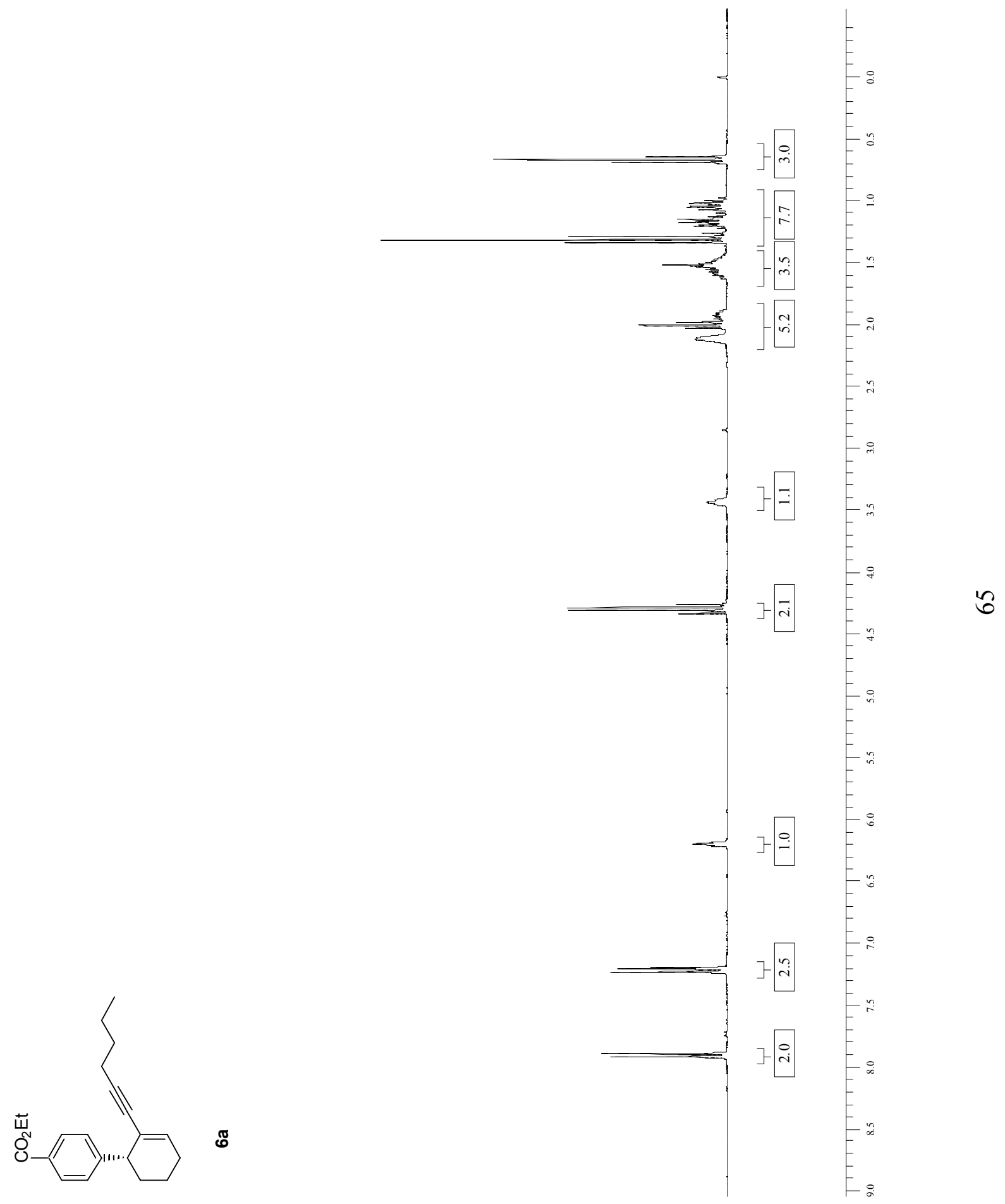




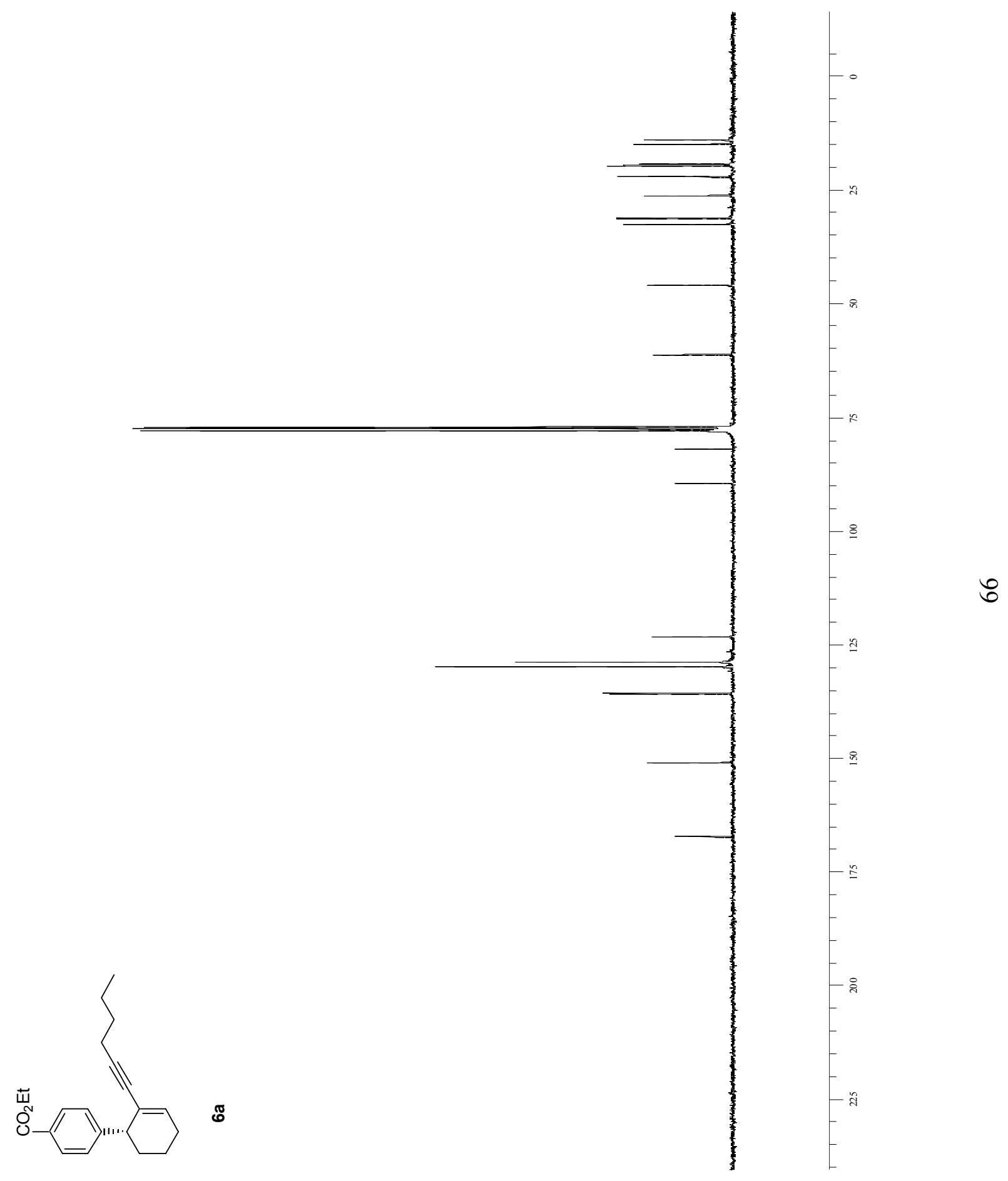




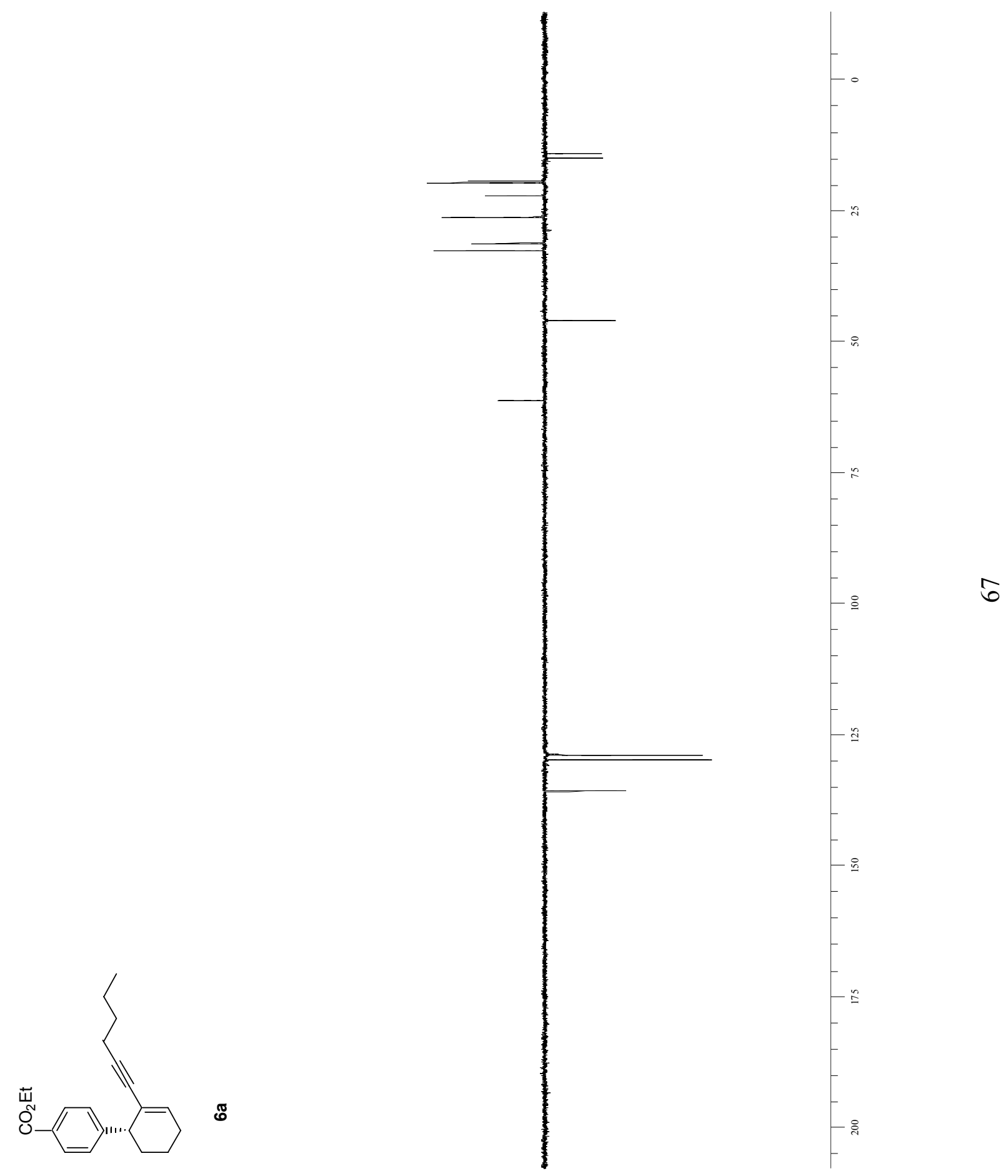




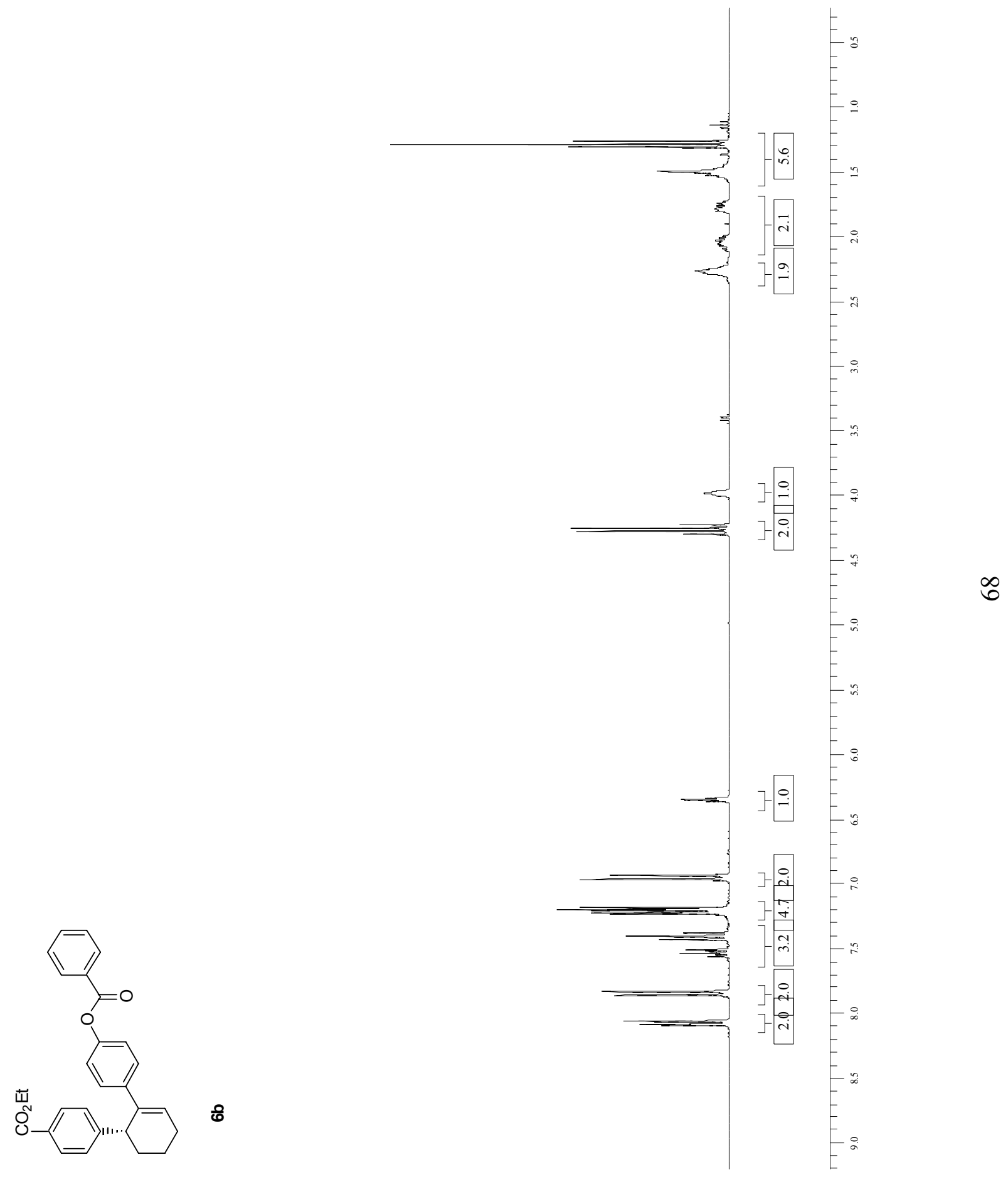


8)
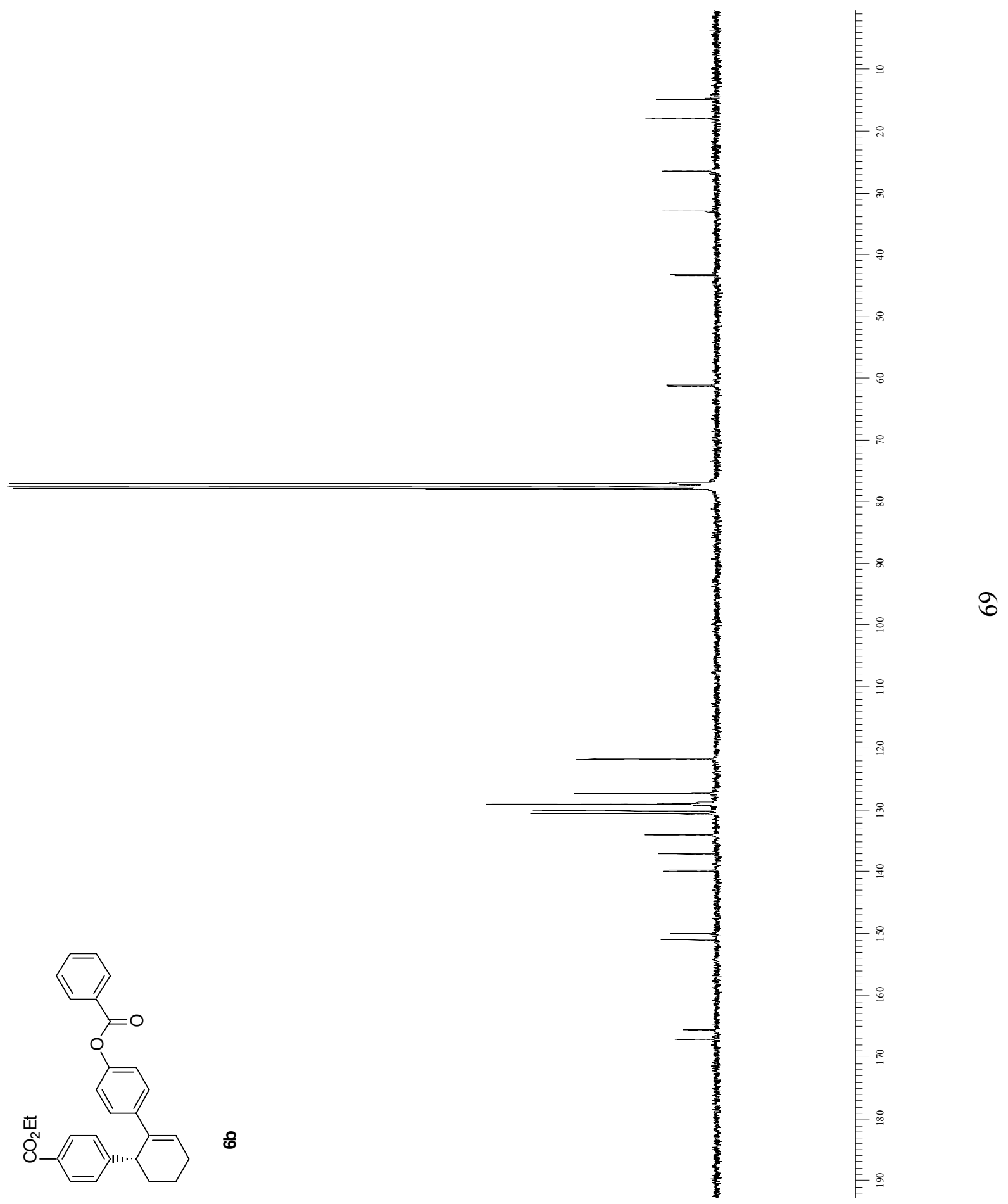


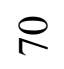

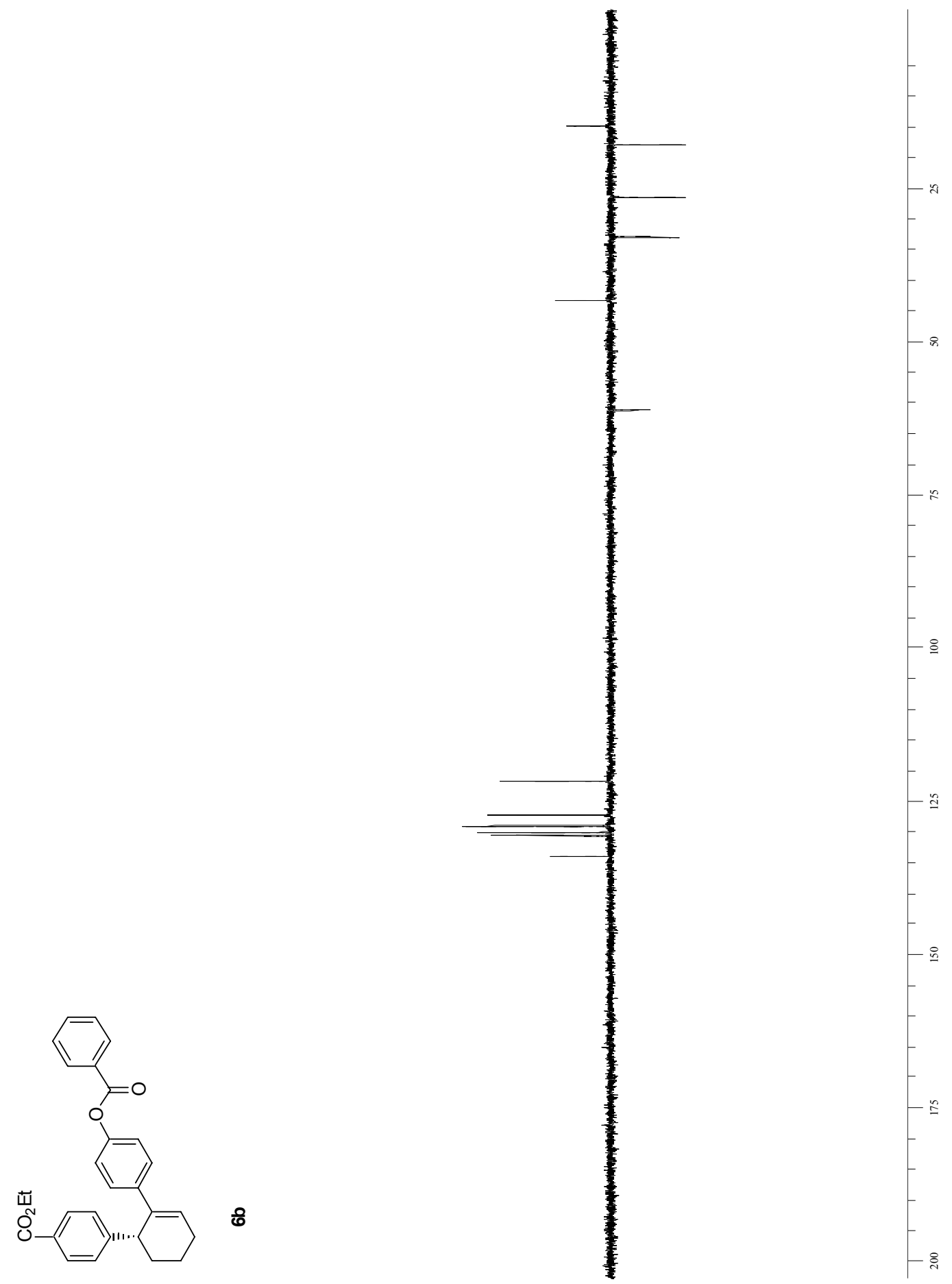




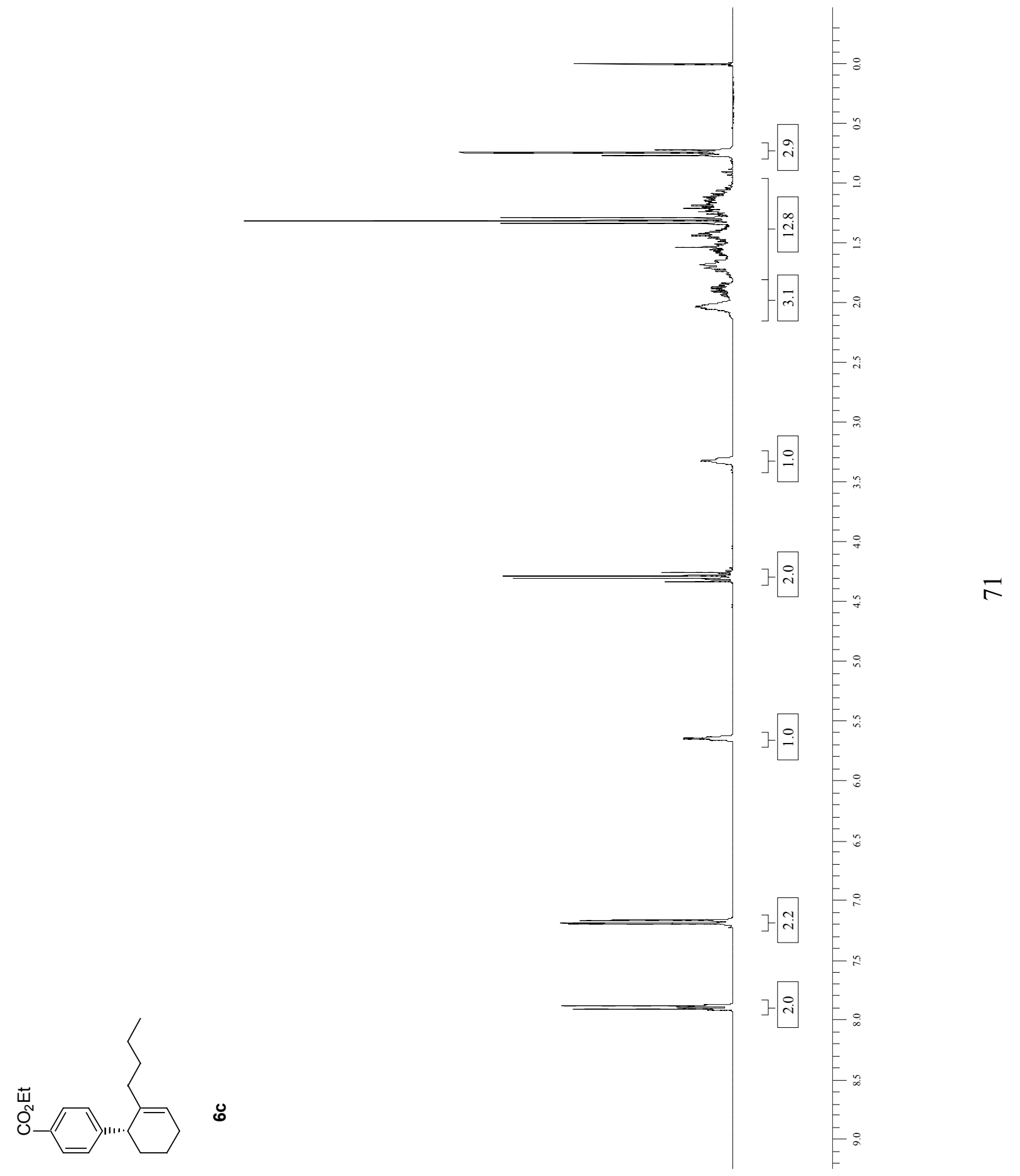




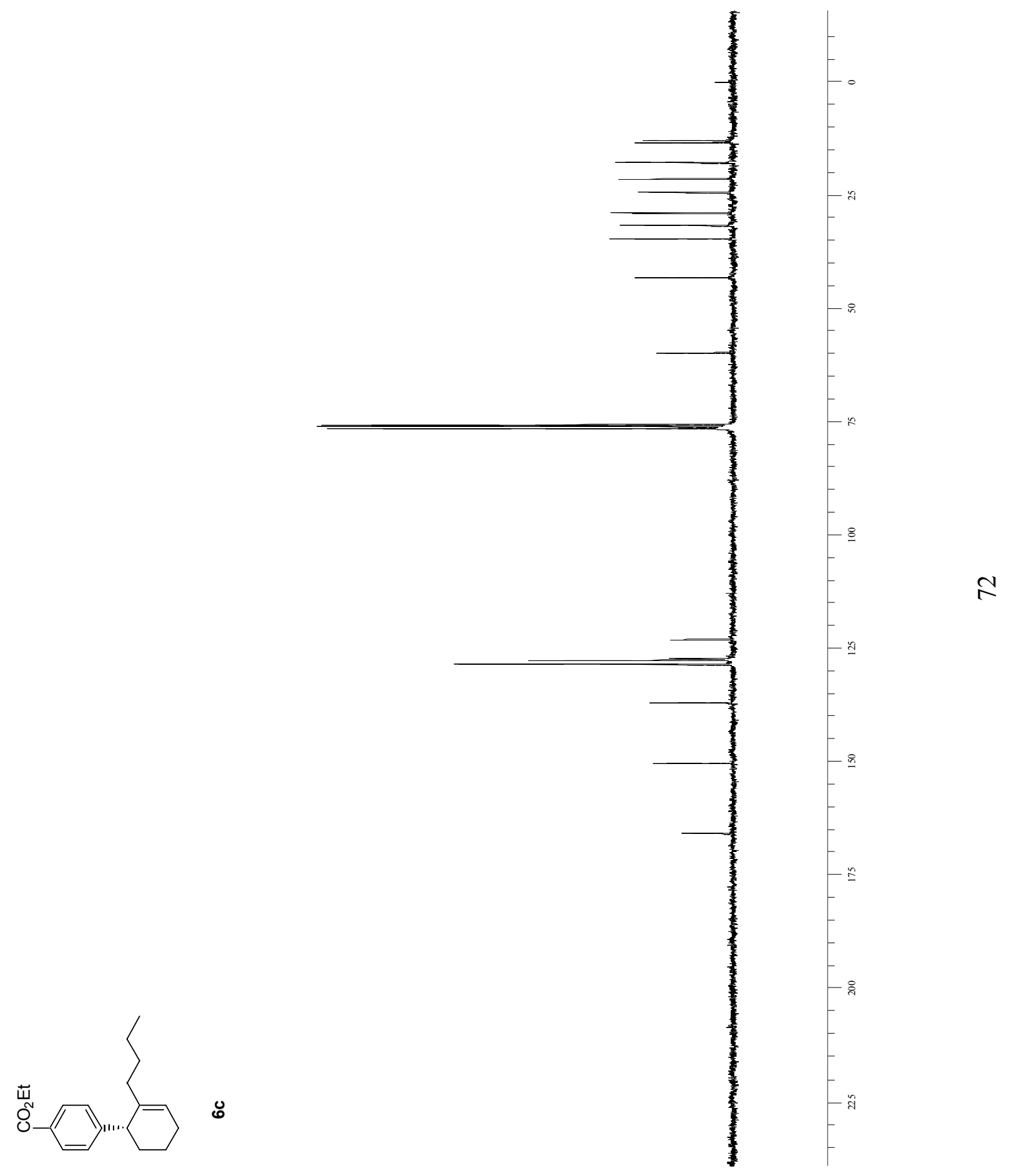




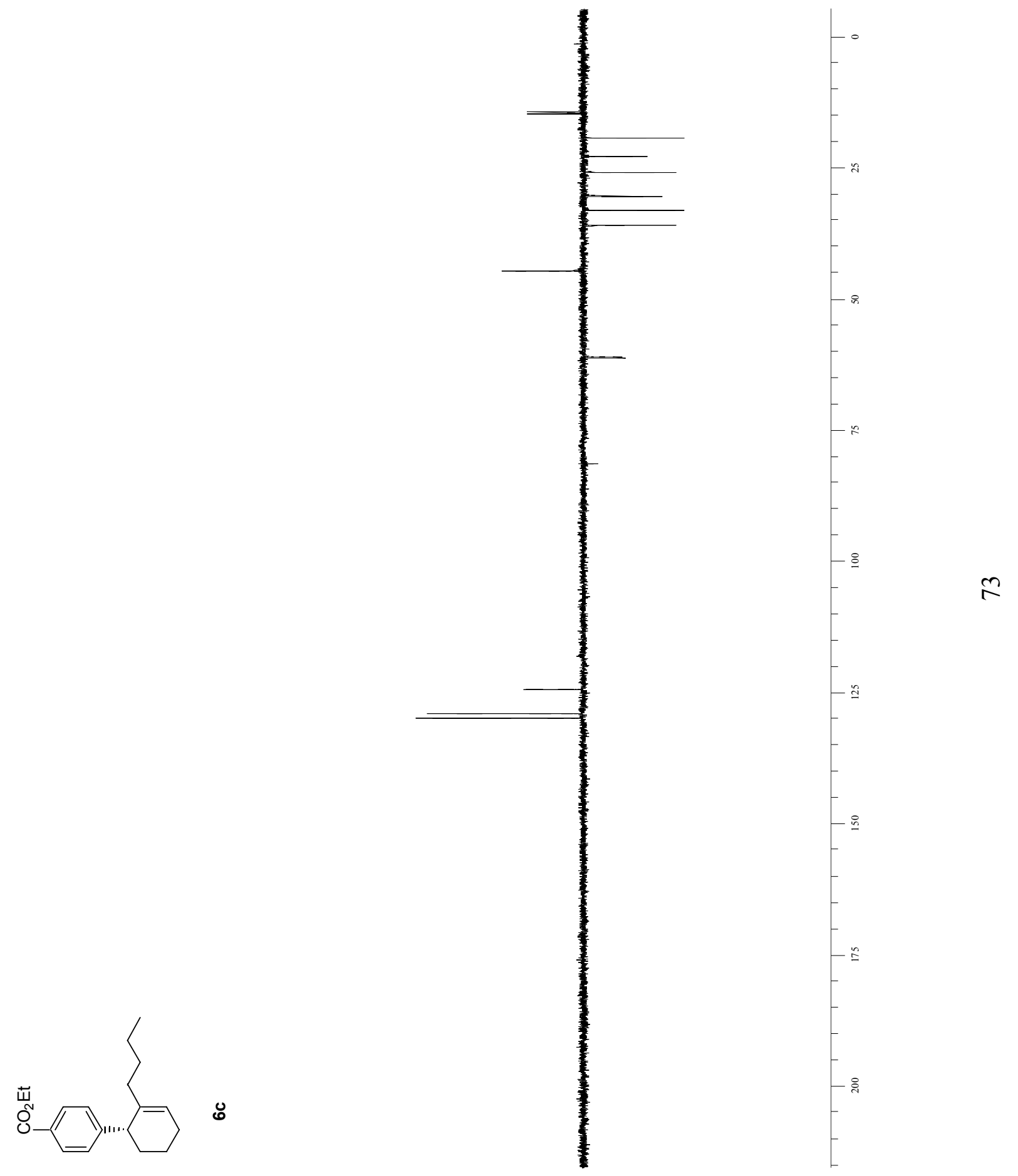




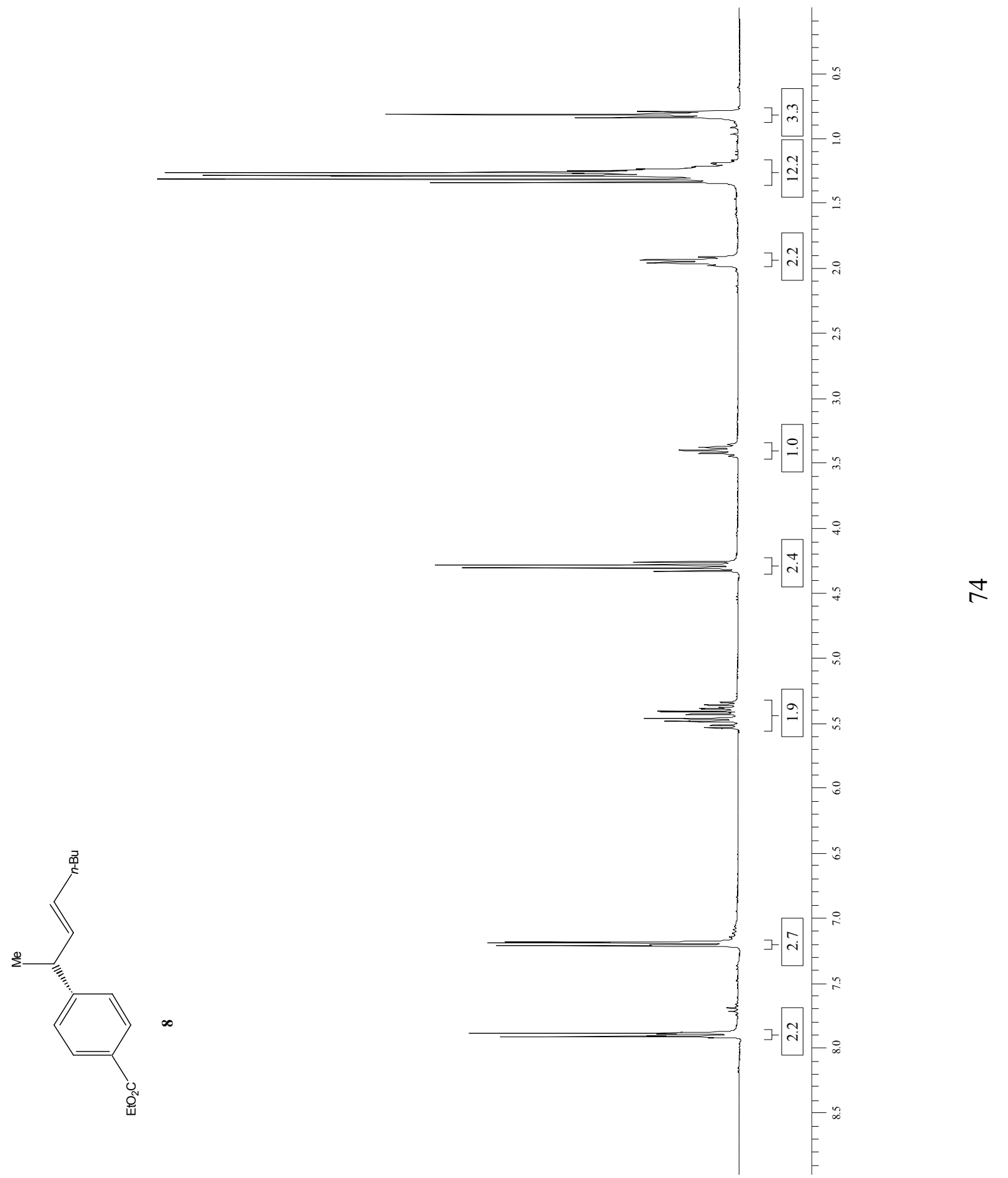




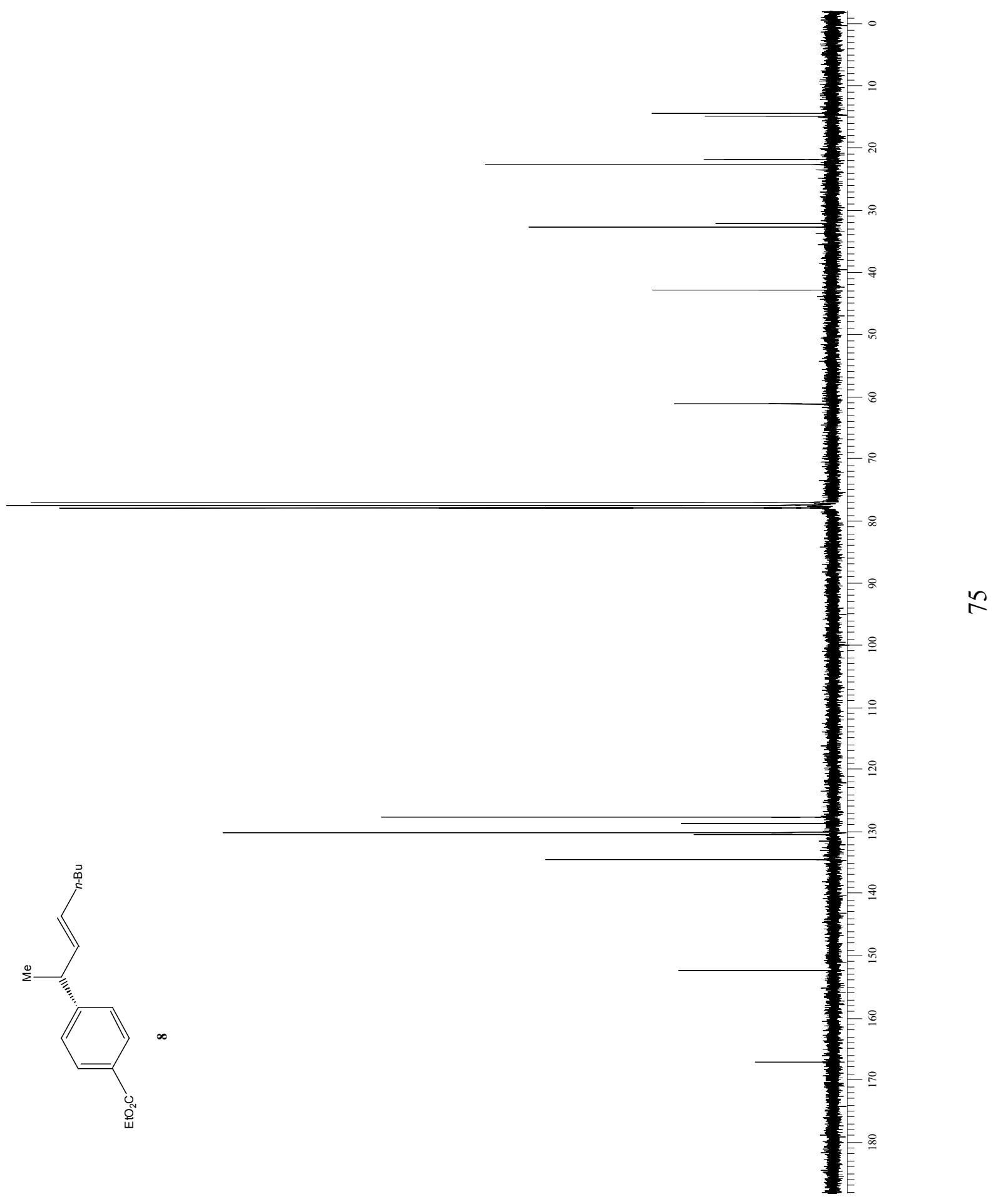


2
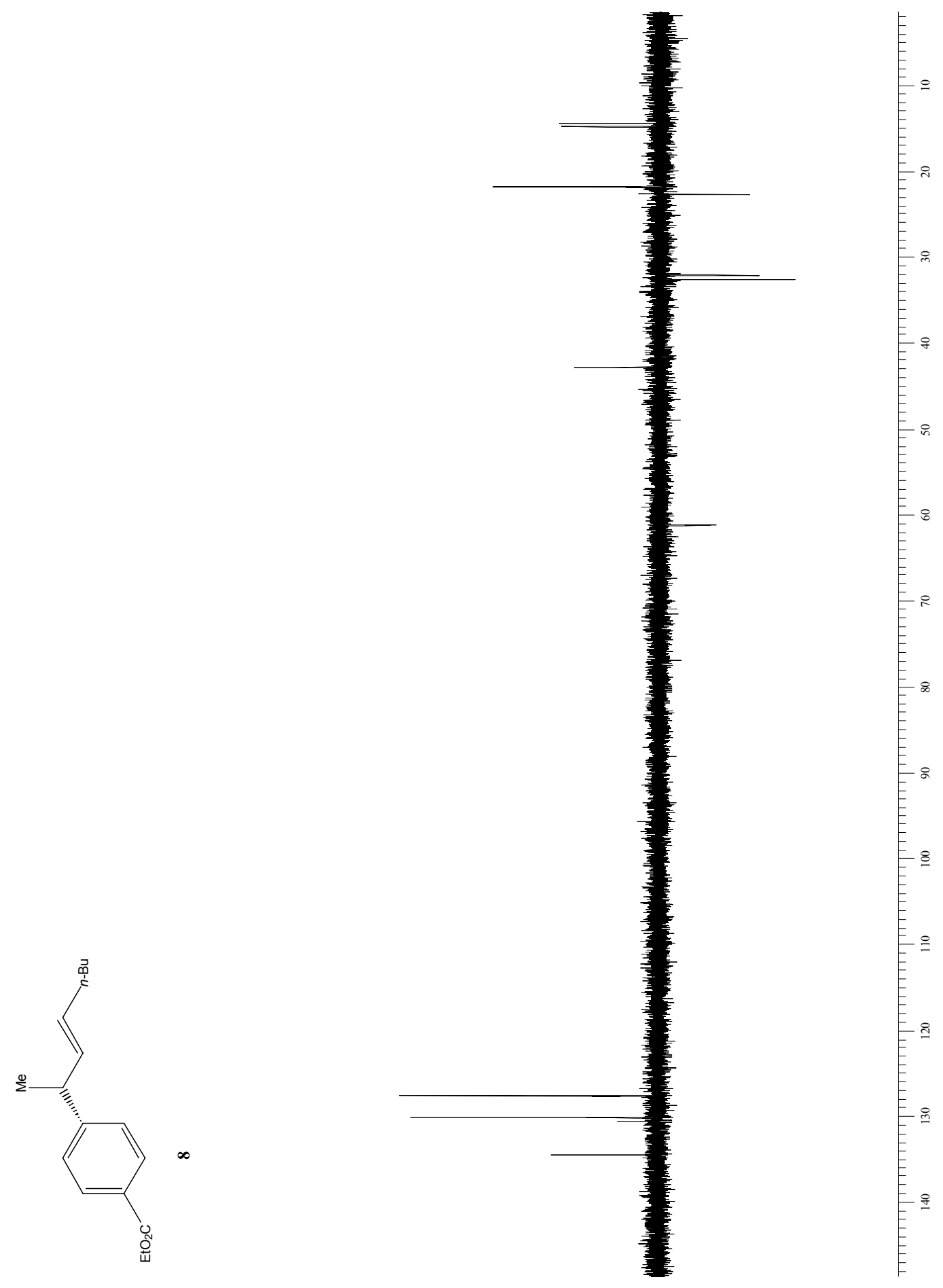

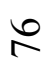

